\title{
Rounding corners of polygons and the embedded contact homology of $T^{3}$
}

\author{
Michael Hutchings \\ MichaEl SUllivaN
}

\begin{abstract}
The embedded contact homology (ECH) of a 3-manifold with a contact form is a variant of Eliashberg-Givental-Hofer's symplectic field theory, which counts certain embedded $J$-holomorphic curves in the symplectization. We show that the ECH of $T^{3}$ is computed by a combinatorial chain complex which is generated by labeled convex polygons in the plane with vertices at lattice points, and whose differential involves "rounding corners". We compute the homology of this combinatorial chain complex. The answer agrees with the Ozsváth-Szabó Floer homology $H F^{+}\left(T^{3}\right)$.
\end{abstract}

57R58; 57M27

\section{Introduction}

\subsection{Motivation}

Let $Y$ be a closed oriented 3-manifold with a contact form, ie a $1-$ form $\lambda$ such that $\lambda \wedge d \lambda>0$. The corresponding contact structure is the 2 -plane field $\xi:=\operatorname{Ker}(\lambda)$; this is oriented by $d \lambda$. Also associated to $\lambda$ is the Reeb vector field $R$ characterized by $\lambda(R)=1$ and $R\lrcorner d \lambda=0$. A periodic orbit of the Reeb flow $R$ is called a Reeb orbit. We assume that the Reeb orbits are nondegenerate or Morse-Bott. For $\Gamma \in H_{1}(Y)$, one can then define the "embedded contact homology" $E C H_{*}(Y, \lambda ; \Gamma)$, as we explain in Section 11.2. This is the homology of a chain complex which is generated by certain unions of Reeb orbits with total homology class $\Gamma$. The differential counts "maximal index" $J$-holomorphic curves in $\mathbb{R} \times Y$, for a suitable almost complex structure $J$. These maximal index curves turn out to be embedded, except that they may contain multiple covers of "trivial cylinders" $\mathbb{R} \times \gamma$ where $\gamma$ is a Reeb orbit. The embedded contact homology is a relatively $\mathbb{Z} / N$-graded $\mathbb{Z}$-module, where $N$ is the divisibility of the image of $c_{1}(\xi)+2 \operatorname{PD}(\Gamma)$ in $\operatorname{Hom}\left(H_{2}(Y), \mathbb{Z}\right)$. When $\Gamma=0$, there is a canonical absolute $\mathbb{Z} / N$-grading.

Embedded contact homology (ECH) is analogous to the periodic Floer theory (PFH) for mapping tori considered by Hutchings, Sullivan and Thaddeus $[11 ; 9 ; 10]$. ECH is 
similar to the symplectic field theory (SFT) of Eliashberg-Givental-Hofer [7], but has different generators and grading, and counts more restricted $J$-holomorphic curves. Unlike SFT, which is highly sensitive to the contact structure, ECH is conjectured to be a topological invariant, except that it detects the Euler class of the contact structure via the identification (1) below. More precisely, we conjecture that $E C H_{*}(Y, \lambda ; \Gamma)$ agrees with the Seiberg-Witten Floer homology $\widetilde{H M} *(-Y)$ defined by Kronheimer and Mrowka [12] and summarized with Ozsváth and Szabó in [14], or the conjecturally isomorphic Ozsváth-Szabó Floer homology $\mathrm{HF}_{*}^{+}(-Y)$ defined in [18], as follows. The latter two Floer homologies depend on the choice of a spin-c structure $\mathfrak{s}$ on $-Y$, which is equivalent to a spin-c structure on $Y$. Let $\operatorname{Spin}^{c}(Y)$ denote the set of spin-c structures on $Y$. Recall that an oriented 2-plane field on $Y$ determines a spin-c structure, cf Turaev [28]. Hence the contact structure $\xi$ gives rise to an $H^{2}(Y ; \mathbb{Z})$-equivariant bijection

$$
\mathfrak{s}_{\xi}: H_{1}(Y) \stackrel{\simeq}{\longrightarrow} \operatorname{Spin}^{c}(Y)
$$

sending 0 to the spin-c structure determined by the oriented 2-plane field $\xi$.

Conjecture 1.1 Let $Y$ be a closed oriented 3-manifold with a contact form $\lambda$. Then for $\Gamma \in H_{1}(Y)$, the embedded contact homology is related to the Seiberg-Witten and Ozsváth-Szabó Floer homologies by

$$
\widetilde{H M}_{*}\left(-Y, \mathfrak{s}_{\xi}(\Gamma)\right) \simeq E C H_{*}(Y, \lambda ; \Gamma) \simeq H F_{*}^{+}\left(-Y, \mathfrak{s}_{\xi}(\Gamma)\right),
$$

up to a grading shift.

Recall that Taubes's "SW=Gr" theorem [25] states that the Seiberg-Witten invariant of a closed symplectic 4-manifold $X$ is equivalent to the "Gromov invariant", which is a certain count of embedded (except for multiply covered tori) $J$-holomorphic curves in $X$. The conjectural relation between ECH and Seiberg-Witten Floer homology can be regarded as an analogue of "SW=Gr" for the noncompact symplectic manifold $\mathbb{R} \times Y$. This was the original motivation for the definition of PFH and ECH. Note also that the Ozsváth-Szabó Floer homology has been given a four-dimensional reformulation by Lipshitz [15]. It is possible that the latter could be directly related to ECH by defining a more general theory including both as special cases.

A proof of Conjecture 1.1, while perhaps a long way off, would have implications for contact dynamics. For example, one version of the Weinstein conjecture asserts that any contact 1 -form $\lambda$ on a closed oriented 3-manifold $Y$ has a Reeb orbit, see eg Abbas-Cieliebak-Hofer [1]. If $\lambda$ has no Reeb orbit, then by definition, the ECH chain complex has only one generator given by the empty set of Reeb orbits, so

$$
E C H_{*}(Y, \lambda ; \Gamma)= \begin{cases}\mathbb{Z}, & \Gamma=0 \text { and } *=0, \\ 0, & \text { otherwise. }\end{cases}
$$


However, Tom Mrowka has pointed out to us that by results in Kronheimer-Mrowka [12], for any closed oriented 3-manifold $Y$, if $\mathfrak{s}$ is a spin-c structure with $c_{1}(\mathfrak{s})$ torsion then $\widetilde{H M}_{*}(Y, \mathfrak{s})$ is infinitely generated. Since $T Y$ is a trivial bundle, one can always find a spin-c structure $\mathfrak{s}$ with $c_{1}(\mathfrak{s})=0$. Therefore, the first part of Conjecture 1.1 implies the Weinstein conjecture for every closed oriented 3-manifold.

ECH has some additional structure, analogous to structures in the Seiberg-Witten and Ozsváth-Szabó Floer homologies. For example, there is a canonical element

$$
c(\lambda) \in E C H_{0}(Y, \lambda ; 0) \text {. }
$$

In the ECH chain complex, the homology class $c(\lambda)$ is represented by the empty set of Reeb orbits. Under the conjectured isomorphisms (2), $c(\lambda)$ may agree with the Ozsváth-Szabó contact invariant [19], and the Seiberg-Witten analogue implicit in the paper by Kronheimer and Mrowka [13].

Further motivation for studying ECH (and PFH) is that it is expected to be the recipient of (yet to be defined) relative Gromov invariants of symplectic 4-manifolds with boundary. For example, Taubes has proposed $[24 ; 26]$ that the Gromov invariant may be extended to near-symplectic 4-manifolds by counting $J$-holomorphic curves in the complement of the circles where the near-symplectic form vanishes. We expect such a counting invariant to take values in the embedded contact homology of a disjoint union of $S^{1} \times S^{2}$ 's, one for each vanishing circle, with the contact form studied by Taubes in [27]. Also, the relative Gromov invariants should enter into gluing formulas for Gromov invariants of closed symplectic 4-manifolds cut along 3-manifolds.

Much of the embedded contact homology story is still conjectural. In particular, a proof that $E C H_{*}(Y, \lambda ; \Gamma)$ is well-defined is currently in preparation; the precise statement is given here as Conjecture 11.10. In any case, the results in this paper from Section 2 to Section 10, while motivated by this conjecture, are logically independent of it.

\subsection{The embedded contact homology of $T^{3}$}

This paper is concerned with computations of embedded contact homology. We will restrict attention to the example of $Y=T^{3}$, although the methods developed here are applicable to some other simple contact manifolds such as $S^{1} \times S^{2}$, or $T^{2}$-bundles over $S^{1}$, see Section 12.2. For each positive integer $n$, there is a standard contact form $\lambda_{n}$ on $T^{3}$ defined as follows. We choose the following coordinates on $T^{3}$ that depend on $n$ :

$$
T^{3}=S^{1} \times T^{2}=(\mathbb{R} / 2 \pi n \mathbb{Z})_{\theta} \times\left(\mathbb{R}^{2} / \mathbb{Z}^{2}\right)_{x, y}
$$


Then

$$
\lambda_{n}:=\cos \theta d x+\sin \theta d y .
$$

The associated Reeb vector field is given by

$$
R=\cos \theta \partial_{x}+\sin \theta \partial_{y} .
$$

In particular, $\lambda_{n}$ is a Morse-Bott contact form; for each $\theta \in \mathbb{R} / 2 \pi n \mathbb{Z}$ with $\tan \theta \in$ $\mathbb{Q} \cup\{\infty\}$, there is an $S^{1}$-family of Reeb orbits in $\{\theta\} \times T^{2}$.

It turns out that to compute $\mathrm{ECH}$ in this example, for suitable almost complex structures $J$, the relevant $J$-holomorphic curves can be counted quite explicitly. For this purpose we modify some arguments from our previous paper [10] on the PFH of a Dehn twist, and use some results of Taubes [27]. Consequently, for

$$
\Gamma \in \mathbb{Z}^{2}=H_{1}\left(T^{2}\right) \subset H_{1}\left(S^{1} \times T^{2}\right),
$$

we can define a combinatorial chain complex $\bar{C}_{*}(2 \pi n ; \Gamma)$, see Section 1.3 and Section 3 , whose homology $\bar{H}_{*}(2 \pi n ; \Gamma)$ agrees with the embedded contact homology of $T^{3}$. (Throughout this paper we adopt the convention that changing the letter ' $C$ ' to ' $H$ ', indicates passing from a chain complex to its homology.) Namely:

Theorem 1.2 If Conjecture 11.10 holds (so that ECH is well-defined), then

$$
E C H_{*}\left(T^{3}, \lambda_{n} ; \Gamma\right) \simeq \bar{H}_{*}(2 \pi n ; \Gamma) .
$$

Note that since all Reeb orbits have homology classes in the subgroup $H_{1}\left(T^{2}\right)$, the ECH automatically vanishes for $\Gamma \in H_{1}\left(S^{1} \times T^{2}\right) \backslash H_{1}\left(T^{2}\right)$, because the chain complex has no generators.

As will be explained in Section 12.1, ECH has some variants and additional structure. In particular, there is a degree -2 operation

$$
U: E C H_{*}(Y, \lambda ; \Gamma) \longrightarrow E C H_{*-2}(Y, \lambda ; \Gamma),
$$

which counts $J$-holomorphic curves with a marked point mapping to a chosen point in $\mathbb{R} \times Y$. In the case of $T^{3}$, the operation $U$ corresponds to a combinatorial chain map

$$
U: \bar{C}_{*}(2 \pi n ; \Gamma) \longrightarrow \bar{C}_{*-2}(2 \pi n ; \Gamma)
$$

defined in Section 4. We can now state our main computational result:

Theorem 1.3 For every positive integer $n$ :

(a) If $\Gamma \neq 0$, then $\bar{H}_{*}(2 \pi n ; \Gamma)=0$. 
(b) For $\Gamma=0$,

$$
\bar{H}_{i}(2 \pi n ; 0) \simeq\left\{\begin{array}{cc}
\mathbb{Z}^{3}, & i \geq 0, \\
0, & i<0 .
\end{array}\right.
$$

(c) For all $i \geq 2$, the map $U$ induces an isomorphism

$$
U: \bar{H}_{i}(2 \pi n ; 0) \stackrel{\simeq}{\longrightarrow} \bar{H}_{i-2}(2 \pi n ; 0) .
$$

In particular, the ECH of $\left(T^{3}, \lambda_{n}\right)$ does not depend on $n$. By contrast, the simplest version of SFT, namely cylindrical contact homology, distinguishes the contact structures $\xi_{n}=\operatorname{Ker}\left(\lambda_{n}\right)$; see Eliashberg-Givental-Hofer [7, Theorem 1.9.9], and for generalizations see Bourgeois-Colin [3]. On the other hand, the contact invariant (3) for $\lambda_{n}$ does depend on $n$, see Section 12.1.2.

The above computation of the ECH of $T^{3}$, together with some additional structure on it described in Section 12.1.3, agree perfectly with $H F^{+}\left(T^{3}\right)$ as computed in Ozsváth-Szabó [17], and also $\widetilde{H M} *\left(T^{3}\right)$ as computed in Kronheimer-Mrowka [12]. This provides a nontrivial check of Conjecture 1.1.

\subsection{Rounding corners of polygons}

We now introduce the combinatorial chain complex $\bar{C}_{*}(2 \pi n ; \Gamma)$, along with two variants $\widetilde{C}_{*}(2 \pi n ; \Gamma)$ and $C_{*}(2 \pi n ; \Gamma)$, in the simplest case where $n=1$ and $\Gamma=0$.

1.3.1 The generators The complex $C_{*}=C_{*}(2 \pi ; 0)$ is a free $\mathbb{Z}$-module. A generator of $C_{*}$ is a convex polygon in $\mathbb{R}^{2}$, possibly a 2 -gon or a point, such that the corners are lattice points, and every edge is labeled either ' $e$ ' or ' $h$ '. To fix the signs in the differential, we choose an ordering of the ' $h$ ' edges, and we declare that a reordering of the ' $h$ ' edges multiplies the generator by the sign of the reordering permutation.

1.3.2 The grading The grading, or index, of a generator $\alpha$ is defined by

$$
I(\alpha):=2(\# L(\alpha)-1)-\# h(\alpha) .
$$

Here $\# L(\alpha)$ denotes the cardinality of the set $L(\alpha)$ of lattice points on the polygon or enclosed by it, and $\# h(\alpha)$ denotes the number of ' $h$ ' edges. By Pick's formula for the area of a lattice polygon, equation (6) is equivalent to

$$
I(\alpha)=2 \operatorname{Area}(\alpha)+\# \ell(\alpha)-\# h(\alpha) .
$$

Here $\operatorname{Area}(\alpha)$ denotes the area enclosed by $\alpha$, and $\ell(\alpha)$ denotes the sum of the divisibilities of the edges of the polygon.

For example, equation (7) implies that $I(\alpha) \geq 0$, and the only index zero generators are the following: 
- Points, which we denote by $p(u)$ where $u \in \mathbb{Z}^{2}$.

- 2-gons with vertices $u, v \in \mathbb{Z}^{2}$ with $u-v$ indivisible and with both edges labeled ' $h$ '. If the edge from $u$ to $v$ is first in the ordering, then we denote this generator by $h(u, v)$. Thus $h(v, u)=-h(u, v)$.

1.3.3 The differential The differential $\delta: C_{*} \rightarrow C_{*-1}$ is defined as follows. Roughly, if $\alpha$ is a generator, then $\delta \alpha$ is the signed sum of all generators $\beta$ obtained by "rounding a corner" and "locally losing one ' $h$ "'. More precisely:

- "Rounding a corner" means that $L(\beta)=L(\alpha) \backslash c$ where $c$ is a corner of $\alpha$.

- "Locally losing one ' $h$ "' means the following. First, at least one of the two edges in $\alpha$ adjacent to $c$ must be labeled ' $h$ '. Second, of the edges in $\beta$ that are created or shortened by rounding the corner $c$, all are labeled ' $e$ ', except for one when both edges adjacent to $c$ are labeled ' $h$ '. Finally, all other edges in $\beta$ have the same labels as the corresponding edges in $\alpha$.

- To determine the sign, let $\theta$ denote (one of) the ' $h$ ' edge(s) of $\alpha$ adjacent to $c$. Without loss of generality, $\theta$ is last in the ordering of the ' $h$ ' edges of $\alpha$, while the remaining ' $h$ ' edges of $\alpha$ are ordered the same way as the ' $h$ ' edges of $\beta$ under the obvious bijection between them. Then the differential coefficient $\langle\delta \alpha, \beta\rangle=+1$ if $\theta$ comes immediately after $c$ as we traverse $\alpha$ counterclockwise. If $\theta$ comes immediately before $c$, then $\langle\delta \alpha, \beta\rangle=-1$.

Here is a random example of $\delta$. In the pictures below, the unmarked edges are labeled ' $e$ ', and on the left side the bottom ' $h$ ' edge is first in the ordering.

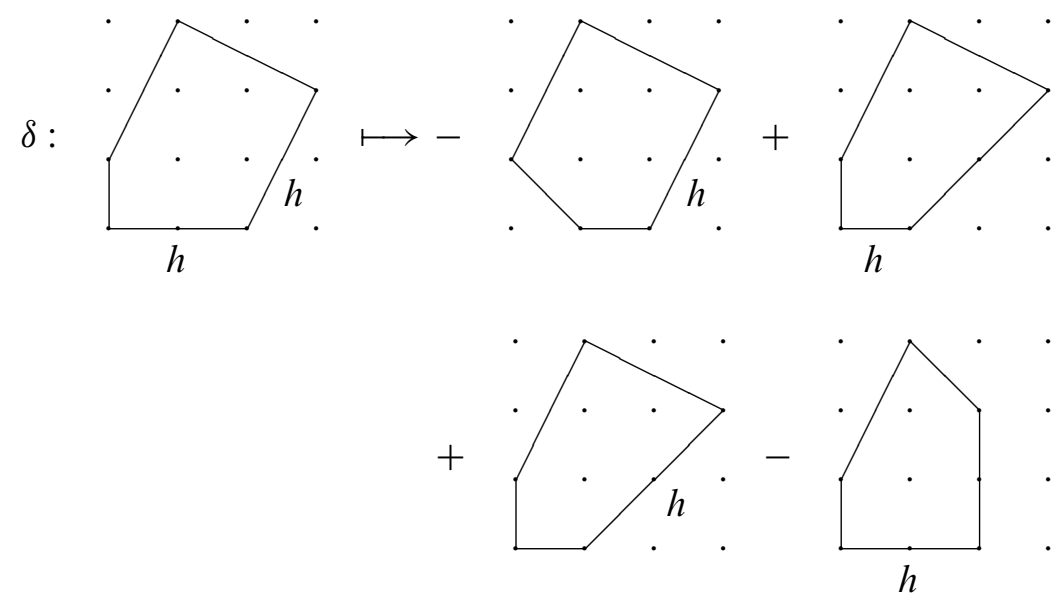

It is shown in Section 3.2 that $\delta$ has degree -1 and $\delta^{2}=0$. 
1.3.4 The homology To further illustrate the definitions, let us compute the degree-0 homology $H_{0}$. The index one generators are the following:

- 2-gons with no lattice points in the interiors of the edges and one edge labeled ' $e$ ' and one edge labeled ' $h$ '. We denote such a generator by $e(u, v)$, where $u$ and $v$ are the two corners and the edge from $u$ to $v$ is labeled ' $e$ '.

- Triangles enclosing no lattice points except the corners $u, v, w$, with all three edges labeled ' $h$ '. We denote such a generator by $h(u, v, w)$, where $u, v, w$ are listed in counterclockwise order and the edges are ordered counterclockwise.

It follows from the definitions that

$$
\begin{gathered}
\delta p(u)=\delta h(u, v)=0 \\
\delta e(u, v)=p(u)-p(v), \\
\delta h(u, v, w)=h(u, v)+h(v, w)+h(w, u) .
\end{gathered}
$$

Therefore $H_{0}$ is generated by the homology classes $[p(u)]$ and $[h(u, v)]$ modulo the relations

$$
\begin{aligned}
{[p(u)] } & =[p(v)], \\
{[h(u, v)]+[h(v, w)] } & =[h(u, w)] .
\end{aligned}
$$

The computation of the higher homology is more complicated, but the result is simpler. Let $\Lambda$ be a convex polygon that encloses and contains $k$ lattice points with $k \geq 2$. Let $E_{\Lambda}$ denote the generator consisting of the polygon $\Lambda$ with all edges labeled ' $e$ '. Let $H_{\Lambda}$ denote the sum of all generators consisting of the polygon $\Lambda$ with one edge labeled ' $h$ ' and all other edges labeled ' $e$ '. It follows from the definitions that $E_{\Lambda}$ and $H_{\Lambda}$ are cycles. We will see in Section 5 that the homology classes of these cycles depend only on $k$. Moreover,

$$
H_{2 k-2}=\mathbb{Z}\left\{E_{\Lambda}\right\}, \quad H_{2 k-3}=\mathbb{Z}\left\{H_{\Lambda}\right\} .
$$

1.3.5 Variants Let $\widetilde{C}_{*}$ denote the chain complex $C_{*}$ regarded as a module over the group ring $\mathbb{Z}\left[\mathbb{Z}^{2}\right]$, where $\mathbb{Z}^{2}$ acts on the generators by translation in the plane. The homology $\widetilde{H}_{*}$ can be read off from the preceding calculations. Let $\mathcal{I}\left(\mathbb{Z}^{2}\right)$ denote the augmentation ideal in $\mathbb{Z}\left[\mathbb{Z}^{2}\right]$, ie the kernel of the augmentation map $\mathbb{Z}\left[\mathbb{Z}^{2}\right] \rightarrow \mathbb{Z}$ sending a group ring element to the sum of its coefficents. Also, let $\mathbb{Z}$ denote the $\mathbb{Z}\left[\mathbb{Z}^{2}\right]$-module with one generator on which $\mathbb{Z}^{2}$ acts by the identity, ie

$$
\underline{\mathbb{Z}}:=\{a \mid(x-1) a=(y-1) a=0\}
$$


where $x$ and $y$ denote generators of $\mathbb{Z}^{2}$. Then we have

$$
\widetilde{H}_{*} \simeq\left\{\begin{array}{cl}
0, & *<0, \\
\mathcal{I}\left(\mathbb{Z}^{2}\right) \oplus \mathbb{Z}, & *=0, \\
\mathbb{Z}, & *>0
\end{array}\right.
$$

Here the isomorphism $\widetilde{H}_{0} \simeq \mathcal{I}\left(\mathbb{Z}^{2}\right) \oplus \mathbb{Z}$ sends $[p(u)]$ to the generator of $\mathbb{Z}$ and $[h(u, v)]$ to $u-v \in \mathcal{I}\left(\mathbb{Z}^{2}\right)$.

We can obtain another complex $\bar{C}_{*}$ over $\mathbb{Z}$ by declaring generators to be equivalent when they differ by translation in the plane. The homology $\bar{H}_{*}$ of $\bar{C}_{*}$ is partially but not entirely determined by $\widetilde{H}_{*}$, via a "universal coefficient spectral sequence"

$$
E_{p, q}^{2}=\operatorname{Tor}_{p}\left(\widetilde{H}_{q}, \mathbb{Z}\right) \Longrightarrow \bar{H}_{p+q} .
$$

It turns out that some of the differentials in the spectral sequence (8) are nonzero, and we will find in Section 8 that

$$
\bar{H}_{*} \simeq\left\{\begin{array}{cc}
0, & *<0 \\
\mathbb{Z}^{3}, & * \geq 0
\end{array}\right.
$$

1.3.6 Geometric interpretation The rough idea of the relation between $\bar{H}_{*}$ and $E C H_{*}\left(T^{3}, \lambda_{1} ; 0\right)$ is as follows. For a generator of $\bar{C}_{*}$, each edge of the polygon corresponds to a circle of Reeb orbits in $T^{3}$. The labels ' $e$ ' and ' $h$ ' reflect the fact that each circle of Reeb orbits for the Morse-Bott contact form $\lambda_{1}$ can be perturbed into two nondegenerate orbits, one elliptic and one hyperbolic. It turns out that in $\mathbb{R} \times T^{3}$, every $J$-holomorphic curve counted by the $\mathrm{ECH}$ differential consists of one embedded genus zero component with two positive ends (corresponding to the edges adjacent to the corner being rounded) and an arbitrary number of negative ends (corresponding to the edges created by rounding), together with some $\mathbb{R}$-invariant cylinder components (corresponding to the edges or parts thereof not involved in the corner rounding). The chain complex $\widetilde{C}_{*}$ corresponds to a "partially twisted" version of $\mathrm{ECH}$ in which one keeps track of some information about the relative homology classes of the $J$-holomorphic curves.

1.3.7 The rest of the paper The combinatorial chain complexes for general $n$ and $\Gamma$, roughly speaking, involve left-turning polygonal paths of rotation number $n$ and period $\Gamma$. The precise definitions require some care and are given in Section 3, after some combinatorial preliminaries in Section 2. The combinatorial chain map $U$ is defined in Section 4; aside from its significance for $\mathrm{ECH}$, it will help compute the homology of the combinatorial chain complexes. In Section 5-Section 8 we compute 
the homology of all of the above combinatorial chain complexes and in particular prove Theorem 1.3.

In Section 9 we establish an axiomatic characterization of the combinatorial chain complexes. In Section 10 we recall and prove some relevant facts about $J$-holomorphic curves in $\mathbb{R} \times T^{3}$. In Section 11 we outline the definition of ECH and prove Theorem 1.2. The proof uses the results in Section 10 to show that the chain complex computing the ECH of $T^{3}$ satisfies the axioms in Section 9. In Section 12 we make some concluding remarks.

1.3.8 Acknowledgments We thank Y Eliashberg, $\mathrm{P}$ Kronheimer, $\mathrm{T}$ Mrowka, $\mathrm{P}$ Ozsváth, Z Szabó, and C Taubes for enlightening discussions. We thank the anonymous referee for many helpful comments. The first author was partially supported by NSF grant DMS-0204681 and the Alfred P Sloan Foundation. The second author was partially supported by NSF grant DMS- 0305825 .

\subsubsection{Index of frequently used notation}

\begin{tabular}{|c|c|}
\hline $\mathbb{Z}$ & $\begin{array}{l}\text { the } \mathbb{Z}\left[\mathbb{Z}^{2}\right] \text {-module with one generator on which } \\
\mathbb{Z}^{2} \text { acts trivially, see Section } 1.3 .5\end{array}$ \\
\hline $\mathcal{I}\left(\mathbb{Z}^{2}\right)$ & augmentation ideal, see Section 1.3.5 and also Section 6.2 \\
\hline$\Lambda \backslash c$ & rounding of $\Lambda$ at $c$, see Section 2.2 \\
\hline$\Lambda^{\prime} \leq \Lambda$ & $\Lambda^{\prime}$ is to the left of $\Lambda$, see Section 2.3 \\
\hline $\mathcal{A}$ & $\begin{array}{l}\text { length of a polygonal path defined in equation (14), and } \\
\text { symplectic action of an orbit set defined in Definition } 10.6\end{array}$ \\
\hline$C_{*}(2 \pi n ; \Gamma)$ & $\begin{array}{l}\text { combinatorial chain complex generated by polygons } \\
\text { of rotation number } n \text { and period } \Gamma \text {, see Section } 3.1\end{array}$ \\
\hline$\widetilde{C}_{*}(2 \pi n ; \Gamma)$ & above complex regarded as a $\mathbb{Z}\left[\mathbb{Z}^{2}\right]$-module \\
\hline $\bar{C}_{*}(2 \pi n ; \Gamma)$ & above complex modulo translation of polygons \\
\hline$C_{*}(\Lambda)$ & complex of polygons to the left of $\Lambda$, see Section 3.1.5 \\
\hline$I$ & $\begin{array}{l}\text { combinatorial relative index defined in Section } 3.1 \text {, and } \\
\text { analytical relative index defined in Section } 11.1\end{array}$ \\
\hline$\delta$ & combinatorial differential defined in Section 3.1 \\
\hline$E_{\Lambda}, H_{\Lambda}$ & two distinguished cycles defined in Section 3.1.6 \\
\hline$c_{\theta}$ & corner at $\theta$, see Section 4 \\
\hline$U$ & $\begin{array}{l}\text { combinatorial degree }-2 \text { chain map defined in Section } 4 \text {, and } \\
\text { analytical degree }-2 \text { map defined in Section } 12.1 .4\end{array}$ \\
\hline$C^{(j)}$ & subcomplex where $I-\# h=j$, see Definition 5.1 \\
\hline$\partial_{c}$ & connecting homomorphism in long exact sequence in Section 5.1 \\
\hline$F_{\theta}$ & flattening chain map, see Definition 6.13 \\
\hline
\end{tabular}




\begin{tabular}{|c|c|}
\hline$Z_{n}(a, b)$ & a degree zero cycle defined in Section 6.2 \\
\hline$C X_{*}$ & subcomplex consisting of $x$-axis polygons, see Section 7.1 \\
\hline$S$ & splicing chain map, see Section 7.1 \\
\hline$H_{2}(Y, \alpha, \beta)$ & relative homology classes as in Definition 10.2 \\
\hline $\mathcal{M}^{J}$ & moduli space of $J$-holomorphic curves, see Section 10 \\
\hline ind & SFT index, see Definition 11.1 \\
\hline$E C H_{*}(Y, \lambda ; \Gamma)$ & embedded contact homology, see Section 11.2 \\
\hline$\widetilde{E C H}_{*}(Y, \lambda ; \Gamma, G)$ & twisted embedded contact homology, see Section 11.2 \\
\hline$\partial$ & embedded contact homology differential, see Section 11.2 \\
\hline
\end{tabular}

\section{Rounding corners of polygonal paths}

In this section we lay the foundations for our combinatorial investigations. We are preparing to define (in Section 3) a general combinatorial chain complex $C_{*}(2 \pi n ; \Gamma)$ in which the generating polygons have rotation number $n \geq 1$, and when $\Gamma \neq 0$ are periodic with period $\Gamma$ rather than closed. In Section 2.1 we define the relevant classes of polygonal paths, which we call "admissible paths". In Section 2.2 we define the corner rounding operation for these polygonal paths. In Section 2.3 we introduce a closely related partial order on admissible paths, and we prove various facts about corner rounding and the partial order which will be needed later. A dictionary between some of this combinatorics and the geometry of pseudoholomorphic curves in $\mathbb{R} \times T^{3}$ will be given later in Section 10.

\subsection{Admissible (left-turning polygonal) paths}

We now define three types of "admissible paths": "open", "closed", and "periodic". Closed and periodic admissible paths will be used to define the chain complex

$$
C_{*}(2 \pi n ; \Gamma)
$$

for $\Gamma=0$ and $\Gamma \neq 0$ respectively, while we will use open admissible paths to help compute its homology.

Let $\Theta$ denote the set of $\theta \in \mathbb{R}$ such that $\tan \theta \in \mathbb{Q} \cup\{\infty\}$. For each $\theta \in \Theta$, there is a unique integer vector

$$
\left(\begin{array}{l}
x_{\theta} \\
y_{\theta}
\end{array}\right) \in \mathbb{Z}^{2}
$$

such that $x_{\theta}, y_{\theta}$ are relatively prime and such that $(\cos \theta, \sin \theta)$ is a positive real multiple of $\left(x_{\theta}, y_{\theta}\right)$. 
Definition 2.1 An open admissible path defined on an interval $I \subset \mathbb{R}$ is a locally constant map $\Lambda: I \backslash T \rightarrow \mathbb{Z}^{2}$, where $T \subset \Theta \cap \operatorname{int}(I)$ is a finite set of edges, and there is a multiplicity function $m: T \rightarrow \mathbb{Z}_{>0}$ such that $\Lambda$ satisfies the "jumping condition"

$$
\frac{d}{d t} \Lambda(t)=\sum_{\theta \in T} m(\theta)\left(\begin{array}{l}
x_{\theta} \\
y_{\theta}
\end{array}\right) \delta_{\theta}(t)
$$

Here $\delta_{\theta}$ denotes the delta function supported at $\theta$. A corner of $\Lambda$ is a component of $I \backslash T$, other than the first and last components. The endpoints of $\Lambda$ are its values on the first and last components of $I \backslash T$. A kink of $\Lambda$ is a corner between consecutive edges $\theta_{1}, \theta_{2}$ with $\theta_{2}-\theta_{1}>\pi$.

That is, $\Lambda$ is a polygonal path in the plane with corners in $\mathbb{Z}^{2}$, parametrized so that it is usually stopped at a corner, and jumps discontinuously to the next corner at time $t$ when the vector $(\cos t, \sin t)$ points in the direction of the corresponding edge. That is, a smooth locally convex curve is naturally parametrized by its tangent direction, and we are extending this notion to polygonal paths. We will sometimes abuse notation and pretend that $\Lambda$ is a continuous, piecewise linear path, moving along straight line segments from one vertex to the next. The path $\Lambda$ turns to the left at its corners, except at kinks where it rotates by more than $\pi$ and hence may turn in any direction. Note that by equation (9), $\Lambda$ determines $m$, while $m$ determines $\Lambda$ up to a $\mathbb{Z}^{2}$ translation ambiguity.

Now fix a positive integer $n$ and an integer vector $\Gamma \in \mathbb{Z}^{2}$. Let $p: \mathbb{R} \rightarrow \mathbb{R} / 2 \pi n \mathbb{Z}$ denote the projection.

Definition 2.2 A periodic admissible path of rotation number $n$ and period $\Gamma$ is a locally constant map $\Lambda: \mathbb{R} \backslash p^{-1}(T) \rightarrow \mathbb{Z}^{2}$, where $T \subset p(\Theta)$ is a finite set of edges, and there is a multiplicity function $m: T \rightarrow \mathbb{Z}_{>0}$ such that $\Lambda$ satisfies the jumping condition

$$
\frac{d}{d t} \Lambda(t)=\sum_{\theta \in p^{-1}(T)} m(p(\theta))\left(\begin{array}{l}
x_{\theta} \\
y_{\theta}
\end{array}\right) \delta_{\theta}(t) .
$$

We also assume that $\Lambda$ satisfies the periodicity condition

$$
\Lambda(t+2 \pi n)=\Lambda(t)+\Gamma,
$$

which by (10) is equivalent to

$$
\sum_{\theta \in T} m(\theta)\left(\begin{array}{l}
x_{\theta} \\
y_{\theta}
\end{array}\right)=\Gamma .
$$


A corner of $\Lambda$ is a component of $(\mathbb{R} / 2 \pi n \mathbb{Z}) \backslash T$. A kink is a corner of length greater than $\pi$. If $\Gamma=0$, we say that $\Lambda$ is a closed admissible path of rotation number $n$.

Example 2.3 In the simplest case of this definition, where $n=1$ and $\Gamma=0$, a closed admissible path $\Lambda$ of rotation number 1 is equivalent to a convex polygonal region $P$ in $\mathbb{R}^{2}$ (possibly a 2 -gon or a 0 -gon) with corners in $\mathbb{Z}^{2}$. The path $\Lambda$ traverses the boundary of $P$ counterclockwise.

Here is an example of a closed admissible path with rotation number $n=2$. The bottom corner is a kink at which the path turns by angle $5 \pi / 4$.

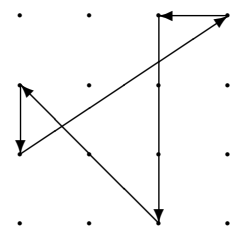

Here is an example of a periodic admissible path with

$$
n=1 \quad \text { and } \quad \Gamma=\left(\begin{array}{l}
2 \\
1
\end{array}\right) .
$$

(The path continues infinitely in both directions.)

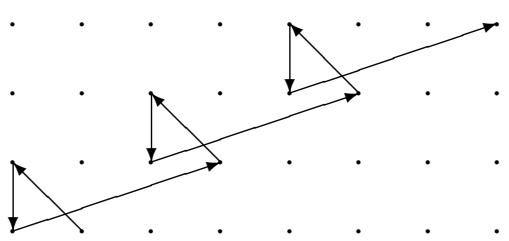

For closed admissible paths with $n>1$, our definition keeps track of the parametrization of the path; that is, $\mathbb{Z} / n$ acts nontrivially on the set of closed admissible paths of rotation number $n$ by precomposing $\Lambda$ with translations on $\mathbb{R}$ by multiples of $2 \pi$. For periodic admissible paths with $\Gamma \neq 0$, precomposing $\Lambda$ with translations by multiples of $2 \pi$ gives a $\mathbb{Z}$ action on the set of periodic admissible paths. (The parametrization is not indicated in the above two pictures.)

We say that two admissible paths $\Lambda, \Lambda^{\prime}$ are of the same type if they are both open and defined on the same interval $I$ and have the same endpoints, or if they are both closed or periodic for the same $n$ and $\Gamma$. 


\subsection{Rounding corners of admissible paths}

We now define the corner rounding operation for admissible paths of any type.

Definition 2.4 Let $\Lambda$ be an admissible path, let $c$ be a corner of $\Lambda$ adjacent to consecutive edges $\theta_{1}, \theta_{2}$, and assume that $c$ is not a kink, ie $\theta_{2}-\theta_{1} \in(0, \pi]$. Define a new admissible path $\Lambda \backslash c$ of the same type as follows.

- If $\Lambda$ is an open admissible path defined on $I$, then $\Lambda \backslash c=\Lambda$ on $I \backslash\left[\theta_{1}, \theta_{2}\right]$. If $\Lambda$ is periodic or closed, then $\Lambda \backslash c=\Lambda$ on $\mathbb{R} \backslash p^{-1}\left[\theta_{1}, \theta_{2}\right]$.

- If $m$ and $m_{c}$ denote the multiplicity functions for $\Lambda$ and $\Lambda \backslash c$ respectively, then $m_{c}\left(\theta_{i}\right)=m\left(\theta_{i}\right)-1$ for $i=1,2$.

- If $\Lambda$ is an open admissible path, let $W \subset \mathbb{Z}^{2}$ be the set of lattice points enclosed by the triangle (or 2-gon when $\theta_{2}-\theta_{1}=\pi$ ) in the plane whose corners are

$$
\Lambda(c)-\left(\begin{array}{c}
x_{\theta_{1}} \\
y_{\theta_{1}}
\end{array}\right), \quad \Lambda(c), \quad \Lambda(c)+\left(\begin{array}{c}
x_{\theta_{2}} \\
y_{\theta_{2}}
\end{array}\right) .
$$

Then $\left.(\Lambda \backslash c)\right|_{\left(\theta_{1}, \theta_{2}\right)}$ traverses counterclockwise the boundary of the convex hull of $W \backslash\{\Lambda(c)\}$, except for the edge from

$$
\Lambda(c)+\left(\begin{array}{l}
x_{\theta_{2}} \\
y_{\theta_{2}}
\end{array}\right) \text { to } \Lambda(c)-\left(\begin{array}{l}
x_{\theta_{1}} \\
y_{\theta_{1}}
\end{array}\right) .
$$

If $\Lambda$ is periodic or closed, then $\Lambda \backslash c$ is defined the same way on each component of $p^{-1}\left(\theta_{1}, \theta_{2}\right)$.

We say that $\Lambda \backslash c$ is obtained from $\Lambda$ by rounding the corner at $c$.

Example 2.5 Suppose $\Lambda$ is a closed admissible path of rotation number 1, corresponding to the boundary of a convex polygonal region $P$. If $c$ is a corner of $\Lambda$ mapping to a corner $\Lambda(c)$ of $P$, then $\Lambda \backslash c$ corresponds to the boundary of the convex hull of the set of lattice points in $P \backslash\{\Lambda(c)\}$.

\subsection{A partial order on admissible paths}

We now introduce a partial order on the set of admissible paths and collect some useful facts about it. This partial order plays a fundamental role both in our computation of the homology of the chain complexes $C_{*}(2 \pi n ; \Gamma)$ and in the connection with pseudoholomorphic curves in $\mathbb{R} \times T^{3}$.

Let $\Lambda$ and $\Lambda^{\prime}$ be admissible paths of the same type, as defined in Section 2.1. 
Definition 2.6 We say that $\Lambda^{\prime}$ is to the left of $\Lambda$, and we write $\Lambda^{\prime} \leq \Lambda$, if

$$
\operatorname{det}\left(\begin{array}{l}
\cos t \\
\sin t
\end{array} \Lambda^{\prime}(t)-\Lambda(t)\right) \geq 0
$$

for all $t$ in $I$ (if $\Lambda, \Lambda^{\prime}$ are open) or $\mathbb{R}$ (if $\Lambda, \Lambda^{\prime}$ are periodic or closed).

Note that the left side of (11) is defined even if $t$ is an edge of $\Lambda$ or $\Lambda^{\prime}$, because by equation (9) or (10), the determinant in (11) extends to a continuous function defined for all $t$ in $I$ or $\mathbb{R}$.

Remark 2.7 Let $L_{t}$ and $L_{t}^{\prime}$ denote the oriented lines through $\Lambda(t)$ and $\Lambda^{\prime}(t)$ respectively in the direction $(\cos t, \sin t)$. Then $\Lambda^{\prime} \leq \Lambda$ iff for all $t$, the oriented line $L_{t}^{\prime}$ is (not necessarily strictly) to the left of $L_{t}$ in the usual sense.

Proposition $2.8 \leq$ is a partial order.

Proof By (11), we have $\Lambda \leq \Lambda$, and if $\Lambda_{1} \leq \Lambda_{2}$ and $\Lambda_{2} \leq \Lambda_{3}$, then $\Lambda_{1} \leq \Lambda_{3}$.

Now suppose that $\Lambda^{\prime} \leq \Lambda$ and $\Lambda \leq \Lambda^{\prime}$; we must show that $\Lambda^{\prime}=\Lambda$. We have

$$
\operatorname{det}\left(\begin{array}{c}
\cos t \\
\sin t
\end{array} \Lambda^{\prime}(t)-\Lambda(t)\right)=0
$$

for all $t$ for which $\Lambda, \Lambda^{\prime}$ are defined. Now $\Lambda^{\prime}(t)$ and $\Lambda(t)$ are locally constant and defined on the complement of the edges of $\Lambda$ and $\Lambda^{\prime}$. Since the vector $(\cos t, \sin t)$ rotates as $t$ varies, the vanishing of the determinant implies that $\Lambda^{\prime}(t)-\Lambda(t)=0$ on each interval between edges of $\Lambda$ and $\Lambda^{\prime}$.

Example 2.9 For closed admissible paths $\Lambda$ and $\Lambda^{\prime}$ of rotation number 1, corresponding to the boundaries of convex polygonal regions $P$ and $P^{\prime}$, we have

$$
\Lambda^{\prime} \leq \Lambda \Longleftrightarrow P^{\prime} \subset P \text {. }
$$

Proof $(\Leftarrow)$ Suppose $P^{\prime} \subset P$. We need to show that $\Lambda^{\prime} \leq \Lambda$; equivalently, for each $t$ the point $\Lambda^{\prime}(t)$ is to the left of the line $L_{t}$. But this holds because convexity of $P$ implies that all of $P$ is to the left of $L_{t}$, and $P^{\prime} \subset P$.

$(\Rightarrow)$ Suppose $P^{\prime} \not \subset P$. Let $x \in P^{\prime}$ maximize distance to $P$. Let $y \in P$ minimize distance to $x$. If $x$ is a corner of $P^{\prime}$ and $y$ is a corner of $P$, then there exists $t \in \mathbb{R} / 2 \pi \mathbb{Z}$ such that $x=\Lambda^{\prime}(t)$ and $y=\Lambda(t)$, and $x$ is strictly to the right of the line $L_{t}$, so

$$
\operatorname{det}\left(\begin{array}{l}
\cos t \\
\sin t
\end{array} \Lambda^{\prime}(t)-\Lambda(t)\right)<0
$$


If $x$ is in the interior of an edge of $P^{\prime}$ and $y$ is in the interior of an edge of $P$, then these edges both point in the same direction $(\cos t, \sin t)$ and the inequality (12) still holds. If $x$ is a corner of $P^{\prime}$ and $y$ is in the interior of an edge of $P$ with tangent direction $(\cos t, \sin t)$ then $x=\Lambda^{\prime}(t)$ and (12) still holds; likewise if just $y$ is a corner.

There is a useful characterization of the corner rounding operation in terms of the partial order $\leq$. Namely, $\Lambda \backslash c$ is the maximal admissible path which is to the left of $\Lambda$ and which does not "go through" the corner $c$ of $\Lambda$. More precisely:

Proposition 2.10 Let $\Lambda$ be an admissible path and let $c$ be a corner of $\Lambda$ which is not a kink. Then:

(a) $\Lambda \backslash c \leq \Lambda$.

(b) If $\Lambda^{\prime} \leq \Lambda$, and if $\Lambda^{\prime}$ disagrees with $\Lambda$ somewhere on the interval corresponding to $c$, then $\Lambda^{\prime} \leq \Lambda \backslash c$.

Lemma 2.11 Suppose $\Lambda^{\prime} \leq \Lambda$. Let $t_{1}, t_{2}$ be real numbers in the domain of $\Lambda, \Lambda^{\prime}$ with $t_{2}-t_{1} \in(0, \pi)$. Then the restriction of $\Lambda^{\prime}$ to $\left[t_{1}, t_{2}\right]$ maps to the wedge consisting of those $x \in \mathbb{R}^{2}$ that are to the left of $L_{t_{1}}$ and $L_{t_{2}}$.

Proof For $i=1,2$ and $t \in\left[t_{1}, t_{2}\right]$ define a piecewise constant function

$$
f_{i}(t):=\operatorname{det}\left(\begin{array}{c}
\cos t_{i} \\
\sin t_{i}
\end{array} \Lambda^{\prime}(t)-\Lambda\left(t_{i}\right)\right) .
$$

Note that this is well defined even if $t_{i}$ is an edge of $\Lambda$, as one can then take $\Lambda\left(t_{i}\right)$ to be any point on the corresponding line in $\mathbb{R}^{2}$. Now by equation (9) or (10) and our assumption that $t_{2}-t_{1} \in(0, \pi)$, we have

$$
\frac{d}{d t} f_{1}(t) \geq 0, \quad \frac{d}{d t} f_{2}(t) \leq 0
$$

for $t \in\left[t_{1}, t_{2}\right]$. On the other hand, since we assumed that $\Lambda^{\prime} \leq \Lambda$, putting $t=t_{i}$ into the definition of $\leq$ gives

$$
f_{1}\left(t_{1}\right) \geq 0, \quad f_{2}\left(t_{2}\right) \geq 0 .
$$

Therefore for all $t \in\left[t_{1}, t_{2}\right]$, we have $f_{1}(t), f_{2}(t) \geq 0$, which means that $\Lambda^{\prime}(t)$ is to the left of $L_{t_{1}}$ and $L_{t_{2}}$.

Proof of Proposition 2.10 To simplify notation we assume that $\Lambda$ is an open admissible path; the proof when $\Lambda$ is a closed or periodic admissible path works the same 
way. Let $\theta_{1}, \theta_{2}$ be the edges of $\Lambda$ adjacent to $c$. We assume that $\theta_{2}-\theta_{1}<\pi$ and leave the easier, extreme case when $\theta_{2}-\theta_{1}=\pi$ as an exercise.

(a) Equation (11) holds for $t \notin\left[\theta_{1}, \theta_{2}\right]$, as then $(\Lambda \backslash c)(t)=\Lambda(t)$ by definition of $\Lambda \backslash c$. Now suppose that $t \in\left[\theta_{1}, \theta_{2}\right]$. We need to show that $(\Lambda \backslash c)(t)$ is to the left of the line $L_{t}$. But this is clear since the entire triangle $W$ in the definition of $\Lambda \backslash c$ is to the left of the line $L_{t}$.

(b) Suppose $\Lambda^{\prime} \leq \Lambda$ and $\Lambda^{\prime} \not \leq \Lambda \backslash c$; we will show that $\Lambda^{\prime}$ agrees with $\Lambda$ on all of the interval $\left(\theta_{1}, \theta_{2}\right)$. For $i=1,2$ let $V$ be the wedge as in Lemma 2.11 for $t_{i}=\theta_{i}$. Let $P$ be the path in $V$ traced out by $\left.(\Lambda \backslash c)\right|_{\left[\theta_{1}, \theta_{2}\right]}$. The path $P$ separates $V$ into two components, one bounded and one unbounded. By Lemma 2.11, $\Lambda^{\prime}(t) \in V$ for all $t \in\left[\theta_{1}, \theta_{2}\right]$ for which $\Lambda^{\prime}(t)$ is defined. Since $\Lambda^{\prime} \not \leq \Lambda \backslash c$, there exists $t \in\left(\theta_{1}, \theta_{2}\right)$ such that if $L_{t}^{\prime \prime}$ denotes the oriented line through $(\Lambda \backslash c)(t)$ in the direction $(\cos t, \sin t)$, then $\Lambda^{\prime}(t)$ is strictly to the right of the line $L_{t}^{\prime \prime}$. Since $P$ is convex and to the left of $L_{t}^{\prime \prime}$, it follows that $\Lambda^{\prime}(t)$ is in the bounded component of $V \backslash P$. By definition of $\Lambda \backslash c$, there are no lattice points in the interior of the bounded component of $V \backslash P$, so we must have $\Lambda^{\prime}(t)=\Lambda(c)$. Then $\Lambda^{\prime}$ must agree with $\Lambda$ on the entire interval $\left(\theta_{1}, \theta_{2}\right)$, since if $\Lambda^{\prime}$ had any jumps on this interval, then by equation (9) for $\Lambda^{\prime}$ it would escape the wedge $V$.

The following proposition shows that the relation $\leq$ imposes strong restrictions in the presence of kinks.

Proposition 2.12 Suppose $\Lambda$ has a kink at $c$ and $\Lambda^{\prime} \leq \Lambda$. Then $\Lambda^{\prime}$ agrees with $\Lambda$ on the interval corresponding to $c$.

Proof Let $\theta_{1}, \theta_{2}$ be the edges of $\Lambda$ adjacent to $c$, and let $t \in\left(\theta_{1}, \theta_{2}\right)$; we must show that $\Lambda^{\prime}(t)=\Lambda(c)$. Since $\theta_{2}-\theta_{1}>\pi$, we can find $t_{0}$ with

$$
\theta_{1}<t_{0}<t<t_{0}+\pi<\theta_{2} \text {. }
$$

By Lemma 2.11, if

$$
t_{0}<t_{1}<t_{2}<t_{0}+\pi
$$

then $\Lambda^{\prime}\left[t_{1}, t_{2}\right]$ is contained in the wedge of $x \in \mathbb{R}^{2}$ to the left of $L_{t_{1}}$ and $L_{t_{2}}$. Taking the limit as $t_{1} \searrow t_{0}$ and $t_{2} \nearrow t_{0}+\pi$, we conclude that $\Lambda^{\prime}(t)$ is in the intersection of all such wedges, which is half of the line $L_{t_{0}}$. Now we can perturb $t_{0}$ so as to still satisfy equation (13), so $\Lambda^{\prime}(t)$ must lie on another nearby such line, and these two lines intersect only at the point $\Lambda(c)$, so $\Lambda^{\prime}(t)=\Lambda(c)$.

Finally, there is a sort of converse to Proposition 2.10 which characterizes the partial order $\leq$ in terms of the corner rounding operation. 
Proposition 2.13 Let $\Lambda, \Lambda^{\prime}$ be admissible paths of the same type. Then $\Lambda^{\prime} \leq \Lambda$ if and only if one can obtain $\Lambda^{\prime}$ from $\Lambda$ by a finite sequence of corner roundings.

The proof of this proposition uses induction on the "length" of an admissible path. If $\Lambda$ is an admissible path, define its length $\mathcal{A}(\Lambda)$ to be the sum of the lengths of its edges:

$$
\mathcal{A}(\Lambda):=\sum_{\theta \in T} m(\theta) \sqrt{x_{\theta}^{2}+y_{\theta}^{2}} .
$$

Since the set of possible lengths of admissible paths (namely finite sums of square roots of nonnegative integers) is a discrete set of nonnegative real numbers, it is valid to perform induction on length.

Lemma 2.14 Rounding corners decreases length, ie if $c$ is not a kink of $\Lambda$ then

$$
\mathcal{A}(\Lambda \backslash c)<\mathcal{A}(\Lambda)
$$

Proof Let $\theta_{1}, \theta_{2}$ be the two edges adjacent to $c$; since $c$ is not a kink, $\theta_{2}-\theta_{1} \in(0, \pi]$. The lemma is immediate if $\theta_{2}-\theta_{1}=\pi$, as then

$$
\mathcal{A}(\Lambda \backslash c)=\mathcal{A}(\Lambda)-2 \sqrt{x_{\theta_{1}}^{2}+y_{\theta_{1}}^{2}} .
$$

So assume that $\theta_{2}-\theta_{1} \in(0, \pi)$. Let $P$ be the path in the plane consisting of the line segments from

$$
\Lambda(c)-\left(\begin{array}{c}
x_{\theta_{1}} \\
y_{\theta_{1}}
\end{array}\right) \text { to } \Lambda(c) \text { to } \Lambda(c)+\left(\begin{array}{c}
x_{\theta_{2}} \\
y_{\theta_{2}}
\end{array}\right) .
$$

In passing from $\Lambda$ to $\Lambda \backslash c, P$ is replaced by a path $Q$, which we regard as a continuous embedded path in the plane. We need to show that $P$ is longer than $Q$. Define a map

$$
f: Q \backslash\{\text { corners }\} \longrightarrow P
$$

as follows. If $x \in Q$ is not a corner, let $L_{x}$ be the line through $x$ perpendicular to $Q$, and define $f(x)=L_{x} \cap P$. Since $Q$ is convex, the map $f$ is injective (although not surjective if $Q$ has corners) and increases length.

Proof of Proposition 2.13 $\quad(\Leftarrow)$ This follows immediately from Proposition 2.10(a) and the transitivity of $\leq$.

$\Leftrightarrow$ Suppose $\Lambda^{\prime} \leq \Lambda$; we need to show that there is a sequence of corner roundings from $\Lambda$ to $\Lambda^{\prime}$. If $\Lambda^{\prime}=\Lambda$ there is nothing to prove. If $\Lambda^{\prime} \neq \Lambda$, then there is a corner $c$ of $\Lambda$ such that $\Lambda^{\prime}$ disagrees with $\Lambda$ somewhere on the interval corresponding to $c$. By Propositions 2.12 and 2.10(b), $c$ is not a kink of $\Lambda$ and $\Lambda^{\prime} \leq \Lambda \backslash c$. By Lemma 2.14, $\mathcal{A}(\Lambda \backslash c)<\mathcal{A}(\Lambda)$, so by induction on length there is a sequence of corner roundings from $\Lambda \backslash c$ to $\Lambda^{\prime}$. 


\section{Polygon complexes}

In this section we define the combinatorial chain complexes of interest, and we prove that the combinatorial differential $\delta$ has degree -1 and satisfies $\delta^{2}=0$.

\subsection{The chain complex $C_{*}(2 \pi n ; \Gamma)$ and variants}

Fix a positive integer $n$ and an integer vector $\Gamma \in \mathbb{Z}^{2}$. We now define the combinatorial chain complex $C_{*}(2 \pi n ; \Gamma)$, and its variants $\widetilde{C}_{*}(2 \pi n ; \Gamma)$ and $\bar{C}_{*}(2 \pi n ; \Gamma)$ which are relevant to the embedded contact homology of $T^{3}$.

\subsubsection{The generators}

Definition 3.1 $C_{*}(2 \pi n ; \Gamma)$ is the $\mathbb{Z}$-module generated by triples $(\Lambda, l, o)$, where:

- $\Lambda$ is a periodic admissible path with rotation number $n$ and period $\Gamma$, as defined in Section 2.1. (We say that $(\Lambda, l, o)$ has "underlying admissible path $\Lambda$ ".)

- $l$ is a labeling of each of the edges of $\Lambda$ by ' $e$ ' or ' $h$ '.

- $o$ is an ordering of the set of edges that are labeled ' $h$ '.

We impose the relations that $(\Lambda, l, o)=\left(\Lambda, l, o^{\prime}\right)$ if the orderings $o$ and $o^{\prime}$ differ by an even permutation, and $(\Lambda, l, o)=-\left(\Lambda, l, o^{\prime}\right)$ if $o$ and $o^{\prime}$ differ by an odd permutation. Thus $C_{*}(2 \pi n ; \Gamma)$ is a free $\mathbb{Z}$-module with one generator (with no canonical sign) for each pair $(\Lambda, l)$.

3.1.2 The grading We now define the grading on $C_{*}(2 \pi n ; \Gamma)$. If $\alpha=(\Lambda, l, o)$ is a generator, consider the sum of the multiplicities of the edges,

$$
\ell(\alpha):=\sum_{\theta \in T} m(\theta)
$$

That is, $\ell(\alpha)$ is the number of lattice points traversed by the path $\Lambda=: \Lambda(\alpha)$, counted with repetitions, but modulo translation by $2 \pi n$. Let $\# h(\alpha)$ denote the number of edges of $\Lambda$ that are labeled ' $h$ '.

Definition 3.2 If $\Gamma=0$, define the index of a generator $\alpha$ to be

$$
I(\alpha):=2 \int_{\Lambda(\alpha)} x d y+\ell(\alpha)-\# h(\alpha) \in \mathbb{Z} .
$$

Here $\int_{\Lambda(\alpha)} x d y$ is the area, counted with multiplicity, enclosed by the polygonal path $\Lambda(\alpha)$; twice this area is an integer by Pick's formula. 
If $\Gamma=0$ and $n>1$ then the index can be any integer, since there are then admissible paths with negative area. (An example is shown in Section 2.1.) If $\Gamma \neq 0$ then there is no canonical absolute grading, only a relative grading which is defined as follows.

Definition 3.3 Let $\alpha$ and $\beta$ be generators of $C_{*}(2 \pi n ; \Gamma)$. Choose

$$
t \in \mathbb{R} \backslash p^{-1}(T(\alpha) \bigcup T(\beta))
$$

and let $\eta$ be any path in $\mathbb{R}^{2}$ from $\Lambda(\alpha)(t)$ to $\Lambda(\beta)(t)$. Let $\eta^{\prime}$ denote the the translation of $\eta$ by $\Gamma$. Define the composite loop

$$
P:=\left.\Lambda(\alpha)\right|_{[t, t+2 \pi n]}+\eta^{\prime}-\left.\Lambda(\beta)\right|_{[t, t+2 \pi n]}-\eta .
$$

Define the relative index

$$
I(\alpha, \beta):=2 \int_{P} x d y+(\ell(\alpha)-\# h(\alpha))-(\ell(\beta)-\# h(\beta)) \in \mathbb{Z} .
$$

Note that if $\Gamma=0$, then $I(\alpha, \beta)=I(\alpha)-I(\beta)$. This still holds when $\Gamma \neq 0$ if one regards the index of a single generator as taking values in an affine space over $\mathbb{Z}$. There is also a canonical mod 2 index

$$
I_{2}(\alpha):=\# h(\alpha) \bmod 2 .
$$

This satisfies $I_{2}(\alpha)-I_{2}(\beta) \equiv I(\alpha, \beta) \bmod 2$.

\subsubsection{The differential}

Definition 3.4 Define the differential

$$
\delta: C_{*}(2 \pi n ; \Gamma) \rightarrow C_{*-1}(2 \pi n ; \Gamma)
$$

as follows. Let $\alpha$ be a generator of $C_{*}(2 \pi n ; \Gamma)$. We define $\delta \alpha$ to be the signed sum of all ways to round a corner of $\alpha$ and "locally lose one $h$ ". More precisely, the differential coefficient $\langle\delta \alpha, \beta\rangle \neq 0$ if and only if:

- If $\alpha$ has underlying admissible path $\Lambda$, then $\beta$ has underlying admissible path $\Lambda \backslash c$, where $c$ is a corner of $\Lambda$ which is not a kink, ie between consecutive edges $\theta_{1}, \theta_{2}$ with $\theta_{2}-\theta_{1} \in(0, \pi]$.

- The edge labels of $\alpha$ and $\beta$ are identical for edges outside of the closed interval $\left[\theta_{1}, \theta_{2}\right]$, and either:

- Exactly one of the edges $\theta_{1}, \theta_{2}$ for $\alpha$ is labeled ' $h$ ', and all the edges in $\left[\theta_{1}, \theta_{2}\right]$ for $\beta$ are labeled ' $e$ ', or: 
- Both of the edges $\theta_{1}, \theta_{2}$ for $\alpha$ are labeled ' $h$ ', and exactly one of the edges in $\left[\theta_{1}, \theta_{2}\right]$ for $\beta$ is labeled ' $h$ '.

In this case $\langle\delta \alpha, \beta\rangle:= \pm 1$, with the sign determined as in Section 1.3.3.

3.1.4 Variants The group $\mathbb{Z}^{2}$ acts on the chain complex $C_{*}(2 \pi n ; \mathbb{Z})$ by translation in the plane. Thus there is a variant $\widetilde{C}_{*}(2 \pi n ; \Gamma)$, which is just $C_{*}(2 \pi n ; \Gamma)$ regarded as a $\mathbb{Z}\left[\mathbb{Z}^{2}\right]$-module. There is also a variant $\bar{C}_{*}(2 \pi n ; \Gamma)$ in which we mod out by translation of polygons. Formally,

$$
\bar{C}_{*}(2 \pi n ; 0)=\widetilde{C}_{*}(2 \pi n ; 0) \otimes_{\mathbb{Z}\left[\mathbb{Z}^{2}\right]} \mathbb{Z},
$$

where $\underline{\mathbb{Z}}$ is defined in Section 1.3.5. We regard $\bar{C}_{*}(2 \pi n ; \Gamma)$ as a $\mathbb{Z}$-module.

When

$$
\Gamma=\left(\begin{array}{l}
\Gamma_{1} \\
\Gamma_{2}
\end{array}\right) \neq 0,
$$

the relative grading on $\bar{C}_{*}(2 \pi n ; \Gamma)$ takes values in $\mathbb{Z} / 2 \operatorname{gcd}\left(\Gamma_{1}, \Gamma_{2}\right)$. The reason is that if $\alpha$ is a generator of $C_{*}(2 \pi n ; \Gamma)$ and if $\Psi_{w}$ denotes the translation in the plane by a vector

$$
w=\left(\begin{array}{l}
w_{1} \\
w_{2}
\end{array}\right) \in \mathbb{Z}^{2}
$$

then by the definition of the relative index (16), we have the "index ambiguity formula"

$$
I\left(\alpha, \Psi_{w} \alpha\right)=2 \operatorname{det}\left(\begin{array}{ll}
\Gamma_{1} & w_{1} \\
\Gamma_{2} & w_{2}
\end{array}\right)
$$

3.1.5 The auxiliary chain complex $C_{*}(\Lambda)$ We now introduce an auxiliary complex which will be used in the computation of the homology of $C_{*}(2 \pi n ; \Gamma)$. Let $\Lambda$ be an admissible path of any type (see the end of Section 2.1). Define a chain complex $C_{*}(\Lambda)$ as follows.

Definition 3.5 $C_{*}(\Lambda)$ is the $\mathbb{Z}$-module generated by triples $(\lambda, l, o)$ where $\lambda$ is an admissible path of the same type as $\Lambda$ with $\lambda \leq \Lambda, l$ is a labeling of the edges of $\lambda$ by ' $e$ ' or ' $h$ ', and $o$ is an ordering of the ' $h$ ' edges, with $(\lambda, l, o)= \pm(\lambda, l, o$ ') as in Definition 3.1. The differential $\delta$ on $C_{*}(\Lambda)$ is defined just as in Definition 3.4.

Note that $\delta$ sends $C_{*}(\Lambda)$ to itself, by Proposition 2.10(a) and transitivity of $\leq$. If $\Lambda \leq \Lambda^{\prime}$, then $C_{*}(\Lambda)$ is a subcomplex of $C_{*}\left(\Lambda^{\prime}\right)$. If $\Lambda$ is closed or periodic of rotation number $n$ and period $\Gamma$, then $C_{*}(\Lambda)$ is a subcomplex of $C_{*}(2 \pi n ; \Gamma)$. 
The chain complex $C_{*}(\Lambda)$ has a relative $\mathbb{Z}$-grading; if $\Lambda$ is open, this is defined by equation (16), with $P$ the difference between the two admissible paths. If $\Lambda$ is closed, or if $\Lambda$ is open and its initial and final endpoints agree, then $C_{*}(\Lambda)$ has a canonical absolute $\mathbb{Z}$-grading, defined by equation (15).

If $\Lambda$ is an open admissible path parametrized by an interval $I$, then the ordering of $I$ gives rise to a canonical ordering of the ' $h$ ' edges of any generator of $C_{*}(\Lambda)$, so we can regard $C_{*}(\Lambda)$ as generated by pairs $(\lambda, l)$.

3.1.6 The cycles $E_{\Lambda}$ and $H_{\Lambda} \quad$ For any admissible path $\Lambda$, the chain complex $C_{*}(\Lambda)$ contains two special cycles which will play a fundamental role in our calculations.

Definition 3.6 For any admissible path $\Lambda$, define $E_{\Lambda}, H_{\Lambda} \in C_{*}(\Lambda)$ as follows:

- $E_{\Lambda}$ is the path $\Lambda$ with all edges labeled ' $e$ '.

- $H_{\Lambda}$ is the sum of all ways of taking the path $\Lambda$ and labeling one edge ' $h$ ' and all other edges ' $e$ '. In particular, if $\Lambda$ has no edges, ie if $\Lambda$ is a constant path, then $H_{\Lambda}:=0$.

Note that since $E_{\Lambda}$ is a generator with no ' $h$ ' edge, and $H_{\Lambda}$ is a sum of generators each containing exactly one ' $h$ ' edge, there is no choice to make here in ordering the ' $h$ ' edges.

Lemma 3.7 If $\Lambda$ is any admissible path, then $\delta E_{\Lambda}=\delta H_{\Lambda}=0$.

Proof $E_{\Lambda}$ is automatically a cycle because it is a generator with no ' $h$ ' edges.

To see that $H_{\Lambda}$ is a cycle, let $\theta_{0}<\cdots<\theta_{k}$ be the edges of $\Lambda$, and for $0 \leq i \leq k$ let $\alpha_{i}$ be the summand in $H_{\Lambda}$ in which the edge $\theta_{i}$ is labeled ' $h$ '. Let $c_{i}$ be the corner between $\theta_{i-1}$ and $\theta_{i}$. If $0<i<k$ and if $c_{i}$ and $c_{i+1}$ are not kinks, then by the definition of $\delta$,

$$
\delta \alpha_{i}=E_{\Lambda \backslash c_{i}}-E_{\Lambda \backslash c_{i+1}} .
$$

A modified version of this formula holds for any $0 \leq i \leq k$, where the first term on the right is omitted if $c_{i}$ is a kink or $i=0$, and the second term on the right is omitted if $c_{i+1}$ is a kink or $i=k$. Thus $\delta H_{\Lambda}=\sum_{i=0}^{k} \delta \alpha_{i}$ consists of two copies of $E_{\Lambda \backslash c}$ for each corner $c$ of $\Lambda$ which is not a kink, and these two copies appear with opposite sign. 
3.1.7 Concatenation It will be important in our calculations to consider concatenations of the cycles $E_{\Lambda}$ and $H_{\Lambda}$. Suppose that $\Lambda_{1}$ and $\Lambda_{2}$ are open admissible paths parametrized by closed intervals $I_{1}$ and $I_{2}$ such that the right endpoint of $I_{1}$ (resp. $\Lambda_{1}$ ) agrees with the left endpoint of $I_{2}$ (resp. $\Lambda_{2}$ ). Then we can concatenate $\Lambda_{1}$ and $\Lambda_{2}$ to obtain an open admissible path which we denote by $\Lambda_{1} \Lambda_{2}$, which is parametrized by $I_{1} \cup I_{2}$, and which has a corner $c$ at the concatenation point. There is a natural inclusion

$$
C_{*}\left(\Lambda_{1}\right) \otimes C_{*}\left(\Lambda_{2}\right) \longrightarrow C_{*}\left(\Lambda_{1} \Lambda_{2}\right)
$$

which concatenates generators at $c$, and which we denote by juxtaposition of symbols. For example, $E_{\Lambda_{1}} E_{\Lambda_{2}}=E_{\Lambda_{1} \Lambda_{2}}$.

The map (19) is in general not a chain map. If $\alpha$ and $\beta$ are generators of $C_{*}\left(\Lambda_{1}\right)$ and $C_{*}\left(\Lambda_{2}\right)$ respectively, then it follows from the definition of $\delta$ that

$$
\delta(\alpha \beta)=(-1)^{\# h(\beta)}(\delta \alpha) \beta+\alpha(\delta \beta)+\delta_{c}(\alpha \beta) .
$$

Here $\# h(\beta)$ denotes the number of edges of $\beta$ labeled ' $h$ ', and $\delta_{c}$ denotes the contribution to $\delta$, if any, from rounding at the concatenation corner $c$.

Lemma 3.8 If the concatenation corner $c$ of $\Lambda_{1} \Lambda_{2}$ is not a kink, then in $C_{*}\left(\Lambda_{1} \Lambda_{2}\right)$,

$$
\begin{aligned}
& \delta\left(E_{\Lambda_{1}} H_{\Lambda_{2}}\right)=E_{\Lambda_{1} \Lambda_{2} \backslash c}, \\
& \delta\left(H_{\Lambda_{1}} E_{\Lambda_{2}}\right)=-E_{\Lambda_{1} \Lambda_{2} \backslash c}, \\
& \delta\left(H_{\Lambda_{1}} H_{\Lambda_{2}}\right)=H_{\Lambda_{1} \Lambda_{2} \backslash c} .
\end{aligned}
$$

Moreover, for each of these equations $\delta x=y$, for each generator $\gamma$ in $y$, there is a unique generator $\beta$ in $x$ with $\langle\delta \beta, \gamma\rangle \neq 0$.

Proof By Lemma 3.7 and equation (20), in the left hand side of each equation, all terms involving rounding at corners other than $c$ cancel out. It follows directly from the definition of $\delta$ that rounding at $c$ gives the right hand side of each equation, and that the last sentence of the lemma holds.

\section{$3.2 \delta$ has degree -1 and $\delta^{2}=0$}

Consider the chain complex $C_{*}(2 \pi n ; \Gamma)$ or $C_{*}(\Lambda)$ with its differential $\delta$. We first check that $\delta$ has degree -1 . Let $\alpha$ and $\beta$ be generators of the chain complex.

Lemma 3.9 Suppose the admissible path underlying $\beta$ is obtained from that of $\alpha$ by rounding a corner. Then

$$
I(\alpha, \beta)=2-\# h(\alpha)+\# h(\beta) .
$$


Proof Let $P$ be the polygon in the plane whose oriented boundary moves forward along the two edges (or parts of edges) of $\alpha$ that are rounded to obtain $\beta$, and then backwards along the edges of $\beta$ that are created in the rounding process. Let $m$ denote the sum of the multiplicities of these new edges in $\beta$. By the definition of the relative index in equation (16),

$$
I(\alpha, \beta)=2 \int_{P} x d y+2-\# h(\alpha)-m+\# h(\beta) .
$$

Note that $P$ is a simple closed polygon (except in the extremal case when the angle of the corner rounded in $\alpha$ is $\pi$, in which case $P$ is a $2-$ gon). By Pick's formula, since there are no lattice points in the interior of $P$, we have

$$
2 \int_{P} x d y=m
$$

Combining the above two equations proves the lemma.

Corollary 3.10 $\delta$ decreases degree by 1 : if $\langle\delta \alpha, \beta\rangle \neq 0$, then $I(\alpha, \beta)=1$.

We now turn to the proof that $\delta^{2}=0$. An important part of this is to check that the roundings of an admissible path at two different corners commute. Let $a$ and $b$ be distinct corners of an admissible path $\Lambda$ (of any type). Suppose that $a$ is not a kink of $\Lambda$ so that $\Lambda \backslash a$ is defined. Then $b$ induces a corner $b^{\prime}$ of $\Lambda \backslash a$. The interval corresponding to $b^{\prime}$ contains the interval corresponding to $b$. From now on we denote $b^{\prime}$ simply by $b$.

Lemma 3.11 Let $a$ and $b$ be distinct corners of an admissible path $\Lambda$. Suppose that $\Lambda \backslash a \backslash b$ is defined (ie $a$ is not a kink of $\Lambda$ and $b$ is not a kink of $\Lambda \backslash a$ ). Then $\Lambda \backslash b \backslash a$ is defined and

$$
\Lambda \backslash a \backslash b=\Lambda \backslash b \backslash a .
$$

Proof We can assume that $a$ and $b$ are consecutive and that an edge between them has multiplicity 1. (Otherwise the lemma is obvious as then the roundings at $a$ and $b$ involve disjoint portions of the domain of $\Lambda$ and thus do not affect each other.) Without loss of generality, $a$ precedes $b$.

By Proposition 2.10(a), $\Lambda \backslash a \backslash b \leq \Lambda$. Moreover, $\Lambda \backslash a \backslash b$ disagrees with $\Lambda \backslash a$ on all of the interval corresponding to $b$ in $\Lambda \backslash a$, which contains the interval of $\Lambda$ corresponding to $b$. So by Propositions 2.10(b) and 2.12, $b$ is not a kink of $\Lambda$ and

$$
\Lambda \backslash a \backslash b \leq \Lambda \backslash b .
$$


Now $\Lambda \backslash a \backslash b$ disagrees with $\Lambda \backslash b$ on an initial segment of the interval corresponding to $a$ in $\Lambda$, hence on some of the interval corresponding to $a$ in $\Lambda \backslash b$, so by Propositions 2.10(b) and 2.12, $a$ is not a kink of $\Lambda \backslash b$ and

$$
\Lambda \backslash a \backslash b \leq \Lambda \backslash b \backslash a .
$$

Since $b$ is not a kink of $\Lambda$, and $a$ is not a kink of $\Lambda \backslash b$, the mirror image of the above argument shows that $\Lambda \backslash b \backslash a \leq \Lambda \backslash a \backslash b$. Since $\leq$ is a partial order, it follows that $\Lambda \backslash a \backslash b=\Lambda \backslash b \backslash a$.

Lemma 3.12 Let $\alpha, \beta, \gamma$ be generators of $C_{*}(2 \pi n ; \Gamma)$ or $C_{*}(\Lambda)$ with $\langle\delta \alpha, \beta\rangle$ and $\langle\delta \beta, \gamma\rangle$ nonzero. Then:

(a) there is a unique (up to sign) generator $\beta^{\prime} \neq \pm \beta$ with $\left\langle\delta \alpha, \beta^{\prime}\right\rangle,\left\langle\delta \beta^{\prime}, \gamma\right\rangle \neq 0$.

(b) For $\beta^{\prime}$ as in (a),

$$
\langle\delta \alpha, \beta\rangle\langle\delta \beta, \gamma\rangle+\left\langle\delta \alpha, \beta^{\prime}\right\rangle\left\langle\delta \beta^{\prime}, \gamma\right\rangle=0 .
$$

Proof Let $a$ denote the corner of $\alpha$ that is rounded to obtain $\beta$, and let $b$ denote the corner of $\beta$ that is rounded to obtain $\gamma$. We consider three cases.

Case 1 The corner $b$ in $\beta$ comes from a corner $b$ of $\alpha$ which is not adjacent to $a$ and thus not affected by the rounding at $a$. Then $\beta^{\prime}$ is obtained by performing the rounding at $b$ first.

Case 2 The corner $b$ is created by the rounding at $a$. (We include here the extreme case where $a$ and $b$ correspond to the same interval of length $\pi$.) Since $\langle\delta \alpha, \beta\rangle$ and $\langle\delta \beta, \gamma\rangle$ are nonzero, both edges of $\alpha$ adjacent to $a$ must be labeled ' $h$ ', and one edge of $\beta$ adjacent to $b$ must be labeled ' $h$ '. Then $\beta$ ' is obtained from $\beta$ by switching the labels of the edges adjacent to $b$.

Case 3 The corner $b$ in $\beta$ comes from a corner (also denoted by $b$ ) of $\alpha$ which is adjacent to $a$. Without loss of generality, $b$ comes after $a$ in $\alpha$; the case where $b$ comes before $a$ is proved by the mirror image of the argument below.

Any $\beta^{\prime}$ must be obtained from $\alpha$ by rounding at $a$ or $b$, since otherwise $\beta^{\prime}$ would be strictly to the left of $\alpha$ somewhere outside the union of the intervals corresponding to $a$ and $b$, and hence so would $\gamma$, which is a contradiction. Therefore this is a local problem and we may assume without loss of generality that the path underlying $\alpha$ is an open path $\Lambda_{1} \Lambda_{2} \Lambda_{3}$ (so we are in $C_{*}(\Lambda)$ for some open path $\Lambda$ ) where each $\Lambda_{i}$ is a single edge, the corner $a$ is between $\Lambda_{1}$ and $\Lambda_{2}$, and the corner $b$ is between $\Lambda_{2}$ and $\Lambda_{3}$. Since $\langle\delta \alpha, \beta\rangle$ and $\langle\delta \beta, \gamma\rangle$ are nonzero, at least two of the three edges of $\alpha$ must be labeled ' $h$ '. There are four ways this can happen. 
The first possibility is that

$$
\alpha=H_{\Lambda_{1}} H_{\Lambda_{2}} E_{\Lambda_{3}}
$$

By Lemmas 3.8 and 3.11,

$$
\begin{aligned}
\delta(\alpha) & =H_{\Lambda_{1} \Lambda_{2} \backslash a} E_{\Lambda_{3}}-H_{\Lambda_{1}} E_{\Lambda_{2} \Lambda_{3} \backslash b}, \\
\delta\left(H_{\Lambda_{1} \Lambda_{2} \backslash a} E_{\Lambda_{3}}\right) & =-E_{\Lambda_{1} \Lambda_{2} \Lambda_{3} \backslash a \backslash b}, \\
\delta\left(H_{\Lambda_{1}} E_{\Lambda_{2} \Lambda_{3} \backslash b}\right) & =-E_{\Lambda_{1} \Lambda_{2} \Lambda_{3} \backslash b \backslash a}=-E_{\Lambda_{1} \Lambda_{2} \Lambda_{3} \backslash a \backslash b} .
\end{aligned}
$$

These equations imply that $\delta^{2} \alpha=0$. Here there is a unique $\beta^{\prime}$, namely $\pm H_{\Lambda_{1}} E_{\Lambda_{2} \Lambda_{3} \backslash b}$. Likewise $\delta^{2} \alpha=0$ in the other three cases $\alpha=H_{\Lambda_{1}} E_{\Lambda_{2}} H_{\Lambda_{3}}$ etc. In each case the last sentence of Lemma 3.8 implies that $\beta^{\prime}$ is unique.

Corollary $3.13 \quad \delta^{2}=0$.

Proof Lemma 3.12 shows that $\left\langle\delta^{2} \alpha, \gamma\right\rangle=0$ for any generators $\alpha, \gamma$.

\section{The homology operation $U$}

We now define a degree -2 chain map

$$
U: \widetilde{C}_{*}(2 \pi n ; \Gamma) \longrightarrow \widetilde{C}_{*-2}(2 \pi n ; \Gamma) .
$$

The map $U$ will not appear very often in the rest of the paper. However we will need it in Section 8.1 to help compute $\bar{H}_{*}$, and it also has a geometric counterpart in the embedded contact homology of $T^{3}$ discussed in Section 12.1.4.

Fix $\theta \in \mathbb{R} / 2 \pi n \mathbb{Z}$ with $\tan \theta$ irrational. The admissible path underlying any generator $\alpha \in C_{*}(2 \pi n ; \Gamma)$ has a distinguished corner $c_{\theta}$, which is the component of $(\mathbb{R} / 2 \pi n \mathbb{Z}) \backslash$ $T$ containing $\theta$. The definition of $U$ is similar to the definition of $\delta$, but here we preserve the number of ' $h$ ' edges and only round at the distinguished corner.

Definition 4.1 For a generator $\alpha \in C_{*}(2 \pi n ; \Gamma)$, define $U_{\theta} \alpha$ to be the sum of all generators $\beta$ such that:

- The admissible path underlying $\beta$ is obtained from that of $\alpha$ by rounding the distinguished corner $c_{\theta}$.

- Of the edges created or shortened by the rounding process, let $\left\{\theta_{i}\right\}$ denote those edges coming before $\theta$, and let $\left\{\theta_{j}^{\prime}\right\}$ denote those edges coming after $\theta$. If the edge of $\alpha$ before $c_{\theta}$ is labeled ' $h$ ', then exactly one of the edges $\theta_{i}$ of $\beta$ is labeled ' $h$ '; otherwise all the edges $\theta_{i}$ are labeled ' $e$ '. Likewise, if the edge of $\alpha$ after $c_{\theta}$ is labeled ' $h$ ', then exactly one of the edges $\theta_{j}^{\prime}$ of $\beta$ is labeled ' $h$ '; otherwise all the edges $\theta_{j}^{\prime}$ are labeled ' $e$ '. 
- For all edges of $\alpha$ not adjacent to $c_{\theta}$, the corresponding edges of $\beta$ have the same labels as the corresponding edges of $\alpha$. The ordering of the ' $h$ ' edges of $\beta$ is induced from the ordering of the ' $h$ ' edges of $\alpha$ under the obvious bijection between them.

It is implicit above that $U_{\theta} \alpha=0$ when $c_{\theta}$ is a kink in $\alpha$. When a fixed $\theta$ is understood, we write $U:=U_{\theta}$.

Proposition $4.2 \quad$ (a) If $\langle U \alpha, \beta\rangle \neq 0$, then $I(\alpha, \beta)=2$.

(b) $U x=x U$, and $U y=y U$, where $x$ and $y$ denote translation in the $x$-and $y$-directions.

(c) If the distinguished corner $c_{\theta}$ of $\Lambda$ is not a kink, then

$$
U\left(E_{\Lambda}\right)=E_{\Lambda \backslash c_{\theta}}, \quad U\left(H_{\Lambda}\right)=H_{\Lambda \backslash c_{\theta}} .
$$

Proof Property (a) follows from Lemma 3.9. Properties (b) and (c) are immediate from the definition of $U$.

Proposition 4.3 $U$ is a chain map: $\delta U=U \delta$.

Proof This will become clear after we compute $U$ of a concatenation of $E_{\Lambda}$ 's and $H_{\Lambda}$ 's. Let $\Lambda$ be a closed or periodic admissible path of rotation number $n$ and period $\Gamma$. Suppose that $\Lambda$ is a cyclic concatenation

$$
\Lambda=\Lambda_{1} \cdots \Lambda_{k}
$$

where $\Lambda_{i}$ is an open admissible path parametrized by the interval $\left[\theta_{i-1}, \theta_{i}\right] ; \Lambda_{i}\left(\theta_{i}\right)=$ $\Lambda_{i+1}\left(\theta_{i}\right)$, for $i=1, \ldots, k-1 ; \theta_{k}=\theta_{0}+2 \pi n$; and $\Lambda_{k}\left(\theta_{k}\right)=\Lambda_{0}\left(\theta_{0}\right)+\Gamma$. Let $c_{0}, c_{1}, \ldots, c_{k}=c_{0}$ denote the concatenation corners, regarded as open intervals in $\mathbb{R} / 2 \pi n \mathbb{Z}$. If $c_{\theta}$ is not a kink of $\Lambda$, then we can write $\Lambda \backslash c_{\theta}$ as a cyclic concatenation

$$
\Lambda \backslash c_{\theta}=\Lambda_{1}^{\prime} \cdots \Lambda_{k}^{\prime}
$$

where

$$
\Lambda_{i}^{\prime}:=\left\{\begin{array}{cl}
\left.\left(\Lambda_{i-1} \Lambda_{i} \backslash c_{\theta}\right)\right|_{\left[\theta, \theta_{i}\right]}, & \theta \in c_{i-1}, \\
\Lambda_{i} \backslash c_{\theta}, & \max \left(c_{i-1}\right)<\theta<\min \left(c_{i}\right), \\
\left.\left(\Lambda_{i} \Lambda_{i+1} \backslash c_{\theta}\right)\right|_{\left[\theta_{i-1}, \theta\right]}, & \theta \in c_{i}, \\
\Lambda_{i}, & \text { otherwise. }
\end{array}\right.
$$

(Here we interpret $\Lambda_{0}$ and $\Lambda_{k+1}$ as appropriate translates of $\Lambda_{k}$ and $\Lambda_{1}$ respectively.) The decomposition (22) is chosen so that if the distinguished corner $c_{\theta}$ agrees with a concatenation corner $c_{i}$ in (21), then the same is true for (22); and otherwise the 
concatenation corners of (21) and (22) agree. For each $i=1, \ldots, k$, let $X_{\Lambda_{i}}$ denote either $E_{\Lambda_{i}}$ or $H_{\Lambda_{i}}$, and let $X_{\Lambda_{i}^{\prime}}$ denote $E_{\Lambda_{i}^{\prime}}$ or $H_{\Lambda_{i}^{\prime}}$ respectively. Then it follows from the definition of $U$ that

$$
U\left(X_{\Lambda_{1}} \cdots X_{\Lambda_{k}}\right)=\left\{\begin{array}{cl}
X_{\Lambda_{1}^{\prime}} \cdots X_{\Lambda_{k}^{\prime}}, & c_{\theta} \text { not a kink of } \Lambda \\
0, & \text { otherwise. }
\end{array}\right.
$$

Also if $k=1$ then $\delta X_{\Lambda_{1}}=0$ by Lemma 3.7; and if $k>1$, then as in Lemma 3.8,

$$
\delta\left(X_{\Lambda_{1}} \cdots X_{\Lambda_{k}}\right)=\sum_{i=1}^{k} \pm X_{\Lambda_{1}} \cdots X_{\Lambda_{i-1}} Y_{\Lambda_{i} \Lambda_{i+1} \backslash c_{i}} X_{\Lambda_{i+2}} \cdots X_{\Lambda_{k}}
$$

Here

$$
Y_{\Lambda_{i} \Lambda_{i+1} \backslash c}:= \begin{cases}E_{\Lambda_{i} \Lambda_{i+1} \backslash c}, & \text { if } X_{\Lambda_{i}}=E_{\Lambda_{i}} \text { and } X_{\Lambda_{i+1}}=H_{\Lambda_{i+1}}, \\ E_{\Lambda_{i} \Lambda_{i+1} \backslash c}, & \text { if } X_{\Lambda_{i}}=H_{\Lambda_{i}} \text { and } X_{\Lambda_{i+1}}=E_{\Lambda_{i+1}}, \\ H_{\Lambda_{i} \Lambda_{i+1} \backslash c}, & \text { if } X_{\Lambda_{i}}=H_{\Lambda_{i}} \text { and } X_{\Lambda_{i+1}}=H_{\Lambda_{i+1}},\end{cases}
$$

if $c_{i}$ is not a kink of $\Lambda_{i} \Lambda_{i+1}$, and $Y_{\Lambda_{i} \Lambda_{i+1} \backslash c}:=0$ otherwise.

Now any generator of $C_{*}(2 \pi n ; \Gamma)$ can be written as a cyclic concatenation $X_{\Lambda_{1}} \cdots X_{\Lambda_{k}}$ where each $\Lambda_{i}$ has one edge. Hence to prove that $\delta U=U \delta$, by equations (24) and (25) it suffices to show that

$$
\left(\Lambda_{i}^{\prime} \Lambda_{i+1}^{\prime}\right) \backslash c_{i}=\left(\Lambda_{i} \Lambda_{i+1} \backslash c_{i}\right)^{\prime}
$$

and that one side of this equation is defined if and only if the other side is. This follows directly from equation (23) and Lemma 3.11 applied to $c_{i}$ and $c_{\theta}$.

We now consider the dependence of $U_{\theta}$ on $\theta$.

Definition 4.4 Let $\theta_{1}, \theta_{2} \in \mathbb{R} / 2 \pi n \mathbb{Z}$ with $\tan \theta_{1}, \tan \theta_{2}$ irrational. Define a $\mathbb{Z}\left[\mathbb{Z}^{2}\right]-$ linear map

$$
K_{\theta_{1}, \theta_{2}}: \widetilde{C}_{*}(2 \pi n ; \Gamma) \longrightarrow \widetilde{C}_{*-1}(2 \pi n ; \Gamma)
$$

as follows. If $\alpha$ is a generator of $\widetilde{C}_{*}(2 \pi n ; \Gamma)$, then $K_{\theta_{1}, \theta_{2}}(\alpha)$ is the sum of all ways of relabeling an ' $e$ ' edge of $\alpha$ in between $\theta_{1}$ and $\theta_{2}$ by ' $h$ ' and making this edge last in the ordering of the ' $h$ ' edges.

Proposition $4.5 \delta K_{\theta_{1}, \theta_{2}}+K_{\theta_{1}, \theta_{2}} \delta=U_{\theta_{1}}-U_{\theta_{2}}$. 
Corollary 4.6 The induced homomorphisms on homology

$$
\begin{aligned}
\left(U_{\theta}\right)_{*}: & \widetilde{H}_{*}(2 \pi n, \Gamma) \longrightarrow \widetilde{H}_{*-2}(2 \pi n ; \Gamma), \\
& \bar{H}_{*}(2 \pi n, \Gamma) \longrightarrow \bar{H}_{*-2}(2 \pi n ; \Gamma)
\end{aligned}
$$

do not depend on the choice of $\theta \in \mathbb{R} / 2 \pi n \mathbb{Z}$.

Proof of Proposition 4.5 First note that the statement of the proposition also makes sense in $C_{*}(\Lambda)$ where $\Lambda$ is an open admissible path and $\theta_{1}, \theta_{2} \in \mathbb{R}$. Here for $\theta \in \mathbb{R}$ and $\alpha \in C_{*}(\Lambda)$, it is understood that $U_{\theta} \alpha=0$ if $\theta$ is not contained in any corner of $\alpha$. Also, if $\theta_{1}>\theta_{2}$, then $K_{\theta_{1}, \theta_{2}}$ is understood to sum over ways of relabeling an ' $e$ ' edge that is greater than $\theta_{1}$ or less than $\theta_{2}$.

Now let $U_{i}$ and $K$ denote $U_{\theta_{i}}$ and $K_{\theta_{1}, \theta_{2}}$ respectively. We want to show that

$$
\delta K \alpha+K \delta \alpha=U_{1} \alpha-U_{2} \alpha
$$

for every generator $\alpha$ of $\widetilde{C}_{*}(2 \pi n ; \Gamma)$. Every term in this equation, up to edge labels, is obtained from $\alpha$ by rounding a single corner $c$ (depending on the term). Hence we need only check that for each corner $c$ of $\alpha$, the contributions to both sides of the equation involving rounding at $c$ agree. By the definition of $U$, contributions to the right hand side involving $c$ fix all edges not adjacent to $c$. The same is true for the left hand side, except for contributions in which $K$ relabels an edge that is neither adjacent to $c$ nor created by rounding at $c$; and by our sign conventions these terms cancel in pairs. Therefore it is enough to prove equation (26) in $C_{*}(\Lambda)$, where $\Lambda$ is an open admissible path with two edges and one corner $c$, and $\alpha$ is one of the four possible labelings of $\Lambda$. Also we can assume that $c$ is not a kink of $\Lambda$, since otherwise both sides of (26) immediately vanish.

Without loss of generality, $\theta_{1} \leq \theta_{2}$. The reason is that if $\theta_{1}>\theta_{2}$, then equation (26) follows by subtracting the case where $\theta_{1}$ and $\theta_{2}$ are switched from the case where $\theta_{1}<\theta_{2}$ and the interval $\left(\theta_{1}, \theta_{2}\right)$ contains the domain of the open path $\Lambda$.

Denote the four possibilities for $\alpha$ in the obvious manner by ee, $e h$, he, and $h h$. Let $\lambda:=\Lambda \backslash c$, let $\lambda^{<\theta_{i}}$ denote the part of $\lambda$ up to angle $\theta_{i}$, let $\lambda^{>\theta_{i}}$ denote the part of $\lambda$ after angle $\theta_{i}$, and let $\lambda^{\left(\theta_{1}, \theta_{2}\right)}$ denote the part of $\lambda$ between $\theta_{1}$ and $\theta_{2}$. For example, in this notation $\lambda=\lambda^{<\theta_{1}} \lambda^{\left(\theta_{1}, \theta_{2}\right)} \lambda^{>\theta_{1}}$. Let $E:=E_{\lambda}, H:=H_{\lambda}, H^{<\theta_{i}}:=H_{\lambda<\theta_{i}}$ etc. 
Suppose first that $\theta_{1}, \theta_{2} \in c$. Then $K \alpha=0$, and (26) follows from the computations

$$
\begin{aligned}
& U_{i}(e e)=E, \quad U_{i}(e h)=E^{<\theta_{i}} H^{>\theta_{i}}, \\
& U_{i}(h e)=H^{<\theta_{i}} E^{>\theta_{i}}, \quad U_{i}(h h)=H^{<\theta_{i}} H^{>\theta_{i}}, \\
& K \delta(e e)=K(0)=0, \\
& K \delta(e h)=K(E)=E^{<\theta_{1}} H^{\left(\theta_{1}, \theta_{2}\right)} E^{>\theta_{2}}, \\
& K \delta(h e)=K(-E)=-E^{<\theta_{1}} H^{\left(\theta_{1}, \theta_{2}\right)} E^{>\theta_{2}}, \\
& K \delta(h h)=K(H)=H^{<\theta_{1}} H^{\left(\theta_{1}, \theta_{2}\right)} E^{>\theta_{2}}-E^{<\theta_{1}} H^{\left(\theta_{1}, \theta_{2}\right)} H^{>\theta_{2}} .
\end{aligned}
$$

Note that in the last line, there are no terms with two ' $h$ ' edges in $\lambda$ between $\theta_{1}$ and $\theta_{2}$, because each such term arises twice in $K(H)$ with opposite sign.

The remaining cases where $\theta_{1} \notin c$ and/or $\theta_{2} \notin c$ follow by similar, straightforward calculations.

\section{Some preliminary homology calculations}

The closed admissible paths $\Lambda$ of rotation number $n$ form a directed set under the partial order $\leq$ defined in Section 2.3. Part of our strategy for computing $H_{*}(2 \pi n ; 0)$ is to realize $C_{*}(2 \pi n ; 0)$ as the direct limit of the subcomplexes $C_{*}(\Lambda)$ defined in Section 3.1.5, spanned by generators to the left of a given $\Lambda$. In this section, as a preliminary step, we calculate most of the homology $H_{*}(\Lambda)$ when $\Lambda$ is a closed admissible path of rotation number 1 . This homology is computed inductively using a long exact sequence introduced below. To carry out the induction, we will also need to calculate the homology $H_{*}(\Lambda)$ for certain open admissible paths $\Lambda$.

Throughout the homological calculations, the following decomposition will be useful.

Definition 5.1 Let $C_{*}^{(j)}(2 \pi n ; 0)$ denote the subcomplex of $C_{*}(2 \pi n ; 0)$ spanned by generators in which the index minus the number of ' $h$ ' edges equals $j$. Define $C_{*}^{(j)}(\Lambda)$ the same way if $\Lambda$ is a closed admissible path or an open admissible path whose endpoints agree, so that $C_{*}(\Lambda)$ has a canonical $\mathbb{Z}$-grading. Define $\widetilde{C}_{*}^{(j)}(2 \pi n ; 0)$ to be the corresponding subcomplex of $\widetilde{C}_{*}(2 \pi n ; 0)$.

When it should not cause confusion, we will use the same symbols to denote both cycles and the homology classes that they represent. 


\subsection{The rounding/breaking long exact sequence}

If $\Lambda$ is an admissible path, the homology $H_{*}(\Lambda)$ fits into a long exact sequence which provides a scheme for computing it by induction on the length of $\Lambda$. In the statement of the exact sequence we adopt the following:

Convention 5.2 If $c$ is a kink of $\Lambda$, so $\Lambda \backslash c$ is undefined, we interpret $H_{*}(\Lambda \backslash c):=0$.

There are two versions of this exact sequence depending on whether $\Lambda$ is open or closed/periodic. We first consider the case where $\Lambda$ is open.

Proposition 5.3 Let $\Lambda$ be an open admissible path. Suppose $\Lambda$ has a corner $c$ which splits it into open paths $\Lambda_{1}$ and $\Lambda_{2}$. Then there is a long exact sequence

$(27) \cdots \rightarrow H_{*}(\Lambda \backslash c) \rightarrow H_{*}(\Lambda) \rightarrow H_{*}\left(C_{*}\left(\Lambda_{1}\right) \otimes C_{*}\left(\Lambda_{2}\right)\right) \stackrel{\partial_{c}}{\longrightarrow} H_{*-1}(\Lambda \backslash c) \rightarrow \cdots$.

The first arrow is induced by inclusion, the second arrow is induced by projection (see below), and the map $\partial_{c}$ is defined by concatenating paths and computing the part of $\delta$ involving rounding at the corner $c$.

Proof Suppose first that $c$ is not a kink. Split $C_{*}(\Lambda)$ as the direct sum of two submodules where the generators are those whose underlying paths lie to the left of $\Lambda \backslash c$ and those whose paths do not. The former submodule is the subcomplex $C_{*}(\Lambda \backslash c)$. The latter submodule, which is not a subcomplex, is naturally isomorphic to $C_{*}\left(\Lambda_{1}\right) \otimes C_{*}\left(\Lambda_{2}\right)$, via concatenation of paths. For Proposition 2.10(b) shows that any generator of $C_{*}(\Lambda)$ not in $C_{*}(\Lambda \backslash c)$ is obtained by concatenating generators of $C_{*}\left(\Lambda_{1}\right)$ and $C_{*}\left(\Lambda_{2}\right)$, and the concatenation operation is clearly injective.

Define a differential on $C_{*}\left(\Lambda_{1}\right) \otimes C_{*}\left(\Lambda_{2}\right)$ by starting with the differential $\delta$ on $C_{*}(\Lambda)$ and discarding terms involving rounding at the corner $c$. We now have a short exact sequence of chain complexes

$$
0 \longrightarrow C_{*}(\Lambda \backslash c) \longrightarrow C_{*}(\Lambda) \longrightarrow C_{*}\left(\Lambda_{1}\right) \otimes C_{*}\left(\Lambda_{2}\right) \longrightarrow 0 .
$$

The above differential on $C_{*}\left(\Lambda_{1}\right) \otimes C_{*}\left(\Lambda_{2}\right)$ is given more explicitly as follows. If $\alpha$ and $\beta$ are generators of $C_{*}\left(\Lambda_{1}\right)$ and $C_{*}\left(\Lambda_{2}\right)$ respectively, and if $\# h(\beta)$ denotes the number of edges of $\beta$ labeled ' $h$ ', then by equation (20),

$$
\delta(\alpha \otimes \beta)=(-1)^{\# h(\beta)}(\delta \alpha) \otimes \beta+\alpha \otimes \delta \beta .
$$

This is the tensor product differential on $C_{*}\left(\Lambda_{2}\right) \otimes C_{*}\left(\Lambda_{1}\right)$, as determined by the canonical $\mathbb{Z} / 2$-grading on $C_{*}\left(\Lambda_{i}\right)$ given by $\# h \bmod 2$. Thus we obtain a long exact 
sequence on homology as claimed. The description of the connecting homomorphism is immediate from the definitions.

Suppose now that $c$ is a kink. Then by Proposition 2.12, every generator of $C_{*}(\Lambda)$ is obtained by concatenating generators of $C_{*}\left(\Lambda_{1}\right)$ and $C_{*}\left(\Lambda_{2}\right)$. As above, equation (20) then gives

$$
H_{*}(\Lambda) \simeq H_{*}\left(C_{*}\left(\Lambda_{1}\right) \otimes C_{*}\left(\Lambda_{2}\right)\right)
$$

Next suppose that $\Lambda$ is a closed or periodic admissible path with rotation number $n$. Let $c$ be a corner of $\Lambda$. We can cut $\Lambda$ at $c$ to obtain an open admissible path $\Lambda^{c}$ parametrized by the interval $\left(\theta_{0}, \theta_{0}+2 \pi n\right)$. Here $\theta_{0} \in \mathbb{R}$ is a lift of a point in $\mathbb{R} / 2 \pi n \mathbb{Z}$ in the interval corresponding to $c$. Note that the two endpoints of $\Lambda^{c}$ will differ by the period $\Gamma$ of $\Lambda$. If $\Gamma \neq 0$, then $\Lambda^{c}$ depends on the choice of $\theta_{0}$, and the different possibilities for $\Lambda^{c}$ differ by translation by multiples of $2 \pi n$ in the domain and $\Gamma$ in the range.

Proposition 5.4 If $\Lambda$ is a closed or periodic admissible path, if $c$ is a corner of $\Lambda$, and if $\Lambda^{c}$ is an open path obtained by cutting at $c$ as above, then there is a long exact sequence

$$
\cdots \longrightarrow H_{*}(\Lambda \backslash c) \longrightarrow H_{*}(\Lambda) \longrightarrow H_{*}\left(\Lambda^{c}\right) \stackrel{\partial_{c}}{\longrightarrow} H_{*-1}(\Lambda \backslash c) \longrightarrow \cdots .
$$

Proof This is a straightforward variant of Proposition 5.3.

\subsection{Convex open paths with distinct endpoints}

Definition 5.5 We say that an open admissible path $\Lambda$ is convex if it is parametrized by an interval of length $\leq 2 \pi$ and if it traverses a subset of the boundary of a convex polygon, possibly a $2-$ gon.

Proposition 5.6 Let $\Lambda$ be a convex open admissible path with distinct endpoints. Then $H_{*}(\Lambda)$ is the free $\mathbb{Z}$-module generated by the homology classes of $E_{\Lambda}$ and $H_{\Lambda}$ (see Section 3.1.5).

Proof When $\Lambda$ is a straight line, the proposition is trivial since $E_{\Lambda}$ and $H_{\Lambda}$ are the only two generators in $C_{*}(\Lambda)$, and the differential vanishes.

Now suppose that $\Lambda$ has a corner $c$. The corner $c$ splits $\Lambda$ into two open paths $\Lambda_{1}$ and $\Lambda_{2}$. Observe that $\Lambda_{1}, \Lambda_{2}$, and $\Lambda \backslash c$ all satisfy the hypotheses of the proposition. By induction on the length $\mathcal{A}(\Lambda)$ and using Lemma 2.14, we may assume that the proposition holds for $\Lambda_{1}, \Lambda_{2}$, and $\Lambda \backslash c$. 
By Proposition 5.3 there is a long exact sequence

$$
\cdots \rightarrow H_{*}(\Lambda \backslash c) \rightarrow H_{*}(\Lambda) \rightarrow H_{*}\left(\Lambda_{1}\right) \otimes H_{*}\left(\Lambda_{2}\right) \stackrel{\partial_{c}}{\longrightarrow} H_{*-1}(\Lambda \backslash c) \rightarrow \cdots .
$$

Here we have replaced $H_{*}\left(C_{*}\left(\Lambda_{1}\right) \otimes C_{*}\left(\Lambda_{2}\right)\right)$ by $H_{*}\left(\Lambda_{1}\right) \otimes H_{*}\left(\Lambda_{2}\right)$, since we know by inductive hypothesis that $H_{*}\left(\Lambda_{1}\right)$ and $H_{*}\left(\Lambda_{2}\right)$ have no torsion. We can replace this long exact sequence by the short exact sequence

$$
0 \longrightarrow \operatorname{Coker}\left(\partial_{c}\right) \longrightarrow H_{*}(\Lambda) \longrightarrow \operatorname{Ker}\left(\partial_{c}\right) \longrightarrow 0 .
$$

By Lemma 3.8, the connecting homomorphism $\partial_{c}$ is given by

$$
\begin{aligned}
& \partial_{c}\left(E_{\Lambda_{1}} \otimes E_{\Lambda_{2}}\right)=0, \\
& \partial_{c}\left(E_{\Lambda_{1}} \otimes H_{\Lambda_{2}}\right)=-\partial_{c}\left(H_{\Lambda_{1}} \otimes E_{\Lambda_{2}}\right)=E_{\Lambda \backslash c}, \\
& \partial_{c}\left(H_{\Lambda_{1}} \otimes H_{\Lambda_{2}}\right)=H_{\Lambda \backslash c} .
\end{aligned}
$$

Thus $\operatorname{Coker}\left(\partial_{c}\right)=0$, and $H_{*}(\Lambda) \simeq \operatorname{Ker}\left(\partial_{c}\right)$ is freely generated by the homology classes of the cycles $E_{\Lambda_{1}} E_{\Lambda_{2}}=E_{\Lambda}$ and $E_{\Lambda_{1}} H_{\Lambda_{2}}+H_{\Lambda_{1}} E_{\Lambda_{2}}=H_{\Lambda}$.

\subsection{Convex open paths with identical endpoints}

Our next task is to compute $H_{*}(\Lambda)$ where $\Lambda$ is a (non-constant) convex open admissible path whose two endpoints are the same lattice point $a$, so that $\Lambda$ traverses all of the boundary of a convex polygonal region $P_{\Lambda}$. Let $k_{\Lambda}$ denote the number of lattice points in $P_{\Lambda}$.

In this case a new homology generator appears. To describe it, let $b \neq a$ be a lattice point in $P_{\Lambda}$, and suppose that $b-a \in \mathbb{Z}^{2}$ is indivisible. Define

$$
Z_{1}(a, b) \in C_{*}(\Lambda)
$$

to be the 2-gon from $a$ to $b$ and back with both edges labeled ' $h$ ', and with the edge from $a$ to $b$ first in the ordering. By the definition of $\delta$, we have $\delta Z_{1}(a, b)=0$. It is not hard to show that for the open path $\Lambda$, the homology class of $Z_{1}(a, b)$ in $C_{*}(\Lambda)$ does not depend on $b$, although we will not need this.

Proposition 5.7 Let $\Lambda$ be a nonconstant convex open admissible path whose two endpoints are the same lattice point $a$. Then $H_{*}(\Lambda)$ is freely generated by the homology classes of $E_{\Lambda}, H_{\Lambda}$, and $Z_{1}(a, b)$ for a single $b$ with $b-a$ indivisible.

Proof We use induction on $k_{\Lambda}$, the number of lattice points in $P_{\Lambda}$.

If $k_{\Lambda}=2$, then the chain complex $C_{*}(\Lambda)$ has five generators: four 2-gons which we denote in the obvious manner by $e e, e h, h e$, and $h h$; and the constant path at $a$ which 
we denote by $p(a)$. The differential is given by $\delta(e e)=0, \delta(e h)=-\delta(h e)=p(a)$, $\delta(h h)=0$, and $\delta(p(a))=0$. The proposition in this case follows by inspection.

If $k_{\Lambda}>2$, let $c$ be a corner of $\Lambda$, which cuts $\Lambda$ into convex open paths $\Lambda_{1}$ and $\Lambda_{2}$ with distinct endpoints. We may inductively assume that the proposition holds for $\Lambda \backslash c$. By Proposition 5.6, $H_{*}\left(\Lambda_{i}\right)$ is freely generated by the homology classes of $E_{\Lambda_{i}}$ and $H_{\Lambda_{i}}$. By Proposition 5.3, there is a long exact sequence

$$
\cdots \rightarrow H_{*}(\Lambda \backslash c) \rightarrow H_{*}(\Lambda) \rightarrow H_{*}\left(\Lambda_{1}\right) \otimes H_{*}\left(\Lambda_{2}\right) \stackrel{\partial_{c}}{\longrightarrow} H_{*-1}(\Lambda \backslash c) \rightarrow \cdots .
$$

Here we have used the fact that $H_{*}\left(\Lambda_{i}\right)$ is free to commute homology and tensor product in the third term. The above long exact sequence gives rise to a short exact sequence

$$
0 \longrightarrow \operatorname{Coker}\left(\partial_{c}\right) \longrightarrow H_{*}(\Lambda) \longrightarrow \operatorname{Ker}\left(\partial_{c}\right) \longrightarrow 0 .
$$

The connecting homomorphism $\partial_{c}$ is again computed by the equations (30). Thus $\operatorname{Ker}\left(\partial_{c}\right)$ is freely generated by the homology classes of $E_{\Lambda}$ and $H_{\Lambda}$ as before, while now Coker $\left(\partial_{c}\right)$ is freely generated by the homology class of $Z_{1}(a, b)$.

\subsection{Closed paths of rotation number 1}

We now compute most of $H_{*}(\Lambda)$ where $\Lambda$ is a closed admissible path with rotation number 1. Again, $\Lambda$ traverses the boundary of a convex polygonal region $P_{\Lambda}$; let $k_{\Lambda}$ denote the number of lattice points in $P_{\Lambda}$.

If $\lambda \leq \Lambda$ is another closed admissible path with rotation number 1 , then $E_{\lambda}$ and $H_{\lambda}$ are cycles in $C_{*}(\Lambda)$. (Recall that when $\lambda$ is a constant path, $H_{\lambda}$ is defined to be zero.) We have the following relations between these cycles.

Lemma 5.8 Let $\Lambda$ be a closed admissible path with rotation number 1 and let $\lambda_{1}, \lambda_{2} \leq$ $\Lambda$ with $k_{\lambda_{1}}=k_{\lambda_{2}}=k \geq 1$. Then in the homology $H_{*}(\Lambda)$ we have

$$
\begin{aligned}
& E_{\lambda_{1}}=E_{\lambda_{2}}, \\
& H_{\lambda_{1}}=H_{\lambda_{2}} .
\end{aligned}
$$

Proof If $\lambda_{1}=\Lambda$ then necessarily $\lambda_{2}=\Lambda$ and the lemma is trivial. If $\lambda_{1} \neq \Lambda$ then by Proposition 2.13 there exist corners $a_{1}, a_{2}$ of $\Lambda$ with $\lambda_{i} \leq \Lambda \backslash a_{i}$. By induction on $k_{\Lambda}$, we may assume that the lemma holds for $\Lambda \backslash a_{i}$. Consequently, it is enough to choose convenient $\lambda_{i} \leq \Lambda \backslash a_{i}$ with $k_{\lambda_{i}}=k$ and verify the relations (31) and (32). Without loss of generality, $a_{1}$ and $a_{2}$ are consecutive corners of $\Lambda$ with $a_{1}$ coming first. 
Choose any $\mu \leq \Lambda$ with $k_{\mu}=k+1$. The path $\mu$ has at most one edge $\theta$ whose initial corner maps to the same lattice point as $a_{1}$ and/or whose final corner maps to the same lattice point as $a_{2}$. If $\mu$ has no such edge, let $\theta$ be an arbitrary edge of $\mu$. Let $b_{1}$ and $b_{2}$ denote the corners of $\mu$ before and after the edge $\theta$. Since $\mu$ is a closed admissible path of rotation number 1 , it contains no kinks. Choose $\lambda_{i}:=\mu \backslash b_{i}$; then $\lambda_{i} \leq \Lambda \backslash a_{i}$.

Let $\alpha$ be the generator of $C_{*}(\Lambda)$ with underlying path $\mu$ with the edge $\theta$ labeled ' $h$ ' and all other edges labeled ' $e$ '. Let $\beta \in C_{*}(\Lambda)$ be the chain obtained from $\alpha$ by summing over all ways of relabeling one of the ' $e$ ' edges by ' $h$ ' and ordering it first. Then similarly to Lemma 3.8,

$$
\begin{aligned}
& \delta \alpha=E_{\lambda_{1}}-E_{\lambda_{2}}, \\
& \delta \beta=H_{\lambda_{1}}-H_{\lambda_{2}} .
\end{aligned}
$$

Hence (31) and (32) hold in homology.

Note that by the index formula (6), the indices of the above generators are

$$
\begin{aligned}
& I\left(E_{\lambda}\right)=2\left(k_{\lambda}-1\right), \\
& I\left(H_{\lambda}\right)=2\left(k_{\lambda}-1\right)-1, \quad k_{\lambda}>1 .
\end{aligned}
$$

Proposition 5.9 Let $\Lambda$ be a closed admissible path of rotation number 1. Then:

(a) $H_{i}^{(-2)}(\Lambda)=0$ for $i \neq 0$. (See Definition 5.1.)

(b)

$$
\bigoplus_{j \neq-2} H_{i}^{(j)}(\Lambda) \simeq \begin{cases}\mathbb{Z}, & 0 \leq i \leq 2\left(k_{\Lambda}-1\right), \\ 0, & \text { otherwise. }\end{cases}
$$

This is generated by the homology classes of $E_{\lambda}$ for $\lambda \leq \Lambda$ and $H_{\lambda}$ for $\lambda \leq \Lambda$ nonconstant, with the relations (31) and (32).

Proof We use induction on $k_{\Lambda}$. If $k_{\Lambda}=1$ then $C_{*}(\Lambda)$ has only the single generator $E_{\Lambda}$ and the result is immediate. Suppose $k_{\Lambda}>1$, let $c$ be a corner of $\Lambda$, and assume that the proposition holds for $\Lambda \backslash c$. By Proposition 5.4 there is a long exact sequence in homology

$$
\cdots \longrightarrow H_{*}(\Lambda \backslash c) \longrightarrow H_{*}(\Lambda) \longrightarrow H_{*}\left(\Lambda^{c}\right) \stackrel{\partial_{c}}{\longrightarrow} H_{*-1}(\Lambda \backslash c) \longrightarrow \cdots
$$

By Proposition 5.7, $H_{*}\left(\Lambda^{c}\right)$ is freely generated by $E_{\Lambda^{c}}, H_{\Lambda^{c}}$, and $Z_{1}(c, b)$ for a single $b$ with $b-c$ indivisible. By regarding the open paths as closed paths, these cycles lift to cycles in $C_{*}(\Lambda)$, namely $E_{\Lambda}, H_{\Lambda}$, and a third cycle which we will also 
call $Z_{1}(c, b)$. Hence the connecting homomorphism $\partial_{c}$ vanishes, and we have a short exact sequence

$$
0 \longrightarrow H_{*}^{(j)}(\Lambda \backslash c) \longrightarrow H_{*}^{(j)}(\Lambda) \longrightarrow H_{*}^{(j)}\left(\Lambda^{c}\right) \longrightarrow 0 .
$$

The index calculation (33) implies that $E_{\Lambda}$ and $H_{\Lambda}$ have $j \geq 0$, while $Z_{1}(c, b)$ has $j=-2$. Also, the definition of the index implies that $I\left(Z_{1}(c, b)\right)=0$. Thus part (a) of the proposition follows immediately from the exact sequence (35) with $j=-2$. For $j \neq-2$, the exact sequence (35) implies that the inclusion-induced map

$$
\mathbb{Z}\left\{E_{\Lambda}, H_{\Lambda}\right\} \oplus \bigoplus_{j \neq-2} H_{*}^{(j)}(\Lambda \backslash c) \longrightarrow \bigoplus_{j \neq-2} H_{*}^{(j)}(\Lambda)
$$

is an isomorphism. Part (b) of the proposition follows from this and Lemma 5.8.

At this point it is not hard to compute the rest of $H_{*}(\Lambda)$, namely $H_{0}^{(-2)}(\Lambda)$, and also to take the direct limit over $\Lambda$ to recover $H_{*}(2 \pi ; 0)$ and $\widetilde{H}_{*}(2 \pi ; 0)$ as described in Section 1.3. (The notation $Z_{1}(c, b)$ here corresponds to $h(c, b)$ in Section 1.3.) We will do these calculations in greater generality in Section 6.2 and Section 7.3 respectively.

\section{Flattening and applications}

The previous section did most of the calculation of $\widetilde{H}_{*}(2 \pi n ; 0)$ when $n=1$, which entailed calculating most of $H_{*}(\Lambda)$ where $\Lambda$ is a closed admissible path of rotation number 1 . In general $H_{*}(\Lambda)$ is much more complicated when $\Lambda$ has rotation number $n>1$. To simplify the calculations for arbitrary $n$, in Section 6.1 we introduce a "flattening" technique, exemplified by Proposition 6.14 below, which reduces many homological calculations to more manageable ones involving admissible paths on the $x$-axis. As a first application, in Section 6.2 we compute $H_{0}^{(-2 n)}(\Lambda)$ where $\Lambda$ is $n$-convex, which means the following:

Definition 6.1 An $n$-convex path is a closed admissible path $\Lambda$ of rotation number $n$ which is the pullback, via the projection $\mathbb{R} / 2 \pi n \mathbb{Z} \rightarrow \mathbb{R} / 2 \pi \mathbb{Z}$, of a closed admissible path of rotation number 1. By Example 2.3, the latter corresponds to a convex polygonal region $P_{\Lambda}$ in $\mathbb{R}^{2}$ with corners in $\mathbb{Z}^{2}$, possibly a 2 -gon or a point. Let $k_{\Lambda}$ denote the number of lattice points in $P_{\Lambda}$.

As a second application of the flattening technology, we will prove in Section 6.3 that the homology vanishes for periodic paths that are not closed: 
Theorem 6.2 For any $n$, if $\Gamma \neq 0$, then

$$
H_{*}(2 \pi n ; \Gamma)=0 .
$$

Note that this is equivalent to $\widetilde{H}_{*}(2 \pi n ; \Gamma)=0$, see Section 3.1.4. By the universal coefficient spectral sequence (see Section 8.1), this implies Theorem 1.3(a).

\subsection{Flattening}

Let $\Lambda$ be a closed admissible path of rotation number $n$, and fix an angle $\theta \in(0,2 \pi)$ with $\tan \theta$ irrational. We now define a subcomplex $C_{*}^{\theta}(\Lambda)$ of $C_{*}(\Lambda)$ which is much smaller but has the same homology. The first step is to define a decomposition of the $\mathbb{Z}$-module $C_{*}(\Lambda)$ in terms of " $\theta$-corner sequences".

Definition 6.3 For $\lambda \leq \Lambda$, the $\theta$-corner sequence associated to $\lambda$ is the sequence $\left(p_{0}, p_{1}, \ldots, p_{2 n}=p_{0}\right)$ of points in $\mathbb{Z}^{2}$ defined by

$$
p_{i}:=\lambda(\theta+i \pi) .
$$

Let $S^{\theta}(\Lambda)$ denote the set of all $\theta$-corner sequences that can arise for admissible paths $\lambda \leq \Lambda$. The set $S^{\theta}(\Lambda)$ can be characterized as follows. Define an ordering on $\mathbb{Z}^{2}$ by

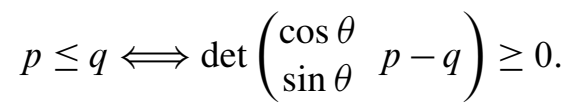

If $p \leq q$ and $i$ is odd, or $p \geq q$ and $i$ is even, let $s_{p, q}^{i}$ denote the open admissible path parametrized by the interval

$$
I_{i}:=(\theta+i \pi, \theta+(i+1) \pi)
$$

with endpoints at $p$ and $q$ and with a single edge if $p \neq q$ and no edges otherwise. If $\lambda^{\prime}$ and $\lambda$ are open admissible paths parametrized by the same interval, but (unlike in Definition 2.6) not necessarily having the same endpoints, write

$$
\lambda^{\prime} \leq^{\prime} \lambda
$$

if the inequality (11) holds.

Lemma 6.4 $\left(p_{0}, p_{1}, \ldots, p_{2 n}=p_{0}\right) \in S^{\theta}(\Lambda)$ if and only if for each $i$, letting $p:=p_{i}$ and $q:=p_{i+1}$, we have

(i) $p \geq q$ if $i$ is even, and $p \leq q$ if $i$ is odd.

(ii) $s_{p, q}^{i} \leq\left.^{\prime} \Lambda\right|_{I_{i}}$. 
Proof $(\Rightarrow)$ If $\left(p_{0}, \ldots, p_{2 n}\right)$ is the $\theta$-corner sequence associated to $\lambda \leq \Lambda$, then condition (i) holds since $\lambda$ is an admissible path, and condition (ii) holds because $s_{p_{i}, p_{i+1}}^{i} \leq\left.\lambda\right|_{I_{i}} \leq\left.^{\prime} \Lambda\right|_{I_{i}} .(\Leftarrow)$ If $\left(p_{0}, \ldots, p_{2 n}\right)$ satisfies conditions (i) and (ii), then $\lambda=$ $s_{p_{0}, p_{1}}^{0} \cdots s_{p_{2 n-1}, p_{2 n}}^{2 n-1}$ is well defined by (i), satisfies $\lambda \leq \Lambda$ by (ii), and has $\left(p_{0}, \ldots, p_{2 n}\right)$ as its $\theta$-corner sequence.

For a given $i$, if $p$ and $q$ satisfy conditions (i) and (ii) above, then there is a unique maximal open admissible path $\Lambda_{p, q}^{i}$ parametrized by $I_{i}$ with endpoints at $p$ and $q$ and with $\Lambda_{p, q}^{i} \leq\left.^{\prime} \Lambda\right|_{I_{i}}$. The path $\Lambda_{p, q}^{i}$ traverses part of the boundary of the convex hull of the set of lattice points enclosed by $s_{p, q}^{i},\left.\Lambda\right|_{I_{i}}$, and rays from $p$ and from $q$ in the direction

$$
\left(\begin{array}{c}
\cos \theta \\
\sin \theta
\end{array}\right) \quad \text { if } i \text { is even, and } \quad\left(\begin{array}{l}
-\cos \theta \\
-\sin \theta
\end{array}\right) \text { if } i \text { is odd. }
$$

Example 6.5 The picture below shows an example where $n=1$ and $\theta$ is slightly greater than zero. Here the outer polygon is $\Lambda$, the numbers indicate the ordering (36) of the 10 lattice points in $P_{\Lambda}$, and the inner path is $\Lambda_{8,3}^{0}$.

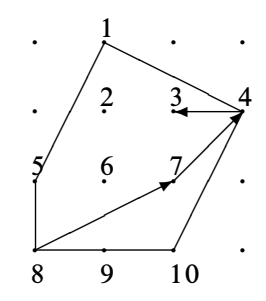

Lemma 6.6 As $\mathbb{Z}$-modules,

$$
C_{*}(\Lambda)=\bigoplus_{\left(p_{0}, \ldots, p_{2 n}\right) \in S^{\theta}(\Lambda)} \bigotimes_{i=0}^{2 n-1} C_{*}\left(\Lambda_{p_{i}, p_{i+1}}^{i}\right) .
$$

Proof It is enough to show that cyclic concatenation induces a bijection

$$
\{\lambda \leq \Lambda\}=\bigsqcup_{\left(p_{0}, \ldots, p_{2 n}\right) \in S^{\theta}(\Lambda)} \prod_{i=0}^{2 n-1}\left\{\lambda_{i} \leq \Lambda_{p_{i}, p_{i+1}}^{i}\right\} .
$$

If $\left(p_{0}, \ldots, p_{2 n}\right) \in S^{\theta}(\Lambda)$ and $\lambda_{i} \leq \Lambda_{p_{i}, p_{i+1}}^{i}$ for $i=0, \ldots, 2 n-1$, then the cyclic concatenation $\lambda=\lambda_{0} \lambda_{1} \cdots \lambda_{2 n-1}$ satisfies $\lambda \leq \Lambda$ and has $\left(p_{0}, \ldots, p_{2 n}\right)$ as its $\theta-$ corner sequence. Conversely, any $\lambda \leq \Lambda$ is obtained this way, where $\left(p_{0}, \ldots, p_{2 n}\right)$ is $\lambda$ 's $\theta$-corner sequence and $\lambda_{i}=\left.\lambda\right|_{I_{i}}$. 
Lemma 6.7 For a given $i$, suppose that the pairs $(p, q)$ and $\left(p^{\prime}, q^{\prime}\right)$ satisfy conditions (i) and (ii) above. Suppose that under the ordering (36), the interval between $p$ and $q$ contains the interval between $p^{\prime}$ and $q^{\prime}$. Then

$$
\Lambda_{p^{\prime}, q^{\prime}}^{i} \leq^{\prime} \Lambda_{p, q}^{i}
$$

Proof Since $\left.\Lambda\right|_{I_{i}}$ is parametrized by an interval of length $\pi$, we can find a convex polygonal region $P$ such that $\left.\Lambda\right|_{I_{i}}$ is part of the boundary of $P$. Then the right side of (38) is part of the boundary of the convex hull of the set of lattice points in $P$ that are between $p$ and $q$ in the ordering (36), inclusive. The left side of (38) has an analogous description for a subset of these lattice points. The relation (38) then follows as in Example 2.9.


Section 3.1.6. In terms of the decomposition (37), define

$$
C_{*}^{\theta}(\Lambda):=\bigoplus_{\left(p_{0}, \ldots, p_{2 n}\right) \in S^{\theta}(\Lambda)} \bigotimes_{i=0}^{2 n-1} \operatorname{span}\left\{E_{p_{i}, p_{i}+1}^{i}, H_{p_{i}, p_{i+1}}^{i}\right\} \subset C_{*}(\Lambda) .
$$

Lemma 6.9 $C_{*}^{\theta}(\Lambda)$ is a subcomplex of $C_{*}(\Lambda)$.

Proof For $\left(p_{0}, \ldots, p_{2 n}\right) \in S^{\theta}(\Lambda)$, define the cyclic concatenation

$$
\Lambda\left(p_{0}, p_{1}, \ldots, p_{2 n}\right):=\Lambda_{p_{0}, p_{1}}^{0} \cdots \Lambda_{p_{2 n-1}, p_{2 n}}^{2 n-1} .
$$

Recall from Section 4 that $c_{\theta+i \pi}$ denotes the corner of $\Lambda$ containing $\theta+i \pi$. By Lemma 3.8, it is enough to show that if $\left(p_{0}, \ldots, p_{2 n}\right) \in S^{\theta}(\Lambda)$ and $c_{\theta+i \pi}$ is not a kink of $\Lambda\left(p_{0}, p_{1}, \ldots, p_{2 n}\right)$, then

$$
\Lambda\left(p_{0}, \ldots, p_{2 n}\right) \backslash c_{\theta+i \pi}=\Lambda\left(p_{0}, \ldots, p_{i-1}, p_{i}^{\prime}, p_{i+1}, \ldots, p_{2 n}\right)
$$

where

$$
p_{i}^{\prime}:=\left(\Lambda\left(p_{0}, \ldots, p_{2 n}\right) \backslash c_{\theta+i \pi}\right)(\theta+i \pi) .
$$

By locality of rounding it is enough to show that

$$
\Lambda_{p_{i-1}, p_{i}}^{i-1} \Lambda_{p_{i}, p_{i+1}}^{i} \backslash c_{\theta+i \pi}=\Lambda_{p_{i-1}, p_{i}^{\prime}}^{i-1} \Lambda_{p_{i}^{\prime}, p_{i+1}}^{i} .
$$

In (39) we have $\leq$ by definition, and $\geq$ by Proposition 2.10(b) and Lemma 6.7, hence $=$ by Proposition 2.8 .

Example 6.10 If $\Lambda_{0}$ is on the $x$-axis, then $C_{*}^{\theta}\left(\Lambda_{0}\right)=C_{*}\left(\Lambda_{0}\right)$. Here $E_{p, q}^{i}$ and $H_{p, q}^{i}$ are single edges labeled ' $e$ ' and ' $h$ ' respectively (when $p \neq q$ ). 
Proposition 6.11 For any closed admissible path $\Lambda$ of rotation number $n$, the inclusion $C_{*}^{\theta}(\Lambda) \rightarrow C_{*}(\Lambda)$ induces an isomorphism on homology.

Proof By Proposition 2.13 and induction on length, there are only finitely many admissible paths to the left of $\Lambda$. Hence only finitely many points in $\mathbb{Z}^{2}$ can appear in a sequence in $S^{\theta}(\Lambda)$; denote these by $q_{1}, \ldots, q_{k}$, in increasing order with respect to the ordering (36). If $\alpha$ is a generator of $C_{*}(\Lambda)$ with $\theta$-corner sequence $\left(q_{j_{0}}, \ldots, q_{j_{2 n}}\right) \in$ $S^{\theta}(\Lambda)$, define the "degree"

$$
\operatorname{deg}(\alpha):=\sum_{i=0}^{2 n-1}(-1)^{i} j_{i} \geq 0 .
$$

This defines an increasing filtration on $C_{*}(\Lambda)$. Indeed the differential $\delta$ on $C_{*}(\Lambda)$ splits as

$$
\delta=\delta_{0}+\delta_{1}
$$

where $\delta_{1}$ is the contribution from rounding at the corners $c_{\theta+i \pi}$; then $\delta_{0}$ preserves the degree while $\delta_{1}$ decreases it (possibly by an arbitrarily large amount). This filtration yields a spectral sequence $E_{*, *}^{*}$ converging to $H_{*}(\Lambda)$. The $E^{1}$ term is the homology of $\delta_{0}$. By equation (28), the differential $\delta_{0}$ agrees with the tensor product differential on the right hand side of (37), with the standard sign if the factors in the tensor product are arranged in the order $i=2 n-1, \ldots, 0$. So by Proposition 5.6, the inclusion $C_{*}^{\theta}(\Lambda) \rightarrow C_{*}(\Lambda)$ induces an isomorphism

$$
C_{*}^{\theta}(\Lambda)=E^{1} .
$$

We can filter the subcomplex $C_{*}^{\theta}(\Lambda)$ the same way to obtain a spectral sequence ' $E_{*, *}^{*}$ converging to the homology of $C_{*}^{\theta}(\Lambda)$. Now $\delta_{0}$ restricts to zero on $C_{*}^{\theta}(\Lambda)$ by Lemma 3.7 , so

$$
{ }^{\prime} E^{1}=C_{*}^{\theta}(\Lambda)
$$

Putting this together, the inclusion of filtered complexes $C_{*}^{\theta}(\Lambda) \rightarrow C_{*}(\Lambda)$ induces a morphism of spectral sequences, which by equations (40) and (41) induces an isomorphism ' $E^{1} \simeq E^{1}$. Hence the inclusion induces an isomorphism ' $E^{\infty} \simeq E^{\infty}$, and therefore an isomorphism on homology.

We now specialize to the case where $\Lambda$ is $n$-convex.

Lemma 6.12 Let $\Lambda, \Lambda^{\prime}$ be $n$-convex paths with $k_{\Lambda}=k_{\Lambda^{\prime}}$. Then there is a canonical isomorphism of $\mathbb{Z}$-graded chain complexes

$$
C_{*}^{\theta}(\Lambda)=C_{*}^{\theta}\left(\Lambda^{\prime}\right)
$$


Proof We compute the chain complex $C_{*}^{\theta}(\Lambda)$ explicitly. Denote the lattice points enclosed by $\Lambda$ in increasing order with respect to the ordering (36) by $1, \ldots, k$. By Lemma $6.4,\left(j_{0}, \ldots, j_{2 n}\right) \in S^{\theta}(\Lambda)$ if and only if $j_{i} \geq j_{i+1}$ for $i$ even and $j_{i} \leq j_{i+1}$ for $i$ odd. Moreover if $i$ is even and $j, j^{\prime \prime}>j^{\prime}$, then $c_{\theta+i \pi}$ is not a kink of $\Lambda_{j, j^{\prime}}^{i-1} \Lambda_{j^{\prime}, j^{\prime \prime}}^{i}$, and

$$
\Lambda_{j, j^{\prime}}^{i-1} \Lambda_{j^{\prime}, j^{\prime \prime}}^{i} \backslash c_{\theta+i \pi}=\Lambda_{j, j^{\prime}+1}^{i-1} \Lambda_{j^{\prime}+1, j^{\prime \prime}}^{i}
$$

Here $\geq$ holds and $c_{\theta+i \pi}$ is not a kink by Lemma 6.7 and Propositions 2.10(b) and 2.12, while $\leq$ holds by equation (39) and Lemma 6.7. Likewise, if $i$ is odd and $j, j^{\prime \prime}<j^{\prime}$ then

$$
\Lambda_{j, j^{\prime}}^{i-1} \Lambda_{j^{\prime}, j^{\prime \prime}}^{i} \backslash c_{\theta+i \pi}=\Lambda_{j, j^{\prime}-1}^{i-1} \Lambda_{j^{\prime}-1, j^{\prime \prime}}^{i}
$$

So by Lemma 3.8, the differential on $C_{*}^{\theta}(\Lambda)$ operates on a length $2 n$ cyclic string of $E$ 's and $H$ 's according to the local (up to sign) rules

$$
\begin{aligned}
& E_{j, j^{\prime}}^{i-1} H_{j^{\prime}, j^{\prime \prime}}^{i}, H_{j, j^{\prime}}^{i-1} E_{j, j^{\prime \prime}}^{i} \longmapsto \pm E_{j, j^{\prime}+1}^{i-1} E_{j^{\prime}+1, j^{\prime \prime}}^{i}, \\
& H_{j, j^{\prime}}^{i-1} H_{j^{\prime}, j^{\prime \prime}}^{i} \longmapsto \pm\left(E_{j, j^{\prime}+1}^{i-1} H_{j^{\prime}+1, j^{\prime \prime}}^{i}+H_{j, j^{\prime}+1}^{i-1} E_{j^{\prime}+1, j^{\prime \prime}}^{i}\right),
\end{aligned}
$$

for $i$ even and $j, j^{\prime \prime}>j^{\prime}$, and similarly for $i$ odd.

The important point is that the above description of the chain complex $C_{*}^{\theta}(\Lambda)$ depends only on $k$. Thus we get a canonical isomorphism of chain complexes $C_{*}^{\theta}(\Lambda)=C_{*}^{\theta}\left(\Lambda^{\prime}\right)$. This isomorphism respects the grading, as one can see by using rounding and relabeling to inductively reduce to generators involving constant corner sequences.

Definition 6.13 Let $\Lambda, \Lambda_{0}$ be $n$-convex paths with $k_{\Lambda}=k_{\Lambda_{0}}$ and with $P_{\Lambda_{0}}$ on the $x$-axis. Define a chain map

$$
F_{\theta}: C_{*}\left(\Lambda_{0}\right) \longrightarrow C_{*}(\Lambda)
$$

to be the composition of canonical isomorphisms and inclusion

$$
C_{*}\left(\Lambda_{0}\right)=C_{*}^{\theta}\left(\Lambda_{0}\right)=C_{*}^{\theta}(\Lambda) \longrightarrow C_{*}(\Lambda) .
$$

Explicitly, $F_{\theta}$ takes a generator of $C_{*}\left(\Lambda_{0}\right)$ and replaces each ' $e$ ' or ' $h$ ' edge by a corresponding $E_{p, q}^{i}$ or $H_{p, q}^{i}$ to obtain a cyclic concatenation of such in $C_{*}^{\theta}(\Lambda) \subset$ $C_{*}(\Lambda)$.

Proposition 6.14 Let $\Lambda, \Lambda_{0}$ be $n$-convex paths with $k_{\Lambda}=k_{\Lambda_{0}}$ and with $P_{\Lambda_{0}}$ on the $x$-axis. Then:

(a) The chain map $F_{\theta}: C_{*}\left(\Lambda_{0}\right) \rightarrow C_{*}(\Lambda)$ induces an isomorphism on homology

$$
\left(F_{\theta}\right)_{*}: H_{i}^{(j)}\left(\Lambda_{0}\right) \stackrel{\simeq}{\longrightarrow} H_{i}^{(j)}(\Lambda) .
$$


(b) Let $\Lambda^{\prime}$ and $\Lambda_{0}^{\prime}$ be the $n$-convex paths obtained by rounding the distinguished corners $c_{\theta}$ of $P_{\Lambda}$ and $P_{\Lambda_{0}}$ respectively. Then the diagram

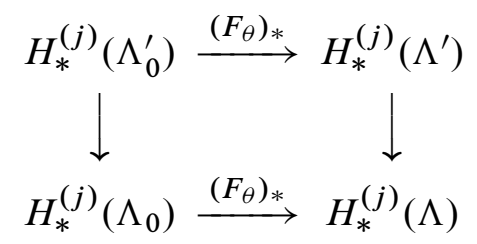

commutes, where the vertical arrows are induced by inclusion.

Proof Part (a) follows from Proposition 6.11; the upper index $j$ is preserved because $F_{\theta}$ preserves the number of ' $h$ ' edges. The diagram in part (b) commutes at the chain level, because if $q_{k}$ denotes the maximal lattice point in $P_{\Lambda}$ with respect to the ordering (36), then $C_{*}^{\theta}\left(\Lambda^{\prime}\right)$ is the subcomplex of $C_{*}^{\theta}(\Lambda)$ in which $q_{k}$ does not appear in any of the $\theta$-corner sequences.

\subsection{The special degree zero homology}

We now apply Proposition 6.14 to compute $H_{0}^{(-2 n)}(\Lambda)$, where $\Lambda$ is an $n$-convex path. Generators for this homology are given explicitly as follows.

Definition 6.15 If $a, b \in P_{\Lambda}$ are distinct lattice points with $b-a$ indivisible, define $Z_{n}(a, b) \in C_{*}(\Lambda)$ to be the generator that wraps $n$ times around the 2-gon with vertices $a$ and $b$, with all $2 n$ edges labeled ' $h$ ', ordered counterclockwise with an edge from $a$ to $b$ coming first. If $a, b \in P_{\Lambda}$ are distinct lattice points with $m-1$ lattice points in the interior of the line segment between them, define

$$
Z_{n}(a, b):=\sum_{i=1}^{m} Z_{n}\left(a+\frac{i-1}{m}(b-a), a+\frac{i}{m}(b-a)\right) .
$$

Define $Z_{n}(a, a):=0$. Observe that $\delta Z_{n}(a, b)=0$.

Definition 6.16 A simple triangle is a triple $(a, b, c)$ of non-colinear points in $\mathbb{Z}^{2}$, ordered counterclockwise, such that the triangle with vertices $a, b, c$ encloses no other lattice points.

Lemma 6.17 Let $\Lambda$ be an $n$-convex path. Then for any lattice points $a, b, c \in P_{\Lambda}$, the following relations hold in the homology $H_{*}(\Lambda)$ :

$$
\begin{gathered}
Z_{n}(a, b)+Z_{n}(b, a)=0, \\
Z_{n}(a, b)+Z_{n}(b, c)+Z_{n}(c, a)=0 .
\end{gathered}
$$


Proof Equation (43) holds at the chain level because $Z_{n}(b, a)$ is obtained from $Z_{n}(a, b)$ by re-ordering the ' $h$ ' edges, and a $2 n-$ cycle is an odd permutation.

Likewise, equation (44) holds at the chain level if $a, b, c$ are colinear.

Next we prove (44) when $(a, b, c)$ is a simple triangle. Let $\lambda$ be the $n$-convex path that wraps $n$ times around this triangle. Write the corners of $\lambda$ in counterclockwise order as $c_{1}, \ldots, c_{3 n}$, starting with a corner that maps to $a$. Define an "admissible corner set" to be a subset $I \subset\{1, \ldots, 3 n\}$ such that for distinct $i, j \in I$, the corners $c_{i}, c_{j}$ are not adjacent, ie $|i-j| \neq 1,3 n-1$. Let $\mathcal{C}$ denote the set of admissible corner sets. If $I=\left\{i_{1}, \ldots, i_{k}\right\} \in \mathcal{C}$, let

$$
\lambda(I):=\lambda \backslash c_{i_{1}} \backslash c_{i_{2}} \backslash \cdots \backslash c_{i_{k}} .
$$

Let $T(I) \in C_{*}(\Lambda)$ denote the generator with underlying path $\lambda(I)$ and with all edges labeled ' $h$ '. Order the ' $h$ ' edges counterclockwise, starting at $c_{1}$ if $1 \notin I$ and starting at $c_{2}$ if $1 \in I$. In this notation,

$$
\begin{aligned}
& Z_{n}(a, b)=T(\{3,6, \ldots, 3 n\}), \\
& Z_{n}(b, c)=T(\{1,4, \ldots, 3 n-2\}), \\
& Z_{n}(c, a)=-T(\{2,5, \ldots, 3 n-1\}) .
\end{aligned}
$$

The differential of a generator $T(I)$ is given by

$$
\delta T(I)=\sum_{I \cup\{i\} \in \mathcal{C}}(-1)^{\#\{j \notin I \mid i<j\}} T(I \cup\{i\}) .
$$

Now let $\mathcal{C}_{0}$ denote the set of admissible corner sets of the form $I=\left\{i_{1}, \ldots, i_{n-1}\right\}$ with $i_{1}<i_{2}<\cdots<i_{n-1}$ and with $i_{1}, i_{3}, \ldots$ odd and $i_{2}, i_{4}, \ldots$ even. By (45) and (46),

$$
\delta\left(\sum_{I \in \mathcal{C}_{0}} T(I)\right)=Z_{n}(a, b)+Z_{n}(b, c)+Z_{n}(c, a) .
$$

To see this, observe that a generator $T(I)$ with $I=\left\{i_{1}<i_{2}<\cdots<i_{n}\right\}$ will appear exactly once in the left hand side if the $i_{j}$ 's alternate parity, twice with opposite signs if there is exactly one $j$ such that $i_{j}$ and $i_{j+1}$ have the same parity, and otherwise not at all.

For general noncolinear lattice points $a, b, c \in P_{\Lambda}$, we can triangulate the triangle with vertices $a, b, c$ by simple triangles. Adding the relations (44) for these simple triangles, and using (43) to cancel interior edges, proves (44) for $(a, b, c)$.

The following is a useful way to understand the above relations. If $S$ is any set, let $\mathcal{I}(S)$ denote the set of finite formal sums of elements of $S$ with integer coefficients such 
that the sum of the coefficients is zero. For $a, b \in S$, define $z(a, b):=a-b \in \mathcal{I}(S)$. We then have the following elementary fact, whose proof is left to the reader:

Lemma 6.18 If $S$ is any set, then as a $\mathbb{Z}$-module, $\mathcal{I}(S)$ is generated by

$$
\{z(a, b) \mid a, b \in S\},
$$

with the relations $z(a, b)+z(b, a)=0$ and $z(a, b)+z(b, c)+z(c, a)=0$.

Proposition 6.19 Let $\Lambda$ be an n-convex path. Then there is an isomorphism

$$
\mathcal{I}\left(P_{\Lambda} \cap \mathbb{Z}^{2}\right) \simeq H_{0}^{(-2 n)}(\Lambda)
$$

sending $z(a, b) \mapsto Z_{n}(a, b)$.

Proof By Lemmas 6.17 and 6.18, the above map $\mathcal{I}\left(P_{\Lambda} \cap \mathbb{Z}^{2}\right) \rightarrow H_{0}^{(-2 n)}$ is welldefined. To show that it is an isomorphism, let $k=k_{\Lambda}$ and let $\Lambda_{0}$ be the $n$-convex path on the $x$-axis with $P_{\Lambda_{0}}=[1, k] \times\{0\}$. Choose any $\theta \in(0, \pi)$ with $\tan \theta$ irrational. By Proposition 6.14(a), there is an isomorphism on homology

$$
\left(F_{\theta}\right)_{*}: H_{*}^{(j)}\left(\Lambda_{0}\right) \stackrel{\simeq}{\longrightarrow} H_{*}^{(j)}(\Lambda) .
$$

Since every generator of $C_{*}\left(\Lambda_{0}\right)$ has nonnegative index (cf equation (49) below) and at most $2 n$ edges labeled ' $h$ ', the generators of $C_{*}^{(-2 n)}\left(\Lambda_{0}\right)$ are those with $2 n$ edges labeled ' $h$ ' and with index 0 , which since all edges are labeled ' $h$ ' means that all edges have length one. Thus $C_{*}^{(-2 n)}\left(\Lambda_{0}\right)=H_{0}^{(-2 n)}\left(\Lambda_{0}\right)$ is freely generated by the $k-1$ generators $Z_{n}((1,0),(2,0)), \ldots, Z_{n}((k-1,0),(k, 0))$.

By the construction of the chain map $F_{\theta}$ in Section 6.1,

$$
F_{\theta}\left(Z_{n}((i, 0),(i+1,0))\right)=Z_{n}\left(q_{i}, q_{i+1}\right)
$$

where $q_{1}, \ldots, q_{k}$ are the lattice points in $P_{\Lambda}$, ordered by (36). Hence $H_{0}^{(-2 n)}(\Lambda)$ is freely generated by $Z_{n}\left(q_{1}, q_{2}\right), \ldots, Z_{n}\left(q_{k-1}, q_{k}\right)$. But it follows from the definition of $\mathcal{I}$ that $\mathcal{I}\left(P_{\Lambda} \cap \mathbb{Z}^{2}\right)$ is freely generated by $z\left(q_{1}, q_{2}\right), \ldots, z\left(q_{k-1}, q_{k}\right)$.

\subsection{Vanishing of homology for $\Gamma \neq 0$}

We now prove Theorem 6.2. Without loss of generality,

$$
\Gamma=\left(\begin{array}{c}
k \\
0
\end{array}\right)
$$


for some positive integer $k$. This is justified by the following lemma. An element $A \in S L_{2} \mathbb{Z}$ induces a diffeomorphism $S^{1} \rightarrow S^{1}$, where $S^{1}$ is the unit circle in the $\mathbb{R}^{2}$ on which $A$ acts. This diffeomorphism can be lifted to a diffeomorphism $f: \mathbb{R} \rightarrow \mathbb{R}$. The set of pairs $(A, f)$ forms a group $\widetilde{S L}_{2} \mathbb{Z}$ which is an infinite cyclic cover of $S L_{2} \mathbb{Z}$.

Lemma 6.20 A pair $(A, f) \in \widetilde{S L}_{2} \mathbb{Z}$ induces an isomorphism

$$
\Phi_{(A, f)}: H_{*}(2 \pi n ; \Gamma) \stackrel{\simeq}{\longrightarrow} H_{*}(2 \pi n ; A \Gamma) .
$$

Proof If $\Lambda: \mathbb{R} \backslash p^{-1}(T) \rightarrow \mathbb{Z}^{2}$ is an admissible path of rotation number $n$ and period $\Gamma$, define an admissible path $\Phi \Lambda$ of period $A \Gamma$ by

$$
\Phi \Lambda:=A \circ \Lambda \circ f^{-1} .
$$

Then $f$ induces a bijection from the edges of $\Lambda$ to the edges of $\Phi \Lambda$, and pushing forward edge labels via this bijection gives an isomorphism of $\mathbb{Z}$-modules

$$
\Phi_{(A, f)}: C_{*}(2 \pi n ; \Gamma) \longrightarrow C_{*}(2 \pi n ; A \Gamma) .
$$

It follows from the definition of rounding that this is a chain map, since the action of $S L_{2} \mathbb{Z}$ on $\mathbb{Z}^{2}$ preserves convex hulls and (unlike the more general action of $G L_{2} \mathbb{Z}$ ) respects the signs in the differential $\delta$.

For $a, b \geq 1$, let $\square(a, b ; k)$ denote the closed admissible path of rotation number $n$ and period $\Gamma$ whose restriction to $(0,2 \pi n]$ wraps $n$ times around the rectangle $[0, a-1] \times[0, b-1] \subset \mathbb{R}^{2}$, except that the edge at $\theta=2 \pi n$ has length $a-1+k$ For example, $\square(1,1 ; k)$ has edges of length $k$ along the $x$-axis at angles $\theta=2 \pi i n$ separated by kinks parametrized by the intervals $(2 \pi i n, 2 \pi(i+1) n)$. We now have the following analogue of Proposition 6.14(a), which replaces the path $\square(a, b ; k)$ by a path on the $x$-axis.

Lemma 6.21 There is a chain map

$$
F: C_{*}(\square(a b, 1 ; k b)) \longrightarrow C_{*}(\square(a, b ; k))
$$

which induces an isomorphism on homology, preserves the relative grading, and sends

$$
E_{\square(1,1 ; k b)} \longmapsto E_{\square(1,1 ; k)} .
$$

Proof If $\Lambda$ is any periodic admissible path of rotation number $n$ and period $\Gamma$, and if $\theta \in(0,2 \pi)$ is an angle with $\tan \theta$ irrational, then we can define a subcomplex $C_{*}^{\theta}(\Lambda) \subset C_{*}(\Lambda)$ as in Section 6.1. The only difference is that now a $\theta$-corner sequence 
is an infinite sequence $\left\{p_{i} \mid i \in \mathbb{Z}\right\}$ of points in $\mathbb{Z}^{2}$ such that $p_{i+2 n}=p_{i}+\Gamma$ for all $i$. As in Proposition 6.11, the inclusion $C_{*}^{\theta}(\Lambda) \rightarrow C_{*}(\Lambda)$ induces an isomorphism on homology.

Now let $\theta:=\frac{\pi}{2}+\epsilon$ where $\epsilon>0$ is small with respect to $b$. Then for two consecutive points in a $\theta$-corner sequence for $\square(a, b ; k)$, the ordering (36) coincides with the lexicographic order on $\mathbb{Z}^{2}$. There is then an isomorphism of chain complexes

$$
C_{*}^{\theta}(\square(a, b ; k)) \stackrel{\simeq}{\longrightarrow} C_{*}^{\theta}(\square(a b, 1 ; k b))=C_{*}(\square(a b, 1 ; k b)) .
$$

This is defined via the bijection on $\theta$-corner sequences induced by the map

$$
\begin{aligned}
\mathbb{Z} \times\{0,1, \ldots, b-1\} & \longrightarrow \mathbb{Z} \times\{0\}, \\
(x, y) & \longmapsto(b x+y, 0) .
\end{aligned}
$$

This is a chain map and preserves the relative grading as in the proof of Lemma 6.12, because for $\Lambda=\square(a, b ; k)$, locally $\Lambda_{p_{i-1}, p_{i}}^{i-1}$ and $\Lambda_{p_{i}, p_{i+1}}^{i}$ are the same as they would be if $\Lambda$ were $n$-convex and $P_{\Lambda}$ were a rectangle. Clearly this isomorphism sends $E_{\square(1,1 ; k)}$ to $E_{\square(1,1 ; k b)}$.

The homology $H_{*}(\square(a, b ; k))$ may be complicated, but we will only need to establish a lower bound on the index of nonvanishing homology groups. For $y_{0} \in \mathbb{Z}$, let $C_{*}\left(2 \pi n ; \Gamma ; y \geq y_{0}\right)$ denote the subcomplex of $C_{*}(2 \pi n ; \Gamma)$ spanned by generators whose underlying admissible paths map to the half-plane $y \geq y_{0}$.

Lemma 6.22 If $\alpha \in H_{*}(2 \pi n ; \Gamma ; y \geq 0)$ is nonzero and has pure degree, then the relative index

$$
I\left(\alpha, E_{\square(1,1 ; k)}\right) \geq-n .
$$

Proof By horizontally translating a cycle representing $\alpha$ (which does not affect the index by (18)), we may assume that $\alpha$ is contained in $H_{*}(\square(a, b ; k))$ for some $a, b$. By Lemma 6.21, $\alpha=F\left(\alpha_{0}\right)$ for some $\alpha_{0} \in H_{*}(\square(a b ; 1 ; k b))$, and

$$
I\left(\alpha, E_{\square(1,1 ; k)}\right)=I\left(\alpha_{0}, E_{\square(1,1 ; k b)}\right) .
$$

Let $\beta_{0}$ be a generator in a cycle representing $\alpha_{0}$. Since $\beta_{0}$ and $E_{\square(1,1 ; k b)}$ are on the $x$-axis, the definition of the relative index (16) implies that

$$
I\left(\beta_{0}, E_{\square(1,1 ; k b)}\right)=\ell\left(\beta_{0}\right)-\# h\left(\beta_{0}\right)-k b .
$$

Since $\beta_{0}$ has period

$$
\left(\begin{array}{c}
k b \\
0
\end{array}\right)
$$

we see that $\ell\left(\beta_{0}\right)-\# h\left(\beta_{0}\right) \geq k b-n$. 
Proof of Theorem 6.2 Suppose $\alpha \in H_{*}(2 \pi n ; \Gamma)$ is nonzero and has pure degree. Since

$$
C_{*}(2 \pi n ; \Gamma)=\lim _{y_{0} \rightarrow-\infty} C_{*}\left(2 \pi n ; \Gamma ; y \geq y_{0}\right),
$$

there exists $y_{0}$ such that

$$
\alpha \in H_{*}\left(2 \pi n ; \Gamma ; y \geq y_{0}-m\right)
$$

for all $m \geq 0$. Let $\Psi: C_{*}(2 \pi n ; \Gamma) \rightarrow C_{*}(2 \pi n ; \Gamma)$ be the isomorphism of chain complexes that translates paths upward by one unit, ie

$$
(\Psi \Lambda)(t):=\Lambda(t)+\left(\begin{array}{l}
0 \\
1
\end{array}\right)
$$

By Lemma 6.22 and symmetry, for all $m \geq 0$,

$$
I\left(\alpha, \Psi^{y_{0}-m} E_{\square(1,1 ; k)}\right) \geq-n .
$$

By the index ambiguity formula (18),

$$
\begin{aligned}
I\left(\alpha, \Psi^{y_{0}-m} E_{\square(1,1 ; k)}\right) & =I\left(\alpha, \Psi^{y_{0}} E_{\square(1,1 ; k)}\right)+I\left(\Psi^{y_{0}} E_{\square(1,1 ; k)}, \Psi^{y_{0}-m} E_{\square(1,1 ; k)}\right) \\
& =I\left(\alpha, \Psi^{y_{0}} E_{\square(1,1 ; k)}\right)-2 k m .
\end{aligned}
$$

Combining this with (47) gives a contradiction when $m$ is sufficiently large.

\section{Computation of $\tilde{H}_{*}(2 \pi n ; 0)$}

We now compute the homology for closed admissible paths of rotation number $n$. Recall the notations $\mathcal{I}\left(\mathbb{Z}^{2}\right)$ and $\mathbb{Z}$ from Section 1.3.5. Also recall the notation $\widetilde{H}_{*}^{(j)}(2 \pi n ; 0)$ from Definition 5.1. In this section we will prove:

Theorem 7.1 (a) As $\mathbb{Z}\left[\mathbb{Z}^{2}\right]-$ modules,

$$
\widetilde{H}_{i}^{(j)}(2 \pi n ; 0) \simeq\left\{\begin{array}{cl}
\underline{\mathbb{Z}}, & i=2 k, 2 k+1 ; j=2 k-2 n+2 ; k \in \mathbb{Z}_{\geq 0}, \\
\mathcal{I}\left(\mathbb{Z}^{2}\right), & i=0, j=-2 n, \\
0, & \text { otherwise. }
\end{array}\right.
$$

(b) If $i \geq 2$, then the map

$$
U: \widetilde{H}_{i}(2 \pi n ; 0) \longrightarrow \widetilde{H}_{i-2}(2 \pi n ; 0)
$$

is an isomorphism between the $\mathbb{Z}$ components. 
In particular, $\widetilde{H}_{*}(2 \pi n ; 0)$ is independent of $n$. However, the homology generators look very different for different $n$. Among other things, increasing $n$ by 1 increases the number of ' $h$ ' edges (in the chain complex generators that are summands in cycles generating the homology) by 2 . For example, if $\Lambda$ is a closed admissible path of rotation number $n$, then the cycles $E_{\Lambda}, H_{\Lambda} \in \widetilde{C}_{*}(2 \pi n ; 0)$ are homology generators when $n=1$, but they are boundaries when $n>1$.

\subsection{Splicing}

Definition 7.2 For $n \geq 1$, let $C X_{*}(n)$ denote the subcomplex of $C_{*}(2 \pi n ; 0)$ spanned by generators with underlying admissible path on the $x$-axis in $\mathbb{R}^{2}$. Let

$$
C X_{*}^{(j)}(n):=C X_{*}(n) \cap C_{*}^{(j)}(2 \pi n ; 0) .
$$

Thanks to the "flattening" technology of Section 6.1, to prove Theorem 7.1 it is mostly sufficient to compute the homology $H X_{*}(n)$. We will now compute $H X_{*}(n)$ by induction on $n$, using a "splicing" isomorphism constructed below.

We will use the following notation for generators of $C X_{*}(n)$. Similarly to Section 6.1 , define a corner sequence to be a sequence of integers $a_{0}, a_{1}, \ldots, a_{2 n}=a_{0}$ with $a_{0} \geq a_{1} \leq a_{2} \geq \cdots$, and let $\mathcal{S}_{n}$ denote the set of all corner sequences. If $\alpha$ is a generator of $C X_{*}(n)$, then the $x$-coordinates of the values of its underlying admissible path at $\pi / 2+i \pi$ determine a corner sequence $\left\{a_{i}\right\}$. Thus as a $\mathbb{Z}$-module,

$$
C X_{*}(n)=\bigoplus_{\left\{a_{i}\right\} \in \mathcal{S}_{n}} \bigotimes_{i=0}^{2 n-1} \mathbb{Z}\left\{e_{a_{i}}^{a_{i+1}}, h_{a_{i}}^{a_{i+1}}\right\} .
$$

Here $e_{a}^{b}$ and $h_{a}^{b}$ denote edges from $(a, 0)$ to $(b, 0)$ labeled ' $e$ ' and ' $h$ ' respectively. If $a=b$, then $e_{a}^{b}$ denotes the lack of an edge, and we interpret $h_{a}^{b}=0$. (So if $a=b$, then $\mathbb{Z}\left\{e_{a}^{b}, h_{a}^{b}\right\}$ really means $\mathbb{Z}\left\{e_{a}^{b}\right\}$.) This decomposition of $C X_{*}(n)$ determines a natural convention for ordering the ' $h$ ' edges. By the index formula (15), the index of a generator is the sum of the lengths of the edges, minus the number of ' $h$ ' edges. That is, if $\alpha$ has corner sequence $\left\{a_{i}\right\}$, then

$$
I(\alpha)=\sum_{i=0}^{2 n-1}\left|a_{i}-a_{i+1}\right|-\# h(\alpha) .
$$

The differential on $C X_{*}(n)$ is given as in equation (42).

Definition 7.3 Define the "splicing map"

$$
S: C X_{*}^{(j)}(n) \longrightarrow C X_{*}^{(j-2)}(n+1)
$$


as follows. In terms of the decomposition (48), any generator of $C X_{*}(n)$ has the form $w \otimes e_{a}^{b} \otimes e_{b}^{c}, w \otimes e_{a}^{b} \otimes h_{b}^{c}, w \otimes h_{a}^{b} \otimes e_{b}^{c}$, or $w \otimes h_{a}^{b} \otimes h_{b}^{c}$, where $w$ is a tensor product of $2 n-2 e$ 's or $h$ 's, and $a \geq b \leq c$. Henceforth we will omit the tensor product symbol when writing generators of $C X_{*}(n)$ this way. Now $S$ sums over all ways of shortening the edges from $a$ to $b$ and from $b$ to $c$ and splicing in two ' $h$ ' edges between them so that the grading is preserved. Namely

$$
\begin{aligned}
& S\left(w e_{a}^{b} e_{b}^{c}\right):=\sum_{i=b}^{a} \sum_{j=b}^{c} w e_{a}^{i} h_{i}^{i+j-b+1} h_{i+j-b+1}^{j} e_{j}^{c}, \\
& S\left(w h_{a}^{b} e_{b}^{c}\right):=\sum_{i=b}^{a-1} \sum_{j=b}^{c} w h_{a}^{i} h_{i}^{i+j-b+1} h_{i+j-b+1}^{j} e_{j}^{c},
\end{aligned}
$$

and similarly for $S\left(w e_{a}^{b} h_{b}^{c}\right)$ and $S\left(w h_{a}^{b} h_{b}^{c}\right)$.

Lemma 7.4 $S$ is a degree 0 chain map.

Proof $S$ preserves the grading because

$(a-b)+(c-b)=(a-i)+([i+j-b+1]-i)+([i+j-b+1]-j)+(c-j)-2$.

We now check that $\delta S=S \delta$. Recall the notation $c_{\theta}$ from Section 4. Decompose the differential on $C X_{*}(n)$ as $\delta=\delta_{0}+\delta_{1}$ where $\delta_{1}$ is the contribution from rounding at the corner $c_{(2 n-1 / 2) \pi}$. Similarly decompose the differential on $C X_{*}(n+1)$ as $\delta=\delta_{0}+\delta_{1}$ where $\delta_{1}$ is the contribution from rounding at the corners $c_{(2 n-1 / 2) \pi}, c_{(2 n+1 / 2) \pi}$, and $c_{(2 n+3 / 2) \pi}$. Clearly $\delta_{0} S=S \delta_{0}$; the signs agree because the number of ' $h$ ' edges we are splicing in is always even. So we just have to check that $\delta_{1} S=S \delta_{1}$. We will verify that $\delta_{1} S \alpha=S \delta_{1} \alpha$ when $\alpha=w e_{a}^{b} h_{b}^{c}$; the calculations for the other three types of generators are very similar. By the definitions of $S$ and $\delta_{1}$,

$$
\begin{aligned}
\delta_{1} S\left(w e_{a}^{b} h_{b}^{c}\right) & =\delta_{1} \sum_{i=b}^{a} \sum_{j=b}^{c-1} w e_{a}^{i} h_{i}^{i+j-b+1} h_{i+j-b+1}^{j} h_{j}^{c} \\
& =w \sum_{j=b}^{c-1}\left(\sum_{i=b}^{a-1} e_{a}^{i+1} e_{i+1}^{i+j-b+1} h_{i+j-b+1}^{j}-\sum_{i=b+1}^{a} e_{a}^{i} e_{i}^{i+j-b} h_{i+j-b}^{j}\right) h_{j}^{c} \\
& +w \sum_{i=b}^{a} e_{a}^{i}\left(-\sum_{j=b+1}^{c-1} h_{i}^{i+j-b} e_{i+j-b}^{j} h_{j}^{c}+\sum_{j=b}^{c-2} h_{i}^{i+j-b+1} e_{i+j-b+1}^{j+1} h_{j+1}^{c}\right)
\end{aligned}
$$




$$
\begin{aligned}
& +w \sum_{i=b+1}^{a} \sum_{j=b}^{c-1} e_{a}^{i} h_{i}^{i+j-b+1} h_{i+j-b+1}^{j+1} e_{j+1}^{c} \\
= & 0+0+w \sum_{i=b+1}^{a} \sum_{j=b+1}^{c} e_{a}^{i} h_{i}^{i+j-b} h_{i+j-b}^{j} e_{j}^{c} \\
= & S\left(w e_{a}^{b+1} e_{b+1}^{c}\right)=S \delta_{1}\left(w e_{a}^{b} h_{b}^{c}\right) .
\end{aligned}
$$

This completes the proof.

We now come to the key argument which explains how the homologies for different $n$ are related.

Proposition 7.5 For all $n \geq 1$, the chain map $S$ induces an isomorphism

$$
H X_{*}^{(j)}(n) \stackrel{\simeq}{\longrightarrow} H X_{*}^{(j-2)}(n+1) .
$$

Proof If $\alpha$ is a generator of $C X_{*}(n+1)$ corresponding to the corner sequence $a_{0}, a_{1}, \ldots, a_{2 n+2}=a_{0}$, define the "degree"

$$
\operatorname{deg}(\alpha):=\sum_{i=0}^{2 n-2}\left|a_{i}-a_{i+1}\right|+\left|a_{2 n+1}-a_{2 n+2}\right| .
$$

In other words the degree is the sum of the lengths of the edges, except for the two edges that could arise from splicing a generator of $C X_{*}(n)$. This "degree" defines an increasing filtration on $C X_{*}(n+1)$ and hence gives rise to a spectral sequence $E_{*, *}^{*}$. By equation (49), the filtration is bounded in terms of the index by

$$
0 \leq \operatorname{deg}(\alpha) \leq I(\alpha)+2 n .
$$

It follows that the spectral sequence $E_{*, *}^{*}$ converges to $H X_{*}(n+1)$.

For $i, j \in \mathbb{Z}$, let $C_{*}(i, j)$ denote the direct limit of $C_{*}(\Lambda)$, where $\Lambda$ is an open admissible path on the $x$-axis parametrized by the interval $(\pi / 2+2 \pi(n-1), \pi / 2+$ $2 \pi n)$ and with endpoints $(i, 0)$ and $(j, 0)$. That is, generators of $C_{*}(i, j)$ have underlying admissible paths that start at $(i, 0)$, go in the negative $x$ direction by some (possibly zero) amount, then turn by $\pi$ and go in the positive $x$ direction by some (possibly zero) amount to $(j, 0)$. In this notation, the $E^{1}$ term of the spectral sequence is given by

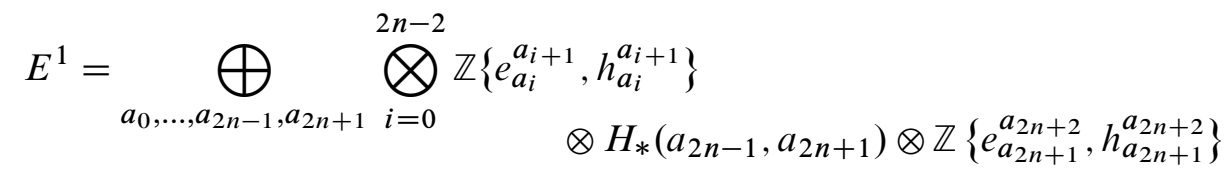


where $a_{i} \geq a_{i+1}$ for $i \neq 2 n$ even, $a_{i} \leq a_{i+1}$ for $i \neq 2 n-1$ odd, and $a_{2 n+2}=a_{0}$. By Propositions 5.6 and 5.7,

$$
H_{*}(i, j)=\left\{\begin{array}{cc}
0, & i \neq j, \\
\mathbb{Z}\left\{h_{i}^{i-1} h_{i-1}^{i}\right\}, & i=j .
\end{array}\right.
$$

By equations (48), (50) and (51), there is an isomorphism of $\mathbb{Z}$-modules

$$
C X_{*}(n) \simeq E^{1} .
$$

Now we can filter $C X_{*}(n)$ by the sum of the lengths of all the edges. This filtration gives rise to a spectral sequence ' $E_{*, *}^{*}$ converging (at the third term) to $H X_{*}(n)$ with

$$
{ }^{\prime} E^{1}=C X_{*}(n) .
$$

The splicing chain map $S: C X_{*}(n) \rightarrow C X_{*}(n+1)$ respects the above filtrations and therefore induces a morphism of spectral sequences ' $E_{*, *}^{*} \rightarrow E_{*, *}^{*}$. On the first term, $S$ induces the isomorphism ' $E^{1} \stackrel{\simeq}{\longrightarrow} E^{1}$ given by (52) and (53), because by the definition of $S$, the only term in which no old edge is shortened is the term in which two ' $h$ ', edges of length one are spliced in. Therefore $S$ induces an isomorphism ${ }^{\prime} E^{\infty} \stackrel{\simeq}{\longrightarrow} E^{\infty}$, hence an isomorphism $H X_{*}(n) \stackrel{\simeq}{\longrightarrow} H X_{*}(n+1)$.

Corollary 7.6 For $(i, j) \neq(0,-2 n)$,

$$
H X_{i}^{(j)}(n) \simeq\left\{\begin{array}{l}
\mathbb{Z}, i=2 k, 2 k+1 ; j=2 k-2 n+2 ; k \in \mathbb{Z}_{\geq 0}, \\
0, \text { otherwise. }
\end{array}\right.
$$

Proof Applying Proposition 5.9 to $x$-axis polygons and taking the direct limit proves the claim for $n=1$. It follows by Proposition 7.5 that the claim holds for all $n$.

We will also need the following lemma in Section 7.3:

Lemma 7.7 For appropriate choices of the angles used to define $U$,

$$
S U=U S: C X_{i}^{(j)}(n) \longrightarrow C X_{i-2}^{(j-4)}(n+1) .
$$

Proof Define $U$ on $C_{*}(2 \pi n ; 0)$ using $\theta=\pi / 2+(2 n-2) \pi$, and define $U$ on $C_{*}(2 \pi(n+1) ; 0)$ using $\theta=\pi / 2+(2 n-1) \pi$. We will check that equation (54) holds when applied to a generator of the form $w e_{a}^{b} h_{b}^{c}$; the other cases are very similar. Note 
that $U$ acts on a generator of $C X_{*}(n)$ simply by shrinking the two edges adjacent to $c_{\theta}$ by one, preserving the edge labels. Thus

$$
\begin{aligned}
U S\left(w e_{a}^{b} h_{b}^{c}\right) & =U\left(\sum_{i=b}^{a} \sum_{j=b}^{c-1} w e_{a}^{i} h_{i}^{i+j-b+1} h_{i+j-b+1}^{j} h_{j}^{c}\right) \\
& =\sum_{i=b+1}^{a} \sum_{j=b+1}^{c-1} w e_{a}^{i} h_{i}^{i+j-b} h_{i+j-b}^{j} h_{j}^{c} \\
& =S\left(w e_{a}^{b+1} h_{b+1}^{c}\right) \\
& =S U\left(w e_{a}^{b} h_{b}^{c}\right),
\end{aligned}
$$

which completes the proof.

\subsection{Inclusion}

The proof of Theorem 7.1 will proceed by taking a direct limit, for which purpose we will need the following technical lemma about how $H_{*}(\Lambda)$ behaves under inclusion of polygonal regions.

Lemma 7.8 Let $\Lambda$ and $\Lambda^{\prime}$ be $n$-convex paths with $\Lambda^{\prime} \leq \Lambda$. If $(i, j) \neq(0,-2 n)$, and if $k_{\Lambda^{\prime}}$ is sufficiently large with respect to $i$ and $j$, then inclusion induces an isomorphism

$$
H_{i}^{(j)}\left(\Lambda^{\prime}\right) \stackrel{\simeq}{\longrightarrow} H_{i}^{(j)}(\Lambda)
$$

To prove Lemma 7.8, we will need to compute $H_{*}(\Lambda)$ for certain special open admissible paths $\Lambda$ on the $x$-axis. Similarly to the notation in Section 7.1, we can write

$$
\Lambda=\lambda_{a_{0}}^{a_{1}} \cdots \lambda_{a_{k-1}}^{a_{k}}
$$

where $\lambda_{a_{i}}^{a_{i+1}}$ denotes an edge from $\left(a_{i}, 0\right)$ to $\left(a_{i+1}, 0\right)$ occuring at angle $\left(i+i_{0}\right) \pi$ for some integer $i_{0}$. Here we are not requiring that $a_{0}=a_{k}$.

Definition 7.9 A spiral is a nonconstant open admissible path on the $x$-axis, $\Lambda=$ $\lambda_{a_{0}}^{a_{1}} \cdots \lambda_{a_{k-1}}^{a_{k}}$, such that $k \geq 1$, and if $k>1$ then

$$
\begin{aligned}
\left|a_{0}-a_{1}\right| & >\left|a_{1}-a_{2}\right|, \\
\left|a_{i}-a_{i+1}\right| & \geq\left|a_{i+1}-a_{i+2}\right|, \quad i=1, \ldots, k-2 .
\end{aligned}
$$

Lemma 7.10 If $\Lambda$ is a spiral, then $H_{*}(\Lambda)=\mathbb{Z}\left\{E_{\Lambda}, H_{\Lambda}\right\}$. 
Proof If $\Lambda$ has only edge then the lemma is immediate. Otherwise let $c$ be the corner of $\Lambda$ preceding the last edge. Then $c$ splits $\Lambda=\Lambda_{1} \Lambda_{2}$ where $\Lambda_{1}, \Lambda_{2}$, and $\Lambda \backslash c$ are all spirals. The lemma follows by induction using the long exact sequence (27), as in the proof of Proposition 5.6.

Definition 7.11 A semi-spiral is an open admissible path on the $x$-axis, $\Lambda=$ $\lambda_{a_{0}}^{a_{1}} \cdots \lambda_{a_{k-1}}^{a_{k}} \lambda_{a_{k}}^{a_{k+1}}$, with $k \geq 2$, satisfying the conditions (55) and

$$
\left|a_{0}-a_{1}\right| \leq\left|a_{k}-a_{k+1}\right| \text {. }
$$

If $\Lambda$ is a semi-spiral, define $V_{\Lambda}, W_{\Lambda} \in C_{*}(\Lambda)$ by

$$
\begin{aligned}
V_{\Lambda} & :=E_{\lambda_{a_{0} \ldots}^{a_{1} \ldots} \lambda_{a_{k-2}}^{a_{k-1}}} e_{a_{k-1}}^{a_{k-1}} h_{a_{k-1}}^{a_{k+1}}, \\
W_{\Lambda} & :=H_{\lambda_{a_{0}}^{a_{1} \ldots \lambda_{a_{k-2}}} a_{k-1}}^{a_{a_{k-1}}} h_{a_{k-1}}^{a_{k+1}} .
\end{aligned}
$$

Note that the generator $V_{\Lambda}$, and the generators in the sum $W_{\Lambda}$, each have one kink.

Lemma 7.12 If $\Lambda$ is a semi-spiral, then $H_{*}(\Lambda)=\mathbb{Z}\left\{E_{\Lambda}, H_{\Lambda}, V_{\Lambda}, W_{\Lambda}\right\}$.

Proof We use induction on the length of $\Lambda$. If $\Lambda$ has only two edges, so that $a_{1}=a_{2}=\cdots=a_{k}$, then the lemma follows by inspection. Namely, $C_{*}(\Lambda)$ has only four generators $e e, e h, h e, h h$ which correspond to $E_{\Lambda}, V_{\Lambda}, H_{\Lambda}-V_{\Lambda}$, and $W_{\Lambda}$ above. The differential vanishes here because the corner between the two edges is a kink since $k \geq 2$.

If $\Lambda$ has more than two edges, let $c$ denote the corner preceding the second-to-last edge. Then $c$ splits $\Lambda=\Lambda_{1} \Lambda_{2}$ where $\Lambda_{2}$ and $\Lambda \backslash c$ are semi-spirals, while $\Lambda_{1}$ is a spiral. We now use the long exact sequence (27). The connecting homomorphism

$$
\partial_{c}: H_{*}\left(C_{*}\left(\Lambda_{1}\right) \otimes C_{*}\left(\Lambda_{2}\right)\right) \longrightarrow H_{*}(\Lambda \backslash c)
$$

is given by the equations (30) together with

$$
\begin{aligned}
& E_{\Lambda_{1}} \otimes V_{\Lambda_{2}} \longmapsto 0, \\
& H_{\Lambda_{1}} \otimes V_{\Lambda_{2}},-E_{\Lambda_{1}} \otimes W_{\Lambda_{2}} \longmapsto V_{\Lambda \backslash c}, \\
& H_{\Lambda_{1}} \otimes W_{\Lambda_{2}} \longmapsto-W_{\Lambda \backslash c}
\end{aligned}
$$

So $H_{*}(\Lambda)$ is freely generated by $E_{\Lambda_{1}} E_{\Lambda_{2}}=E_{\Lambda}, E_{\Lambda_{1}} H_{\Lambda_{2}}+H_{\Lambda_{1}} E_{\Lambda_{2}}=H_{\Lambda}$, $E_{\Lambda_{1}} V_{\Lambda_{2}}=V_{\Lambda}$, and $H_{\Lambda_{1}} V_{\Lambda_{2}}+E_{\Lambda_{1}} W_{\Lambda_{2}}=W_{\Lambda}$. 
Proof of Lemma 7.8 By Proposition 2.13, $P_{\Lambda^{\prime}}$ is obtained from $P_{\Lambda}$ by a finite sequence of corner roundings. We can assume that $P_{\Lambda^{\prime}}$ is obtained from $P_{\Lambda}$ by rounding a single corner $c$ (the general case then follows by induction). Choose $\theta \in \mathbb{R} / 2 \pi \mathbb{Z}$ such that $c$ is the distinguished corner $c_{\theta}$ of $P_{\Lambda}$. By Proposition 6.14, we can assume that $\Lambda=\Lambda_{0}$ and $\Lambda^{\prime}=\Lambda_{0}^{\prime}$ are on the $x$-axis. Without loss of generality, $P_{\Lambda_{0}}=[1, k] \times\{0\}$ and $P_{\Lambda_{0}^{\prime}}=[1, k-1] \times\{0\}$.

Let $c_{1}, \ldots, c_{n}$ denote the corners of $\Lambda_{0}$ at $(k, 0)$, in counterclockwise order. For $p, q \in\{1, \ldots, n\}$ distinct, let $\Lambda_{p, q}$ denote the open path given by the portion of $\Lambda_{0}$ starting at $c_{p}$ and ending at $c_{q}$, with the intermediate corners $c_{p+1}, \ldots, c_{q-1}$ rounded. There is then a decomposition of $\mathbb{Z}$-modules

$$
\begin{aligned}
& C_{*}\left(\Lambda_{0}\right)= \\
& \quad C_{*}\left(\Lambda_{0}^{\prime}\right) \oplus \bigoplus_{m=1}^{n} \bigoplus_{1 \leq p_{1}<\cdots<p_{m} \leq n} C_{*}\left(\Lambda_{p_{1}, p_{2}}\right) \otimes \cdots \otimes C_{*}\left(\Lambda_{p_{m-1}, p_{m}}\right) \otimes C_{*}\left(\Lambda_{p_{m}, p_{1}}\right) .
\end{aligned}
$$

Furthermore, $m$ defines an increasing filtration on $C_{*}\left(\Lambda_{0}\right)$, where we interpret the $C_{*}\left(\Lambda_{0}^{\prime}\right)$ summand as corresponding to $m=0$. (One can regard $m$ as a weighted count of the times that an admissible path $\lambda \leq \Lambda_{0}$ stops at the point $(k, 0)$.) Thus we obtain a spectral sequence $E_{*, *}^{*}$ converging to $H_{*}\left(\Lambda_{0}\right)$. The $E^{1}$ term is given by

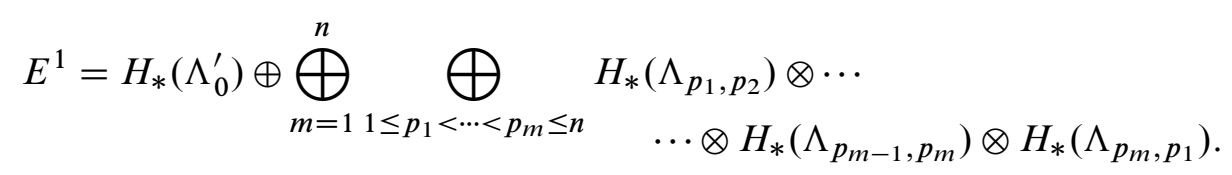

Here we have used the fact that $H_{*}\left(\Lambda_{p, q}\right)$ has no torsion, which is justified below.

The key now is to compute the indices of the generators of $H_{*}\left(\Lambda_{p, q}\right)$. Let $n^{\prime}=q-p$ if $p<q$, and let $n^{\prime}=p+n-q$ if $p \geq q$. If $n^{\prime}=1$, then $H_{*}\left(\Lambda_{p, q}\right)$ is given by Proposition 5.7. The indices of the generators are

$$
I\left(Z_{1}((k, 0),(k-1,0))\right)=0, \quad I\left(E_{\Lambda_{p, q}}\right)=2 k-2, \quad I\left(H_{\Lambda_{p, q}}\right)=2 k-3 .
$$

If $n^{\prime}>1$, then $\Lambda_{p, q}$ is a semi-spiral, so $H_{*}\left(\Lambda_{p, q}\right)$ is given by Lemma 7.12. (Note that $k$ here is different from the $k$ in the definition of semi-spiral.) The indices of the generators of $H_{*}\left(\Lambda_{p, q}\right)$ are

$$
\begin{aligned}
I\left(E_{\Lambda_{p, q}}\right) & =2 n^{\prime}(k-2)+2, \\
I\left(H_{\Lambda_{p, q}}\right) & =2 n^{\prime}(k-2)+1, \\
I\left(V_{\Lambda_{p, q}}\right) & =2\left(n^{\prime}-1\right)(k-2)+1, \\
I\left(W_{\Lambda_{p, q}}\right) & =2\left(n^{\prime}-1\right)(k-2) .
\end{aligned}
$$


In particular, the index of each generator, except for $Z_{1}((k, 0),(k-1,0))$, is a linearly increasing function of $k$. Therefore the index of every $m>0$ homology class in the $E^{1}$ term (56) is a linearly increasing function of $k$, except for the product of $n Z_{1}$ 's in the $m=n$ piece, which lives in $C_{0}^{(-2 n)}\left(\Lambda_{0}\right)$. It follows that if $k$ is sufficiently large with respect to $(i, j) \neq(0,-2 n)$, then the map

$$
H_{i}^{(j)}\left(\Lambda_{0}^{\prime}\right) \longrightarrow H_{i}^{(j)}\left(\Lambda_{0}\right)
$$

induced by the inclusion is an isomorphism.

\subsection{The direct limit}

We can now complete the calculation of $\widetilde{H}_{*}(2 \pi n ; 0)$.

Lemma 7.13 If $(i, j) \neq(0,-2 n)$, then the inclusion $C X_{*}^{(j)}(n) \rightarrow C_{*}^{(j)}(2 \pi n ; 0)$ induces an isomorphism

$$
H X_{i}^{(j)}(n) \stackrel{\simeq}{\longrightarrow} H_{i}^{(j)}(2 \pi n ; 0)
$$

Proof Let $\Lambda_{0}$ be an $n$-convex path on the $x$-axis, and consider the commutative diagram

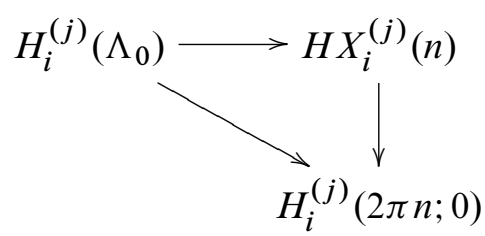

where the arrows are induced by the inclusions of chain complexes. The homology $H_{i}^{(j)}(2 \pi n ; 0)$ is given by the direct limit

$$
H_{i}^{(j)}(2 \pi n ; 0)=\lim _{\longrightarrow} H_{i}^{(j)}(\Lambda)
$$

over $n$-convex paths $\Lambda$. It follows by Lemma 7.8 that if $k_{\Lambda_{0}}$ is sufficiently large with respect to $(i, j)$, then the diagonal arrow in the diagram (57) is an isomorphism, and likewise the horizontal arrow is an isomorphism. Hence the vertical arrow is an isomorphism.

Proof of Theorem 7.1 (a) To start, we obtain an isomorphism of $\mathbb{Z}$-modules

$$
H_{0}^{(-2 n)}(2 \pi n ; 0) \simeq \mathcal{I}\left(\mathbb{Z}^{2}\right)
$$


by taking the direct limit (58) and applying Proposition 6.19. This is in fact an isomorphism of $\mathbb{Z}\left[\mathbb{Z}^{2}\right]$-modules, because by the definition of $Z_{n}(a, b)$, translation by $w \in \mathbb{Z}^{2}$ sends $Z_{n}(a, b)$ to $Z_{n}(a+w, b+w)$.

For the rest of the proof suppose that $(i, j) \neq(0,-2 n)$. By Corollary 7.6 and Lemma 7.13 , we have

$$
H_{i}^{(j)}(2 \pi n ; 0) \simeq \begin{cases}\mathbb{Z}, & i=2 k, 2 k+1 \geq 0, j=2 k-2 n+2, \\ 0, & \text { otherwise }\end{cases}
$$

To complete the proof of part (a), we must show that translations act by the identity on $H_{i}^{(j)}(2 \pi n ; 0)$. Taking the direct limit of Proposition 5.9 applied to $x$-axis polygons shows that $H X_{i}^{(j)}(1)$, if nonzero, is generated by $E_{\Lambda_{0}}$ or $H_{\Lambda_{0}}$ where $\Lambda_{0}$ is on the $x$-axis. By Lemma 5.8, the homology class of $E_{\Lambda_{0}}$ or $H_{\Lambda_{0}}$ in $H X_{i}^{(j)}(1)$ depends only on the number of lattice points enclosed by $\Lambda_{0}$. Therefore translation in the $x$ direction acts by the identity on $H X_{i}^{(j)}(1)$. By Definition 7.3, the splicing isomorphism $S$ commutes with translation in the $x$ direction. By Proposition 7.5, it follows that translation in the $x$ direction acts by the identity on $H X_{i}^{(j)}(n)$ for all $n$. So by Lemma 7.13 , translation in the $x$ direction acts by the identity on $H_{i}^{(j)}(2 \pi n ; 0)$. By the symmetry of Lemma 6.20, all translations act by the identity on $H_{i}^{(j)}(2 \pi n ; 0)$.

(b) By Lemma 7.13 and part (a), it is enough to show that if $i \geq 2$ and $j \neq 2-2 n$ then $U$ induces an isomorphism

$$
U: H X_{i}^{(j)}(n) \stackrel{\simeq}{\longrightarrow} H X_{i-2}^{(j-2)}(n) .
$$

When $n=1$, we know that (59) is an isomorphism by the above description of the generators of $H X_{i}^{(j)}(1)$ and Proposition 4.2(c). It then follows from Proposition 7.5 and Lemma 7.7 that (59) is an isomorphism for all $n$.

\section{Calculation of $\bar{H}_{*}(2 \pi n ; 0)$}

This section is devoted to computing the homology of the complex $\bar{C}_{*}(2 \pi n ; 0)$ over $\mathbb{Z}$, defined in (17), in which we mod out by translation of polygons. This will prove parts (b) and (c) of Theorem 1.3.

\subsection{The universal coefficient spectral sequence}

The homology $\bar{H}_{*}(2 \pi n ; 0)$ is partially but not entirely determined by $\widetilde{H}_{*}(2 \pi n ; 0)$. The precise relation between $\widetilde{H}_{*}$ and $\bar{H}_{*}$ is expressed by the "universal coefficient spectral sequence", whose construction we now recall. 
In general let $R$ be a commutative ring, let $\left(C_{*}, \delta\right)$ be a chain complex of projective $R$-modules, and let $A$ be an $R$-module. The task at hand is to relate the homology of $\left(C_{*} \otimes_{R} A, \delta \otimes 1\right)$ to the homology of $C_{*}$. Let

$$
\stackrel{\partial}{\longrightarrow} P_{2} \stackrel{\partial}{\longrightarrow} P_{1} \stackrel{\partial}{\longrightarrow} P_{0} \longrightarrow A \longrightarrow 0
$$

be a projective resolution of $A$ in the category of $R$-modules. Recall that if $M$ is another $R$-module then $\operatorname{Tor}_{*}(M, A)$ is defined to be the homology of the complex $\left(M \otimes_{R} P_{*}, 1 \otimes \partial\right)$. This satisfies $\operatorname{Tor}_{0}(M, A)=M \otimes_{R} A$, and if $M$ is projective then $\operatorname{Tor}_{i}(M, A)=0$ for $i>0$. Now form the double complex

$$
C_{i, j}:=C_{j} \otimes_{R} P_{i}
$$

This has horizontal, vertical, and total differentials

$$
d_{h}:=1 \otimes \partial, \quad d_{v}:=\delta \otimes 1, \quad d:=d_{h}+(-1)^{i} d_{v} .
$$

Filtering the double complex by $j$ gives a spectral sequence with

$$
E_{i, j}^{1}=\operatorname{Tor}_{i}\left(C_{j}, A\right)=\left\{\begin{array}{cc}
C_{j} \otimes_{R} A, & i=0 \\
0, & i>0
\end{array}\right.
$$

so the homology of the double complex is $H_{*}\left(C_{*} \otimes_{R} A\right)$. The universal coefficient spectral sequence is obtained by filtering the double complex by $i$ instead. This spectral sequence satisfies

$$
\begin{aligned}
& E_{i, j}^{1}=H_{j}\left(C_{*}\right) \otimes_{R} P_{i}, \\
& E_{i, j}^{2}=\operatorname{Tor}_{i}\left(H_{j}\left(C_{*}\right), A\right),
\end{aligned}
$$

and by the previous calculation converges to $H_{*}\left(C_{*} \otimes_{R} A\right)$.

We now specialize to the case $R=\mathbb{Z}\left[\mathbb{Z}^{2}\right], C_{*}=\widetilde{C}_{*}(2 \pi n ; 0)$, and $A=\underline{\mathbb{Z}}$. In the rest of this section, the tensor product is understood to be over $\mathbb{Z}\left[\mathbb{Z}^{2}\right]$.

We begin by computing the relevant Tor's, ie the $E^{2}$ term of the universal coefficient spectral sequence. We fix the following projective resolution of $\mathbb{Z}$ :

$$
\begin{gathered}
0 \longrightarrow \mathbb{Z}\left[\mathbb{Z}^{2}\right]\{\gamma\} \stackrel{\partial}{\longrightarrow} \mathbb{Z}\left[\mathbb{Z}^{2}\right]\{\alpha, \beta\} \stackrel{\partial}{\longrightarrow} \mathbb{Z}\left[\mathbb{Z}^{2}\right]\{\tau\} \longrightarrow \mathbb{Z} \longrightarrow 0, \\
\partial \gamma:=(y-1) \alpha-(x-1) \beta, \quad \partial \alpha:=(x-1) \tau, \quad \partial \beta:=(y-1) \tau .
\end{gathered}
$$


After tensoring with $\underline{\mathbb{Z}}$, the differential becomes 0 , so

$$
\operatorname{Tor}_{i}(\underline{\mathbb{Z}}, \mathbb{Z}) \simeq\left\{\begin{array}{cl}
\mathbb{Z}, & i=0, \\
\mathbb{Z} \oplus \mathbb{Z}, & i=1, \\
\mathbb{Z}, & i=2, \\
0, & i>2 .
\end{array}\right.
$$

We compute $\operatorname{Tor}_{*}\left(\mathcal{I}\left(\mathbb{Z}^{2}\right), \underline{\mathbb{Z}}\right)$ by the symmetry $\operatorname{Tor}_{*}\left(\mathcal{I}\left(\mathbb{Z}^{2}\right), \underline{\mathbb{Z}}\right)=\operatorname{Tor}_{*}\left(\underline{\mathbb{Z}}, \mathcal{I}\left(\mathbb{Z}^{2}\right)\right)$. The $\mathbb{Z}\left[\mathbb{Z}^{2}\right]$-module $\mathcal{I}\left(\mathbb{Z}^{2}\right)$ has a presentation with two generators $a=x-1$ and $b=y-1$ and the single relation $(y-1) a-(x-1) b=0$. Therefore

$$
\operatorname{Tor}_{i}\left(\mathcal{I}\left(\mathbb{Z}^{2}\right), \underline{\mathbb{Z}}\right)=\operatorname{Tor}_{i}\left(\underline{\mathbb{Z}}, \mathcal{I}\left(\mathbb{Z}^{2}\right)\right) \simeq\left\{\begin{array}{cc}
\underline{\mathbb{Z}} \underline{\mathbb{Z}}, & i=0, \\
\mathbb{Z}, & i=1, \\
0, & i>0
\end{array}\right.
$$

In terms of the projective resolution of $\mathbb{Z}$, it turns out that $\operatorname{Tor}_{0}\left(\mathcal{I}\left(\mathbb{Z}^{2}\right), \mathbb{Z}\right)$ is generated by $(x-1) \otimes \tau$ and $(y-1) \otimes \tau$, while $\operatorname{Tor}_{1}\left(\mathcal{I}\left(\mathbb{Z}^{2}\right), \underline{Z}\right)$ is generated by $(y-1) \otimes \alpha-$ $(x-1) \otimes \beta$.

Now the only possibly nonzero higher differential in the universal coefficient spectral sequence is, for $j \geq 0$,

$$
\begin{gathered}
d_{2}: E_{2, j}^{2} \longrightarrow E_{0, j+1}^{2}, \\
\underline{\mathbb{Z}} \simeq \operatorname{Tor}_{2}\left(\widetilde{H}_{j}(2 \pi n ; 0), \underline{\mathbb{Z}}\right) \longrightarrow \operatorname{Tor}_{0}\left(\widetilde{H}_{j+1}(2 \pi n ; 0), \underline{\mathbb{Z}}\right) \simeq \mathbb{Z} .
\end{gathered}
$$

To compute this differential, the only explicit calculation we will need is given by the following lemma, which will be proved in Section 8.3.

Lemma 8.1 If $p$ is a cycle generating $\widetilde{H}_{0}^{(2-2 n)}(2 \pi n ; 0) \simeq \mathbb{Z}$, and if $s$ and $t$ are chains with $\delta s=(x-1) p$ and $\delta t=(y-1) p$, then $(y-1) s-(x-1) t$ generates $\widetilde{H}_{1}^{(2-2 n)}(2 \pi n ; 0) \simeq \mathbb{Z}$.

Lemma 8.2 The differential (62) is zero if $j$ is odd, and an isomorphism if $j \geq 0$ is even.

Proof We first derive a general formula for $d_{2}$. Let $p \in \widetilde{C}_{j}(2 \pi n ; 0)$ be a cycle generating the $\mathbb{Z}$ component of $\widetilde{H}_{j}(2 \pi n ; 0)$. Referring back to the generator $\gamma$ of our projective resolution of $\mathbb{Z}$, we want to compute $d_{2}[p \otimes \gamma]$. This is done by "zig-zagging". First we calculate the horizontal differential

$$
d_{h}(p \otimes \gamma)=p \otimes((y-1) \alpha-(x-1) \beta) \in C_{1, j}
$$


Next we need to find an element

$$
-t \otimes \alpha+s \otimes \beta \in C_{1, j+1}
$$

with $-d_{v}(-t \otimes \alpha+s \otimes \beta)=d_{h}(p \otimes \gamma)$. That is, we need to find $s, t \in \widetilde{C}_{j+1}(2 \pi n ; 0)$ with

$$
\delta s=(x-1) p, \quad \delta t=(y-1) p
$$

in $\widetilde{C}_{j}(2 \pi n ; 0)$. For $s$ and $t$ satisfying (63), we then have

$$
\begin{aligned}
d_{2}[p \otimes \gamma] & =\left[d_{h}(-t \otimes \alpha+s \otimes \beta)\right] \\
& =[(y-1) s-(x-1) t) \otimes \tau] .
\end{aligned}
$$

If $j=0$, then Lemma 8.1 and equation (64) imply that $d_{2}[p \otimes \gamma]$ is a generator of $\operatorname{Tor}_{0}\left(\widetilde{H}_{1}(2 \pi n ; 0), \mathbb{Z}\right) \simeq \underline{\mathbb{Z}}$.

Suppose next that $j=2 k$. Since the chain map $U$ commutes with $x$ and $y$ according to Proposition 4.2(b), it follows by functoriality or by equation (64) that the induced map on homology $U_{*}$ commutes with the differential $d_{2}$. By Theorem 7.1(b), we are done in this case by induction on $k$.

Finally, if $j=2 k+1$, we show that the differential (62) is zero by considering the decomposition into subcomplexes from Definition 5.1. First, Theorem 7.1(a) implies that

$$
\widetilde{H}_{j}(2 \pi n ; 0)=\widetilde{H}_{j}^{(2 k-2 n+2)}(2 \pi n ; 0),
$$

so we can take $p \in \widetilde{C}_{j}^{(2 k-2 n+2)}(2 \pi n ; 0)$ and $s, t \in \widetilde{C}_{j+1}^{(2 k-2 n+2)}(2 \pi n ; 0)$. But Theorem 7.1(a) also implies that

$$
\widetilde{H}_{j+1}(2 \pi n ; 0)=\widetilde{H}_{j+1}^{(2 k-2 n+4)}(2 \pi n ; 0) .
$$

Hence the cycle $(y-1) s-(x-1) t$ is nullhomologous in $\widetilde{C}_{*}(2 \pi n ; 0)$, so by equation (64), $d_{2}[p \otimes \gamma]=0$.

Proof of Theorem 1.3(b) By equations (60) and (61) and Lemma 8.2, the $E^{2}$ term of the universal coefficient spectral sequence looks like this:

\begin{tabular}{|c|c|c|}
$\vdots$ & \multicolumn{1}{c}{$\vdots$} & $\vdots$ \\
\hline$\underline{\mathbb{Z}}$ & $\underline{\mathbb{Z}}^{2}$ & $\underline{\mathbb{Z}}$ \\
\hline$\underline{\mathbb{Z}}$ & $\underline{\mathbb{Z}}^{2}$ & $\underline{\mathbb{Z}}$ \\
\hline$\underline{\mathbb{Z}}$ & $\underline{\mathbb{Z}}^{2}$ & $\underline{\mathbb{Z}}$ \\
\hline$\underline{\mathbb{Z}}^{3}$ & $\underline{\mathbb{Z}}^{3}$ & $\underline{\mathbb{Z}}$ \\
\hline
\end{tabular}


Here the $d_{2}$ arrows drawn are isomorphisms, while the others are zero. It follows immediately that the spectral sequence converges to $\underline{\mathbb{Z}}^{3}$ in each nonnegative degree. This is $\bar{H}_{*}(2 \pi n ; 0)$ as a $\mathbb{Z}\left[\mathbb{Z}^{2}\right]$-module; as a $\mathbb{Z}$-module, it is simply $\mathbb{Z}^{3}$ in each nonnegative degree.

More explicitly, the proof of Theorem 1.3(b) shows the following.

Proposition 8.3 For $k \geq 0$, let $p_{k}$ be a cycle generating $\widetilde{H}_{2 k}^{(2 k-2 n+2)}(2 \pi n ; 0)$, and let $q_{k}$ be a cycle generating $\widetilde{H}_{2 k+1}^{(2 k-2 n+2)}(2 \pi n ; 0)$. Then $\bar{H}_{*}(2 \pi n ; 0)$ is freely generated over $\mathbb{Z}$ by the images in $\bar{C}_{*}(2 \pi n ; 0)$ of the following chains in $\widetilde{C}_{*}(2 \pi n ; 0)$ :

- The index $2 k$ cycle $p_{k}$, for each $k \geq 0$.

- Two index $2 k+1$ chains $s_{k}$ and $t_{k}$ with $\delta s_{k}=(x-1) p_{k}$ and $\delta t_{k}=(y-1) p_{k}$, for each $k \geq 0$.

- The index 0 cycles $u_{0}:=Z_{n}((1,0),(0,0))$ and $v_{0}:=Z_{n}((0,1),(0,0))$.

- Two index $2 k$ chains $u_{k}$ and $v_{k}$ with $\delta u_{k}=(x-1) q_{k-1}$ and $\delta v_{k}=(y-1) q_{k-1}$, for each $k \geq 1$.

- An index $2 k+1$ chain $w_{k}$ with $\delta w_{k}=(y-1) u_{k}-(x-1) v_{k}$ for each $k \geq 0$.

Moreover, the corresponding homology classes in $\bar{H}_{*}(2 \pi ; 0)$ do not depend on any of the choices of $p_{k}, q_{k}, s_{k}, t_{k}, u_{k}, v_{k}, w_{k}$.

Note for example that the cycle $q_{k}$ maps to zero in $\bar{H}_{*}(2 \pi n ; 0)$, because we saw above that $\pm q_{k}$ is homologous in $\widetilde{C}_{*}(2 \pi n ; 0)$ to $(y-1) s_{k}-(x-1) t_{k}$, and $(y-1)$ and $(x-1)$ are in the kernel of the augmentation map $\mathbb{Z}\left[\mathbb{Z}^{2}\right] \rightarrow \mathbb{Z}$.

\subsection{The action of $U$ on $\bar{H}_{*}(2 \pi n ; 0)$}

We now prove Theorem 1.3(c). Fix $\theta \in \mathbb{R} / 2 \pi n \mathbb{Z}$ with $\tan \theta$ irrational in the definition of $U$. We will use the following lemma which is proved in Section 8.3.

Lemma 8.4 There exist $q_{0} \in \widetilde{C}_{1}^{(2-2 n)}(2 \pi n ; 0)$ generating $\widetilde{H}_{1}^{(2-2 n)}(2 \pi n ; 0)$ and $u_{1}, v_{1} \in \widetilde{C}_{2}^{(2-2 n)}(2 \pi n ; 0)$ such that

$$
\begin{array}{cl}
\delta u_{1}=(x-1) q_{0}, & \delta v_{1}=(y-1) q_{0} \\
U u_{1}=Z_{n}((1,0),(0,0)), & U v_{1}=Z_{n}((0,1),(0,0)) .
\end{array}
$$


Proof of Theorem 1.3(c) It is enough to show that for any $k \geq 0$, one can make the choices in Proposition 8.3 for $k$ and $k+1$ such that

$$
\begin{gathered}
U p_{k+1}=p_{k}, \quad U s_{k+1}=s_{k}, \quad U t_{k+1}=t_{k}, \\
U u_{k+1}=u_{k}, \quad U v_{k+1}=v_{k}, \quad U w_{k+1}=w_{k}
\end{gathered}
$$

in $\widetilde{C}_{*}(2 \pi n ; 0)$.

First choose any $p_{k+1}, q_{k+1}, s_{k+1}, t_{k+1}, u_{k+1}, v_{k+1}, w_{k+1}$. By Theorem 7.1(b), we can choose $p_{k}:=U p_{k+1}$ and $q_{k}:=U q_{k+1}$. Since $U$ is a translation-invariant chain map, we can then choose $s_{k}:=U s_{k+1}$ and $t_{k}:=U t_{k+1}$, and if $k>0$ we can also choose $u_{k}:=U u_{k+1}, v_{k}:=U v_{k+1}$, and $w_{k}:=U w_{k+1}$.

To complete the proof, it is enough to show that for suitable choices,

$$
U u_{1}=u_{0}, \quad U v_{1}=v_{0}, \quad U w_{1}=w_{0} .
$$

We can obtain the first two conditions in (69) by Lemma 8.4. Then to obtain the third condition, given any choice of $w_{1}$, we can choose $w_{0}:=U w_{1}$.

\subsection{Some explicit homology generators and relations}

This subsection is devoted to the proofs of Lemmas 8.1 and 8.4 above.

Definition 8.5 Let $a, b \in \mathbb{Z}^{2}$ be lattice points with $a-b$ indivisible. Choose $\theta \in \mathbb{R}$ such that the line from $a$ to $b$ has angle $\theta \bmod 2 \pi$.

- Define

$$
p(a, \theta) \in \widetilde{C}_{0}^{(2-2 n)}(2 \pi n ; 0)
$$

to be the generator which wraps $n-1$ times around the 2-gon between $a$ and $b$, with edges at angles $\theta, \theta+\pi, \ldots \theta+(2 n-3) \pi$, all labeled ' $h$ ', in that order. Note that if $n=1$, then $p(a, \theta)$ is the constant path at $a$, while if $n>1$, then $p(a, \theta)$ has a kink parametrized by the interval $(\theta-3 \pi, \theta)$ and mapping to $a$. Also, $\delta p(a, \theta)=0$.

- Define

$$
e(a, \theta) \in \widetilde{C}_{1}^{(2-2 n)}(2 \pi n ; 0)
$$

to be the generator obtained from $Z_{n}(a, b)$, see Section 6.2, by relabeling the edge from $b$ to $a$ at angle $\theta-\pi$ by ' $e$ ' and ordering the $2 n-1$ ' $h$ ' edges counterclockwise. Then

$$
\delta e(a, \theta)=p(b, \theta+\pi)-p(a, \theta) .
$$


- Let

$$
q(a, b) \in \widetilde{C}_{1}^{(2-2 n)}(2 \pi n ; 0)
$$

be the sum of all $2 n$ generators that wrap $n$ times around the 2 -gon between $a$ and $b$ with $2 n-1$ edges labeled ' $h$ ' and ordered counterclockwise. That is,

$$
q(a, b):=\sum_{i=0}^{n-1}(e(a, \theta+2 i \pi)+e(b, \theta+(2 i+1) \pi)) .
$$

Note that $q(a, b)=q(b, a)$. By equation (70), $\delta q(a, b)=0$.

Lemma 8.6 (a) $\widetilde{H}_{0}^{(2-2 n)}(2 \pi n ; 0) \simeq \mathbb{Z}$ is generated by $p(a, \theta)$.

(b) $\widetilde{H}_{1}^{(2-2 n)}(2 \pi n ; 0) \simeq \mathbb{Z}$ is generated by $q(a, b)$.

Proof (a) This follows from the proof of Theorem 7.1(a), since by the symmetry in Lemma 6.20 we may assume that $a$ and $b$ are on the $x$-axis with $\theta=2 \pi$, and then the generator $p(a, \theta)$ is obtained by applying the splicing map $(n-1)$ times to a constant path which generates $\widetilde{H}_{0}^{(0)}(2 \pi ; 0)$.

(b) Let $\Lambda^{\prime}$ be the $n$-convex path that wraps $n$ times around the 2-gon with corners $a$ and $b$. Then $H_{1}^{(2-2 n)}\left(\Lambda^{\prime}\right) \simeq \mathbb{Z}$ is generated by $q(a, b)$. Indeed the summands in $q(a, b)$ are the only generators in $C_{1}^{(2-2 n)}\left(\Lambda^{\prime}\right)$, since such a generator must have $2 n-1$ ' $h$ ' edges, and their sum is the only cycle in $C_{1}^{(2-2 n)}\left(\Lambda^{\prime}\right)$ by equation (70). This cycle is not a boundary since $C_{2}^{(2-2 n)}\left(\Lambda^{\prime}\right)=0$, as the only generator in $C_{*}\left(\Lambda^{\prime}\right)$ with $2 n$ ' $h$ ' edges has index zero.

To finish the proof, we claim that the inclusion-induced map

$$
H_{1}^{(2-2 n)}\left(\Lambda^{\prime}\right) \rightarrow H_{1}^{(2-2 n)}(2 \pi n ; 0)
$$

is an isomorphism. This follows from the spectral sequence in the proof of Lemma 7.8. The reason is that in that spectral sequence, with $k$ and $m$ as in that proof, if $k \geq 3$ then there are no $m>0$ generators in the $E^{1}$ term in $C_{1}^{(2-2 n)}(\Lambda)$ or $C_{2}^{(2-2 n)}(\Lambda)$. Such a homology generator would have to be a sum of chain complex generators each having $2 n-1$ or $2 n$ ' $h$ ' edges, which means that it would be either a product of $n-1$ $Z_{1}$ 's and $1 H$ (which has index $2 k-3 \geq 3$ ), or a product of $n Z_{1}$ 's (which has index $0)$.

Lemma-Definition 8.7 There exists a unique assignment, to each triple $\left(a, \theta, \theta^{\prime}\right)$ with $a \in \mathbb{Z}^{2}$ and $\theta, \theta^{\prime} \in \mathbb{R}$ such that $\tan \theta, \tan \theta^{\prime} \in \mathbb{Q} \cup\{\infty\}$ and $\theta \leq \theta^{\prime}$, of an equivalence class of chains

$$
f\left(a, \theta, \theta^{\prime}\right) \in \frac{\widetilde{C}_{1}^{(2-2 n)}(2 \pi n ; 0)}{\operatorname{Im}(\delta)}
$$


such that:

(i) For each $\left(a, \theta, \theta^{\prime}\right)$ as above,

$$
\delta f\left(a, \theta, \theta^{\prime}\right)=p(a, \theta)-p\left(a, \theta^{\prime}\right) .
$$

(ii) Suppose that $0<\theta^{\prime}-\theta \leq \pi$, and that $b$ and $b^{\prime}$ are defined from $(a, \theta)$ and $\left(a, \theta^{\prime}\right)$ as in Definition 8.5. Then $f\left(a, \theta, \theta^{\prime}\right) \in C_{*}(\Lambda)$, where $\Lambda$ wraps $n-1$ times around the triangle with vertices $a, b, b^{\prime}$ (or 2-gon with vertices $b$ and $b^{\prime}$ when $\left.\theta^{\prime}-\theta=\pi\right)$ and has a kink at $a$ parametrized by the interval $\left(\theta^{\prime}-3 \pi, \theta\right)$.

(iii) If $\theta \leq \theta^{\prime} \leq \theta^{\prime \prime}$, then

$$
f\left(a, \theta, \theta^{\prime \prime}\right)=f\left(a, \theta, \theta^{\prime}\right)+f\left(a, \theta^{\prime}, \theta^{\prime \prime}\right) \bmod \operatorname{Im}(\delta) .
$$

Proof The proof has four steps.

Step 1 We first show that if $\theta^{\prime}-\theta<\pi$ and if $\left(a, b, b^{\prime}\right)$ is a simple triangle (see Definition 6.16), then there exists a chain $f\left(a, \theta, \theta^{\prime}\right)$ satisfying (i) and (ii). Reintroduce the notation from the proof of Lemma 6.17, with $c:=b^{\prime}$. Let $\mathcal{C}^{\prime}$ denote the set of subsets $I \subset\{1, \ldots, 3 n\}$ such that for distinct $i, j \in I$, the corners $c_{i}$ and $c_{j}$ of $\lambda$ are not adjacent, except that we allow at most one adjacent pair involving $c_{1}$ or $c_{3 n}$. For $I=\left\{i_{1}, \ldots, i_{k}\right\} \in \mathcal{C}^{\prime}$, let $T^{\prime}(I) \in C_{*}(\Lambda)$ be the generator with underlying path $\lambda(I)$ and with all edges labeled ' $h$ ', except that if $I$ does not contain an adjacent pair, then the edge that starts at $c_{3 n}$ and/or ends at $c_{1}$ is labeled ' $e$ '. Order the ' $h$ ' edges counterclockwise, starting at $c_{1}$ if $1 \notin I$, at $c_{2}$ if $1 \in I$ and $2 \notin I$, and at $c_{3}$ if $1,2 \in I$. Let $\theta_{i}$ denote the angle of the edge between corners $c_{i}$ and $c_{i+1}$. We may assume that $\theta=\theta_{1}$ and $\theta^{\prime}=\theta_{0}+\pi$. Then in this notation,

$$
\begin{aligned}
p\left(a, \theta_{1}\right) & =T^{\prime}(\{3,6, \ldots, 3 n-3,3 n-1,3 n\}), \\
p\left(a, \theta_{0}+\pi\right) & =T^{\prime}(\{2,5, \ldots, 3 n-4,3 n-1,3 n\}) .
\end{aligned}
$$

The differential of a generator $T^{\prime}(I)$ is given by

$$
\delta T^{\prime}(I)=-\sum_{I \cup\{i\} \in \mathcal{C}^{\prime}}(-1)^{\#\{j \notin I \mid i<j\}} T^{\prime}(I \cup\{i\}) .
$$

Now let $\mathcal{C}_{0}^{\prime}$ denote the set of $I=\left\{i_{1}, \ldots, i_{m}\right\} \in \mathcal{C}^{\prime}$ with $i_{1}<i_{2}<\cdots<i_{m}$ such that the $i_{k}$ 's alternate parity with $i_{1}$ odd. Define

$$
\begin{aligned}
f\left(a, \theta_{1}, \theta_{0}+\pi\right):= & \sum_{I=\left\{i_{1}, \ldots, i_{n-2}, 3 n-1,3 n\right\} \in \mathcal{C}_{0}^{\prime}} T^{\prime}(I) \\
= & T(\{3,6, \ldots, 3 n-9,3 n-6,3 n-1,3 n\}) \\
& +T(\{3,6, \ldots, 3 n-9,3 n-4,3 n-1,3 n\}) \\
& +\cdots+T(\{5,8, \ldots, 3 n-7,3 n-4,3 n-1,3 n\}) .
\end{aligned}
$$


Then it follows from equations (73) and (74) that this satisfies condition (i), ie

$$
\delta f\left(a, \theta_{1}, \theta_{0}+\pi\right)=p\left(a, \theta_{1}\right)-p\left(a, \theta_{0}+\pi\right) .
$$

Also, condition (ii) is satisfied since each term on the right side of (75) has the corners $c_{3 n-1}$ and $c_{3 n}$ rounded.

Step 2 We now show that if $0<\theta^{\prime}-\theta<\pi$, then a chain $f\left(a, \theta, \theta^{\prime}\right)$ satisfying (i) and (ii), if such exists, is unique modulo $\operatorname{Im}(\delta)$. The difference between any two such chains $f\left(a, \theta, \theta^{\prime}\right)$ is a cycle in $C_{1}^{(2-2 n)}(\Lambda)$. Thus it is enough to show that $H_{1}^{(2-2 n)}(\Lambda)=0$. Pick an angle $\phi$ with irrational tangent between $\theta^{\prime}-\pi$ and $\theta$. Proposition 6.11 shows that $H_{1}^{(2-2 n)}(\Lambda) \simeq H_{1}^{(2-2 n)}\left(C_{*}^{\phi}(\Lambda)\right)$. As in Lemma 6.12 and Example 6.10, $H_{1}^{(2-2 n)}\left(C_{*}^{\phi}(\Lambda)\right) \simeq H_{1}^{(2-2 n)}\left(\Lambda_{0}\right)$ where $\Lambda_{0}$ wraps $n-1$ times around a 2-gon and has a kink. But $C_{1}^{(2-2 n)}\left(\Lambda_{0}\right)=0$ because a generator of $C_{1}^{(2-2 n)}\left(\Lambda_{0}\right)$ would have $2 n-1$ edges labeled ' $h$ ', but generators of $C_{*}\left(\Lambda_{0}\right)$ have at most $2 n-2$ edges.

Step 3 We now show that there exists an assignment $f\left(a, \theta, \theta^{\prime}\right)$ satisfying (i), (ii), and (iii). Let $\left(a, \theta, \theta^{\prime}\right)$ be given. If $\theta=\theta^{\prime}$, define $f\left(a, \theta, \theta^{\prime}\right):=0$. Otherwise choose $\theta=\theta_{0}<\theta_{1}<\cdots<\theta_{k}=\theta^{\prime}$ such that $f\left(a, \theta_{i-1}, \theta_{i}\right)$ is defined by Step 1 for $i=1, \ldots, k$. Then define

$$
f\left(a, \theta, \theta^{\prime}\right):=\sum_{i=1}^{k} f\left(a, \theta_{i-1}, \theta_{i}\right) .
$$

As long as this is well-defined modulo $\operatorname{Im}(\delta)$, it clearly satisfies (i) and (ii) (by Step 1) and (iii) (by construction).

To show that (76) is well-defined modulo $\operatorname{Im}(\delta)$, let $\theta=\theta_{0}^{\prime}<\theta_{1}^{\prime}<\cdots<\theta_{k^{\prime}}^{\prime}=\theta^{\prime}$ be another set of choices to define $f\left(a, \theta, \theta^{\prime}\right)$. We need to show that

$$
\sum_{i=1}^{k} f\left(a, \theta_{i-1}, \theta_{i}\right)=\sum_{i=1}^{k^{\prime}} f\left(a, \theta_{i-1}^{\prime}, \theta_{i}^{\prime}\right) \bmod \operatorname{Im}(\delta) .
$$

Without loss of generality, $\theta_{1}<\theta_{1}^{\prime}$. By Step 2,

$$
f\left(a, \theta_{0}, \theta_{1}^{\prime}\right)=f\left(a, \theta_{0}, \theta_{1}\right)+f\left(a, \theta_{1}, \theta_{1}^{\prime}\right) \bmod \operatorname{Im}(\delta) .
$$

Subtracting (78) from (77), we see that to prove equation (77), it is enough to show that $f\left(a, \theta_{1}, \theta^{\prime}\right)$ is well defined modulo $\operatorname{Im}(\delta)$. We are now done by induction on $k+k^{\prime}$.

Step 4 An assignment $f\left(a, \theta, \theta^{\prime}\right)$ satisfying (i), (ii), and (iii) is unique modulo $\operatorname{Im}(\delta)$, because condition (iii) forces it to satisfy equation (76), and each summand on the right hand side of (76) is unique modulo $\operatorname{Im}(\delta)$ by conditions (i) and (ii) and Step 2. 
Lemma 8.8 If $(a, b, c)$ is a simple triangle, then with $\theta_{i}$ defined as above,

$$
\begin{aligned}
f\left(c, \theta_{3}, \theta_{2}+\pi\right) & +f\left(b, \theta_{2}, \theta_{1}+\pi\right)+f\left(a, \theta_{1}, \theta_{0}+\pi\right)= \\
& =-e\left(b, \theta_{2}\right)+e\left(a, \theta_{0}+\pi\right)-e\left(a, \theta_{1}\right) \bmod \operatorname{Im}(\delta) .
\end{aligned}
$$

Proof In the notation of the previous proof, define

$$
T_{k}^{\prime}:=\sum_{I=\left\{i_{1}, \ldots, i_{k}\right\} \in \mathcal{C}_{0}^{\prime}} T^{\prime}(I) .
$$

Equation (74) implies that

$$
\delta\left(-T_{n-1}^{\prime}\right)=T_{n}^{\prime}-T^{\prime}(\{2,5, \ldots, 3 n-1\})
$$

because on the right hand side, terms $T^{\prime}(I)$ in which the indices in $I$ do not alternate parity will appear twice with opposite signs or not at all, while terms $T^{\prime}(I)$ in which the indices do alternate parity will appear exactly once, and the only way to alternate parity starting with an even index is $2,5, \ldots, 3 n-1$. Now the right hand side of equation (81) equals

$$
\begin{gathered}
\left(\sum_{I=\left\{1,2, i_{3}, \ldots, i_{n}\right\} \in \mathcal{C}_{0}^{\prime}}+\sum_{I=\left\{1, i_{2}, \ldots, i_{n-1}, 3 n\right\} \in \mathcal{C}_{0}^{\prime}}+\sum_{I=\left\{i_{1}, \ldots, i_{n-2}, 3 n-1,3 n\right\} \in \mathcal{C}_{0}^{\prime}}\right) T^{\prime}(I)+ \\
+T^{\prime}(\{1,4, \ldots, 3 n-2\})-T^{\prime}(\{2,5, \ldots, 3 n-1\})+T^{\prime}(\{3,6, \ldots, 3 n\}) .
\end{gathered}
$$

These six terms equal the six terms in the relation (79).

Lemma 8.9 If $a, b$, and $\theta$ are as in Definition 8.5, then

$$
f(a, \theta, \theta+2 \pi)+e(a, \theta)+e(b, \theta+\pi)=q(a, b) \in \widetilde{H}_{1}^{(2-2 n)}(2 \pi n ; 0) .
$$

Proof Without loss of generality,

$$
a=\left(\begin{array}{l}
0 \\
0
\end{array}\right), \quad b=\left(\begin{array}{l}
1 \\
0
\end{array}\right), \quad \theta=0 .
$$

We can now do the entire calculation on the $x$-axis, ie in the subcomplex $C X_{*}(n)$, and use the notation of Section 7.1 to describe chains in this subcomplex. In this notation,

$$
\begin{aligned}
& e(a, 0)=\left(h_{b}^{a} h_{a}^{b}\right)^{n-1} e_{b}^{a} h_{a}^{b}, \\
& e(b, \pi)=\left(h_{b}^{a} h_{a}^{b}\right)^{n-1} h_{b}^{a} e_{a}^{b} .
\end{aligned}
$$

Let

$$
c:=\left(\begin{array}{c}
-1 \\
0
\end{array}\right)
$$


We can take

$$
\begin{aligned}
f(a, 0, \pi) & =\sum_{i=0}^{n-2}\left(h_{b}^{a} h_{a}^{b}\right)^{i} h_{b}^{c}\left(h_{c}^{a} h_{a}^{c}\right)^{n-2-i} h_{c}^{a} e_{a}^{a} h_{a}^{b}, \\
f(a, \pi, 2 \pi) & =\sum_{i=0}^{n-2} h_{a}^{c}\left(h_{c}^{a} h_{a}^{c}\right)^{i} h_{c}^{b}\left(h_{b}^{a} h_{a}^{b}\right)^{n-2-i} h_{b}^{a} e_{a}^{a},
\end{aligned}
$$

because the right hand side of each equation satisfies conditions (i) and (ii) in LemmaDefinition 8.7. By the definition of $\delta$ and straightforward manipulation of sums,

$$
\begin{aligned}
\delta\left(\sum_{i=0}^{n-2} \sum_{j=0}^{n-2-i}\left(h_{b}^{a} h_{a}^{b}\right)^{i} h_{b}^{c}\left(h_{c}^{a} h_{a}^{c}\right)^{j} h_{c}^{b}\left(h_{b}^{a} h_{a}^{b}\right)^{n-1-i-j}\right) & = \\
-f(a, 0, \pi)-f(a, \pi, 2 \pi) & -e(a, 0)-e(b, \pi)+q(a, b),
\end{aligned}
$$

which completes the proof.

Proof of Lemma 8.1 Since this lemma is computing a differential in the universal coefficient spectral sequence, it is enough to verify the conclusion of the lemma for a single choice of $p, s$, and $t$. Introduce the lattice points $a:=(0,0), b:=(1,0)$, $c:=(2,0), d:=(0,1), e:=(1,1)$, and $f:=(2,1)$. By Lemma 8.6(a), we can take

$$
p:=p(a, 0) .
$$

By equations (70) and (71), we can take

$$
\begin{aligned}
s & :=f(b, 0, \pi)+e(a, 0), \\
t & :=-f(a, 0, \pi / 2)-e(d,-\pi / 2)-f(d,-\pi / 2,0) .
\end{aligned}
$$

These chains are only defined $\bmod \operatorname{Im}(\delta)$, which is fine here since we just need to evaluate the homology class of $(y-1) s-(x-1) t$. By definition,

$$
\begin{aligned}
(y-1) s-(x-1) t= & f(b, 0, \pi / 2)+e(e,-\pi / 2)+f(e,-\pi / 2,0) \\
& -f(a, 0, \pi / 2)-e(d,-\pi / 2)-f(d,-\pi / 2,0) \\
& +f(e, 0, \pi)+e(d, 0)-f(b, 0, \pi)-e(a, 0) .
\end{aligned}
$$

By Lemma 8.8,

$$
\begin{aligned}
f(b, 3 \pi / 4, \pi) & +f(a, 0, \pi / 2)+f(d,-\pi / 2,-\pi / 4)= \\
& =-e(a, 0)+e(d,-\pi / 4)-e(d,-\pi / 2), \\
f(b, \pi / 2,3 \pi / 4) & +f(d,-\pi / 4,0)+f(e,-\pi,-\pi / 2)= \\
& =-e(d,-\pi / 4)+e(e,-\pi / 2)-e(e,-\pi) .
\end{aligned}
$$


Putting these two six-term relations into equation (82) and repeatedly applying the relation (72) gives

$$
(y-1) s-(x-1) t=f(e,-\pi, \pi)+e(e,-\pi)+e(d, 0) .
$$

By Lemmas 8.9 and $8.6\left(\right.$ b), this generates $\widetilde{H}_{1}^{(2-2 n)}(2 \pi n ; 0) \simeq \mathbb{Z}$.

Lemma 8.10 Let $(a, b, c)$ be a simple triangle, and let $\theta_{1}, \ldots, \theta_{3 n}$ be defined as previously. Then there exists a chain

$$
r(a, b, c) \in \widetilde{C}_{2}^{(2-2 n)}(2 \pi n ; 0)
$$

such that if $U$ is defined using $\theta$, then

$$
\begin{aligned}
& \delta r(a, b, c)=q(a, b)-q(a, c), \\
& U r(a, b, c)=\left\{\begin{array}{cl}
-Z_{n}(a, b), & \theta \in\left(\theta_{3 i+1}-\pi, \theta_{3 i}\right), \\
Z_{n}(b, c), & \theta \in\left(\theta_{3 i}, \theta_{3 i+1}\right), \\
-Z_{n}(c, a), & \theta \in\left(\theta_{3 i+1}, \theta_{3 i}+\pi\right), \\
0, & \text { otherwise. }
\end{array}\right.
\end{aligned}
$$

Proof Suppose first that

$$
\theta \notin\left(\theta_{3 i}, \theta_{3 i+2}-\pi\right),\left(\theta_{3 i+1}, \theta_{3 i}+\pi\right), \quad i=0, \ldots, n-1 .
$$

Let $\Lambda$ be the $n$-convex path that wraps $n$ times around the triangle $(a, b, c)$. There is an obvious action of the cyclic group $\mathbb{Z} / 3 n$ on the chain complex $C_{*}(\Lambda)$, given by a chain map

$$
\eta: C_{*}(\Lambda) \rightarrow C_{*}(\Lambda)
$$

which rotates everything counterclockwise, replacing $\theta_{i}$ by $\theta_{i+1}$, etc. Define

$$
r(a, b, c):=(\eta-1) \sum_{i=0}^{n-1} \eta^{i} T_{n-1}^{\prime}
$$

where $T_{n-1}^{\prime}$ is defined in equation (80). We saw in the proof of Lemma 8.8 that

$$
\begin{aligned}
\delta\left(-T_{n-1}^{\prime}\right)= & f\left(c, \theta_{3}, \theta_{2}+\pi\right)+f\left(b, \theta_{2}, \theta_{1}+\pi\right)+f\left(a, \theta_{1}, \theta_{0}+\pi\right)+ \\
& +e\left(b, \theta_{2}\right)-e\left(a, \theta_{0}+\pi\right)+e\left(a, \theta_{1}\right) .
\end{aligned}
$$


In $\delta r(a, b, c)$, all the $f$ 's cancel and

$$
\begin{aligned}
\delta r(a, b, c)= & \sum_{i=0}^{n-1}\left(e\left(a, \theta_{1}+2 \pi i\right)+e\left(b, \theta_{1}+\pi+2 \pi i\right)-\right. \\
& \left.\quad-e\left(a, \theta_{0}+\pi+2 \pi i\right)-e\left(c, \theta_{0}+2 \pi+2 \pi i\right)\right) \\
= & q(a, b)-q(a, c) .
\end{aligned}
$$

This proves (83). To prove (84), observe from the definition of $U$ that

$$
U T^{\prime}(I)=\left\{\begin{array}{cll}
T(\{1\} \cup I), & 3 n, 1,2 \notin I ; & \theta \in\left(\theta_{0}, \theta_{2}-\pi\right), \\
T(I \cup\{3 n\}), & 3 n-1,3 n, 1 \notin I ; & \theta \in\left(\theta_{1}-\pi, \theta_{0}\right), \\
0, & \text { otherwise. }
\end{array}\right.
$$

In particular,

$$
U T_{n-1}^{\prime}=\left\{\begin{array}{cl}
T(\{3,6, \ldots, 3 n\}), & \theta \in\left(\theta_{1}-\pi, \theta_{0}\right), \\
0, & \theta \in\left(\theta_{2}-\pi, \theta_{3 n+1}-\pi\right) .
\end{array}\right.
$$

Note that $U T_{n-1}^{\prime}$ is more complicated when $\theta \in\left(\theta_{0}, \theta_{2}-\pi\right)$. But under our assumption (85) on $\theta$, we do not have to consider that case, and the above gives equation (84).

If the assumption (85) does not hold, then redefine $T_{n-1}^{\prime}$ by summing over sequences that start with an even index instead of an odd one. Then (87) holds with a minus sign. If we redefine $r(a, b, c)$ with a minus sign in equation (86), then the rest of the argument goes through.

Proof of Lemma 8.4 Let $a, b, c, d, e, f$ be the lattice points in the proof of Lemma 8.1. By Lemma $8.6(\mathrm{~b}), \widetilde{H}_{1}^{(2-2 n)}(2 \pi n ; 0)$ is generated by

$$
q_{0}:=q(a, b) .
$$

By equation (83), the requirement (65) is satisfied by

$$
\begin{aligned}
& u_{1}:=r(b, c, e)+r(b, e, a), \\
& v_{1}:=-r(a, b, d)-r(d, a, e) .
\end{aligned}
$$

Choose $\theta \in(\pi / 2, \pi)$. Then by equation (84),

$$
\begin{aligned}
U r(b, c, e) & =0, \\
U r(b, e, a) & =-Z_{n}(a, b)=Z_{n}(b, a), \\
U r(a, b, d) & =0, \\
U r(d, a, e) & =-Z_{n}(d, a)=Z_{n}(a, d),
\end{aligned}
$$

so (66) holds as well. 


\section{Axioms for the chain complex}

In this section we prove that the chain complex $\left(\widetilde{C}_{*}(2 \pi n ; \Gamma), \delta\right)$ defined in Section 3.1 is characterized by certain axioms. This will be used in Section 11 to relate the chain complex to the embedded contact homology of $T^{3}$. It will simplify some arguments below to consider all $\Gamma$ at once, so introduce the notation

$$
\widetilde{C}_{*}(2 \pi n):=\bigoplus_{\Gamma \in \mathbb{Z}^{2}} \widetilde{C}_{*}(2 \pi n ; \Gamma) .
$$

\subsection{The axioms}

Fix a positive integer $n$. We now list a series of axioms for a chain complex $\left(C_{*}, \partial\right)$ over $\mathbb{Z}\left[\mathbb{Z}^{2}\right]$.

I (Generators) $C_{*}=\widetilde{C}_{*}(2 \pi n)$ as a $\mathbb{Z}\left[\mathbb{Z}^{2}\right]$-module.

II (Index) $\partial$ respects the decomposition (88) and has degree -1 with respect to the relative grading $I$ on $\widetilde{C}_{*}(2 \pi n ; \Gamma)$ defined in Section 3.1.

To state the next axioms, let $\alpha$ and $\beta$ be generators of $C_{*}$ with the same period $\Gamma$ and with underlying admissible paths $\lambda$ and $\mu$. By an "edge" of $\alpha$ or $\beta$, we mean an edge of the corresponding admissible path $\lambda$ or $\mu$. We write $\beta \leq \alpha$ if $\mu \leq \lambda$, see Section 2.3 .

We say that two edges of $\alpha$ and $\beta$ "agree" (resp. "partially agree") if they correspond to the same angle $\theta \in \mathbb{R} / 2 \pi n \mathbb{Z}$, and if their adjacent corners map to the same points in $\mathbb{Z}^{2}$ (resp. to points on the same line in $\mathbb{R}^{2}$ ) (for a given lift of $\theta$ to $\mathbb{R}$ when $\Gamma \neq 0$ ).

Let $D(\alpha, \beta)$ denote the closure of the set of all $t \in \mathbb{R} / 2 \pi n \mathbb{Z}$ such that $\lambda(t)$ and $\mu(t)$ are defined and unequal (for a given lift of $t$ to $\mathbb{R}$ when $\Gamma \neq 0$ ). Note that a point $\theta \in \mathbb{R} / 2 \pi n \mathbb{Z}$ corresponding to an edge of $\alpha$ (resp. $\beta$ ) is in $D(\alpha, \beta)$ if and only if this edge does not agree with any edge of $\beta$ (resp. $\alpha$ ).

III (Nesting) If $\langle\partial \alpha, \beta\rangle \neq 0$, then $\beta \leq \alpha$.

IV (Label Matching) Suppose that $\langle\partial \alpha, \beta\rangle \neq 0$. If two edges of $\alpha$ and $\beta$ agree, then the labels (' $e$ ' or ' $h$ ') of the two edges are the same. If two edges of $\alpha$ and $\beta$ partially agree, and if the edge of $\beta$ is labeled ' $h$ ', then the edge of $\alpha$ is also labeled ' $h$ '.

$\mathrm{V}$ (Connectedness) If $\langle\partial \alpha, \beta\rangle \neq 0$ then the set $D(\alpha, \beta)$ is connected.

VI (No Double Rounding) If $\langle\partial \alpha, \beta\rangle \neq 0$ then $\alpha$ cannot have three edges in $D(\alpha, \beta)$. 
The next axiom says essentially that the differential coefficient $\langle\partial \alpha, \beta\rangle$ depends only on the local change needed to get from $\alpha$ to $\beta$. To state it, suppose that $\alpha$ and $\beta$ satisfy the Nesting and Label Matching conditions above. We construct generators $\alpha^{\prime}$ and $\beta^{\prime}$ as follows. If two edges of $\alpha$ and $\beta$ agree, remove them both. If two edges of $\alpha$ and $\beta$ partially agree (which by Nesting implies that the edge of $\beta$ has smaller multiplicity than the edge of $\alpha$ ), remove the edge of $\beta$ and shorten the edge of $\alpha$ by the same amount, while preserving the number of ' $h$ ' labels. (That is, if the edge of $\alpha$ is labeled ' $h$ ' and the edge of $\beta$ is labeled ' $e$ ', then the edge of $\alpha$ ' is labeled ' $h$ '; otherwise the edge of $\alpha^{\prime}$ is labeled ' $e$ '.) In particular the multiplicity functions of the underlying admissible paths $\lambda, \mu, \lambda^{\prime}, \mu^{\prime}$ of $\alpha, \beta, \alpha^{\prime}, \beta^{\prime}$ respectively satisfy

$$
m_{\alpha}-m_{\alpha^{\prime}}=m_{\beta}-m_{\beta^{\prime}}
$$

So far we have only defined $\lambda^{\prime}$ and $\mu^{\prime}$ up to translation, but there is a unique choice of $\lambda^{\prime}$ and $\mu^{\prime}$, up to simultaneous translation of both, such that

$$
\lambda(t)-\mu(t)=\lambda^{\prime}(t)-\mu^{\prime}(t)
$$

for all $t$ not an edge. We order the edges of $\alpha$ and $\beta$ such that the agreeing ' $h$ ' edges are ordered first and in the same order, and this determines an ordering of the ' $h$ ' edges of $\alpha^{\prime}$ and $\beta^{\prime}$. Note for future reference that

$$
I(\alpha, \beta)=I\left(\alpha^{\prime}, \beta^{\prime}\right)
$$

VII (Locality) Let $\alpha$ and $\beta$ satisfy the Nesting and Label Matching conditions, and let $\alpha^{\prime}$ and $\beta^{\prime}$ be obtained from $\alpha$ and $\beta$ by removing matching edges as above. Then

$$
\langle\partial \alpha, \beta\rangle=\left\langle\partial \alpha^{\prime}, \beta^{\prime}\right\rangle
$$

Suppose that $\beta$ is obtained from $\alpha$ by rounding a corner and locally losing one ' $h$ ', ie the differential coefficient $\langle\delta \alpha, \beta\rangle= \pm 1$ as in Section 3.1. If furthermore only one new edge is created by the rounding process, and if this edge has multiplicity one (ie the corresponding segment in $\mathbb{Z}^{2}$ contains no interior lattice points), then we say that $\beta$ is obtained from $\alpha$ by simple rounding. If no edges at all are created by the rounding process, ie if $\alpha$ turns by angle $\pi$ at the rounded corner, then we say that $\beta$ is obtained from $\alpha$ by degenerate rounding.

VIII (Simple Rounding) If $\beta$ is obtained from $\alpha$ by simple rounding, then $\langle\partial \alpha, \beta\rangle=$ $\langle\delta \alpha, \beta\rangle$.

IX (Degenerate Rounding) If $\beta$ is obtained from $\alpha$ by degenerate rounding then $\langle\partial \alpha, \beta\rangle=\langle\delta \alpha, \beta\rangle$. 


\subsection{Uniqueness of the chain complex}

As usual let $\delta$ denote the differential on $\widetilde{C}_{*}(2 \pi n)$ defined in Section 3.1. Of course, $\left(\widetilde{C}_{*}(2 \pi n), \delta\right)$ satisfies the above axioms.

Proposition 9.1 Let $\partial$ be a differential on $\widetilde{C}_{*}(2 \pi n)$ satisfying the axioms of Section 9.1. Then $\partial=\delta$.

Proof Throughout this proof, $\alpha$ and $\beta$ will denote generators of $\widetilde{C}_{*}(2 \pi n)$, and $\lambda$ and $\mu$ will denote their underlying admissible paths.

Lemma 9.2 If $\langle\partial \alpha, \beta\rangle \neq 0$, then $\langle\delta \alpha, \beta\rangle \neq 0$.

Proof Suppose that $\langle\partial \alpha, \beta\rangle \neq 0$. By the Nesting axiom, $\beta \leq \alpha$. By Proposition 2.13, the polygon $\mu$ can be obtained from $\lambda$ by a sequence of $k$ corner roundings for some nonnegative integer $k$. By Lemma 3.9,

$$
I(\alpha, \beta)=2 k-\# h(\alpha)+\# h(\beta) .
$$

Let $D:=D(\alpha, \beta)$ be defined as in Section 9.1. Let $l$ denote the number of edges of $\alpha$ that are in $D$, ie that do not agree with any edges in $\beta$. Since $D$ is connected by the Connectedness axiom, these $l$ edges of $\alpha$ are consecutive. Observe that

$$
l \leq k+1 \text {. }
$$

Otherwise, at least one of the corners between the edges of $\alpha$ in $D$ is not rounded in a sequence of $k$ roundings from $\mu$ to $\lambda$. If $D \neq \mathbb{R} / 2 \pi n \mathbb{Z}$, then $D$ is separated by such a corner, contradicting the Connectedness axiom. If $D=\mathbb{R} / 2 \pi n \mathbb{Z}$, then two corners in $\alpha$ are not rounded and these two corners separate $D$.

By the Label Matching axiom, we can calculate $\# h(\alpha)-\# h(\beta)$ by considering only the edges of $\alpha$ and $\beta$ in $D$, so

$$
\# h(\alpha)-\# h(\beta) \leq l .
$$

Combining this with the previous inequality and equation gives

$$
I(\alpha, \beta) \geq k-1 \text {. }
$$

By the Index axiom, $I(\alpha, \beta)=1$, so the only possibilities are $k=0, k=1$, or $k=2$.

The case $k=0$ is impossible, as then the Label Matching axiom would imply that $\alpha=\beta$ so that $I(\alpha, \beta)=0$. 
If $k=2$ then equality must hold in the above inequalities so $l=3$. But this is forbidden by the No Double Rounding axiom.

Therefore $k=1$, so $\mu$ is obtained from $\lambda$ by rounding a corner. By the index formula, $\# h(\alpha)-\# h(\beta)=1$. Together with the Label Matching axiom, this implies that $\langle\delta \alpha, \beta\rangle= \pm 1$.

Lemma 9.3 If $\langle\delta \alpha, \beta\rangle \neq 0$, then

$$
\langle\partial \alpha, \beta\rangle=\langle\delta \alpha, \beta\rangle .
$$

Proof Suppose that $\langle\delta \alpha, \beta\rangle \neq 0$; we will show that (90) holds. The strategy is to use $\partial^{2}=0$ to solve for the unknown differential coefficients. The following is similar to an argument in our earlier paper [10, Section 3.8], but because we are considering more general polygonal paths here we can make some simplifications.

We know that $\beta$ is obtained from $\alpha$ by rounding a corner $c$ and locally losing one ' $h$ '. Let $\theta_{1}$ and $\theta_{2}$ be the edges of $\alpha$ preceding and following $c$, respectively. Let

$$
\Delta:=\operatorname{det}\left(\begin{array}{ll}
x_{\theta_{1}} & x_{\theta_{2}} \\
y_{\theta_{1}} & y_{\theta_{2}}
\end{array}\right) \in \mathbb{Z} .
$$

By the definition of rounding a corner, $\theta_{2}-\theta_{1} \in(0, \pi]$, and in particular $\Delta \geq 0$. We will now prove equation (90) by induction on $\Delta$.

If $\Delta=0$, then (90) holds by the Degenerate Rounding axiom.

If $\Delta=1$, then the triangle with vertices

$$
\lambda(c)-\left(\begin{array}{c}
x_{\theta_{1}} \\
y_{\theta_{1}}
\end{array}\right), \quad \lambda(c), \quad \lambda(c)+\left(\begin{array}{l}
x_{\theta_{2}} \\
y_{\theta_{2}}
\end{array}\right)
$$

is simple, so (90) holds by the Simple Rounding axiom.

Now suppose that $\Delta>1$, and assume that the lemma holds for all smaller values of $\Delta$. Let $c^{\prime}$ be the corner of $\alpha$ following the edge $\theta_{2}$. Let $\theta_{3}$ be the edge of $\beta$ preceding $c^{\prime}$, ie the last edge of $\beta$ created by rounding at $c$. By the Locality axiom, we may replace $\alpha$ and $\beta$ by the generators $\alpha^{\prime}$ and $\beta^{\prime}$ in the statement of the Locality axiom, and then add one new edge to each, to arrange the following:

- The edges $\theta_{1}$ and $\theta_{2}$ of $\alpha$ have multiplicity 1 .

- $\alpha$ has only one edge other than $\theta_{1}$ and $\theta_{2}$, and $\beta$ has only one edge other than the edges created by rounding the corner $c$. In both $\alpha$ and $\beta$, this additional edge is at angle $\theta_{3}+\pi$ with multiplicity 1 . 
- If the edge $\theta_{3}$ of $\beta$ is labeled ' $h$ ', then the edge $\theta_{3}+\pi$ of $\alpha$ and $\beta$ is labeled ' $e$ '; otherwise the edge $\theta_{3}+\pi$ of $\alpha$ and $\beta$ is labeled ' $h$ '.

The last condition above ensures that it is possible to round the corner $c^{\prime}$ of $\beta$ and locally lose one ' $h$ ' to obtain a well-defined (up to sign) generator $\gamma$ with $\langle\delta \beta, \gamma\rangle \neq 0$. Since this rounding is degenerate, we also know that

$$
\langle\partial \beta, \gamma\rangle=\langle\delta \beta, \gamma\rangle \neq 0 .
$$

An example of the admissible paths underlying $\alpha$ and $\gamma$ is shown below.

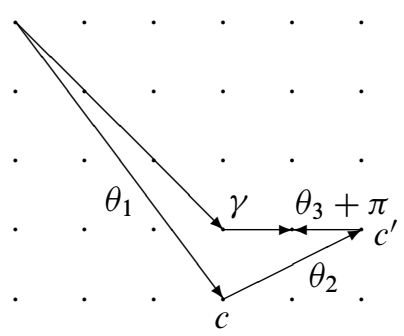

In general the rounding of $\alpha$ at $c^{\prime}$ is simple, because the triangle with vertices

$$
\lambda(c), \quad \lambda\left(c^{\prime}\right), \quad \lambda\left(c^{\prime}\right)-\left(\begin{array}{l}
x_{\theta_{3}} \\
y_{\theta_{3}}
\end{array}\right)
$$

is simple, by the definition of rounding the corner of $\alpha$ at $c$. Thus there is a unique (up to sign) generator $\beta^{\prime}$ obtained from $\alpha$ by rounding the corner at $c^{\prime}$ and locally losing one ' $h$ '. This generator $\beta^{\prime}$ satisfies $\left\langle\delta \alpha, \beta^{\prime}\right\rangle \neq 0$, and because $\beta^{\prime}$ is obtained from $\alpha$ by simple rounding, we also know that

$$
\left\langle\partial \alpha, \beta^{\prime}\right\rangle=\left\langle\delta \alpha, \beta^{\prime}\right\rangle \neq 0 .
$$

Finally, the edge labels work out so that we can round $\beta^{\prime}$ at $c$ and locally lose one ' $h$ ' to obtain $\gamma$, and in particular $\left\langle\delta \beta^{\prime}, \gamma\right\rangle \neq 0$. Moreover the determinant corresponding to this rounding as in (91) is less than $\Delta$, because the triangle with vertices

$$
\lambda(c)-\left(\begin{array}{l}
x_{\theta_{1}} \\
y_{\theta_{1}}
\end{array}\right), \quad \lambda(c), \quad \lambda\left(c^{\prime}\right)-\left(\begin{array}{l}
x_{\theta_{3}} \\
y_{\theta_{3}}
\end{array}\right)
$$

is a proper subset of the triangle with vertices

$$
\lambda(c)-\left(\begin{array}{c}
x_{\theta_{1}} \\
y_{\theta_{1}}
\end{array}\right), \quad \lambda(c), \quad \lambda\left(c^{\prime}\right) .
$$


So by inductive hypothesis,

$$
\left\langle\partial \beta^{\prime}, \gamma\right\rangle=\left\langle\delta \beta^{\prime}, \gamma\right\rangle \neq 0
$$

By Lemmas 3.12(a) and 9.2, there does not exist a generator $\beta^{\prime \prime}$, other than $\pm \beta^{\prime}$ and possibly $\pm \beta$, with $\left\langle\partial \alpha, \beta^{\prime \prime}\right\rangle,\left\langle\partial \beta^{\prime \prime}, \gamma\right\rangle \neq 0$. Using this fact and then plugging in the previous three equations, we get

$$
\begin{aligned}
0=\left\langle\partial^{2} \alpha, \gamma\right\rangle & =\langle\partial \alpha, \beta\rangle\langle\partial \beta, \gamma\rangle+\left\langle\partial \alpha, \beta^{\prime}\right\rangle\left\langle\partial \beta^{\prime}, \gamma\right\rangle \\
& =\langle\partial \alpha, \beta\rangle\langle\delta \beta, \gamma\rangle+\left\langle\delta \alpha, \beta^{\prime}\right\rangle\left\langle\delta \beta^{\prime}, \gamma\right\rangle .
\end{aligned}
$$

By Lemma 3.12(b),

$$
0=\left\langle\delta^{2} \alpha, \gamma\right\rangle=\langle\delta \alpha, \beta\rangle\langle\delta \beta, \gamma\rangle+\left\langle\delta \alpha, \beta^{\prime}\right\rangle\left\langle\delta \beta^{\prime}, \gamma\right\rangle .
$$

Since all factors on the right hand side are nonzero, comparing this equation with the previous one proves (90).

The above two lemmas prove Proposition 9.1.

\section{$10 \quad J$-holomorphic curves in $\mathbb{R} \times T^{3}$}

Having completed the proofs of our algebraic theorems, we now gather some fundamental facts about $J$-holomorphic curves in $\mathbb{R} \times T^{3}$, in preparation for computing the embedded contact homology of $T^{3}$. Section 10.1 gives basic definitions. In Section 10.2 we establish a dictionary between some of the combinatorics of Section 2 and the geometry of $J$-holomorphic curves in $\mathbb{R} \times T^{3}$. In Section 10.3 we prove a useful restriction on the latter in terms of the partial order from Section 2.3. In Section 10.4 and Section 10.5 we recall and prove some basic classification results for $J$-holomorphic curves in $\mathbb{R} \times T^{3}$.

\section{1 $J$-holomorphic curves in symplectizations}

Let $Y$ be a closed oriented 3-manifold with a contact form $\lambda$; see Section 1.1 for the basic contact terminology.

Definition 10.1 An orbit set is a finite set of pairs $\alpha=\left\{\left(\alpha_{i}, m_{i}\right)\right\}$ where the $\alpha_{i}$ 's are distinct embedded Reeb orbits and the $m_{i}$ 's are positive integers ("multiplicities"). The homology class of $\alpha$ is defined by

$$
[\alpha]:=\sum_{i} m_{i}\left[\alpha_{i}\right] \in H_{1}(Y) .
$$


Definition 10.2 If $\alpha=\left\{\left(\alpha_{i}, m_{i}\right)\right\}$ and $\beta=\left\{\left(\beta_{j}, n_{j}\right)\right\}$ are orbit sets with $[\alpha]=[\beta]$, let $H_{2}(Y, \alpha, \beta)$ denote the set of relative homology classes of 2-chains $Z$ in $Y$ with

$$
\partial Z=\sum_{i} m_{i} \alpha_{i}-\sum_{j} n_{j} \beta_{j}
$$

Thus $H_{2}(Y, \alpha, \beta)$ is an affine space modelled on $H_{2}(Y)$.

Definition 10.3 An almost complex structure $J$ on $\mathbb{R} \times Y$ is admissible if $J$ is $\mathbb{R}$ invariant; $J(\xi)=\xi$ with $d \lambda(v, J v)>0$ for nonzero $v \in \xi$; and $J\left(\partial_{s}\right)$ is a positive multiple of $R$, where $s$ denotes the $\mathbb{R}$ coordinate.

For our purposes, a $J$-holomorphic curve is a nonconstant map $u: C \rightarrow \mathbb{R} \times Y$, modulo reparametrization, where $C$ is a punctured compact (possibly disconnected) Riemann surface with a complex structure $j$, such that $d u \circ j=J \circ d u$. When $u$ is an embedding, we often identify $u$ with its image in $\mathbb{R} \times Y$.

For admissible $J$, if $\gamma \subset Y$ is an embedded Reeb orbit, then $\mathbb{R} \times \gamma \subset \mathbb{R} \times Y$ is a $J$-holomorphic cylinder, which we call a trivial cylinder. Given a more general $J$-holomorphic curve $u: C \rightarrow \mathbb{R} \times Y$, a positive end at $\gamma$ of multiplicity $k$ is an end of $u$ asymptotic to $\mathbb{R} \times \gamma^{k}$ as $s \rightarrow+\infty$, where $\gamma^{k}$ denotes the $k$-fold connected covering of $\gamma$. A negative end is defined analogously with $s \rightarrow-\infty$.

Definition 10.4 If $\alpha=\left\{\left(\alpha_{i}, m_{i}\right)\right\}$ and $\beta=\left\{\left(\beta_{j}, n_{j}\right)\right\}$ are orbit sets with $[\alpha]=[\beta]$, let $\mathcal{M}^{J}(\alpha, \beta)$ denote the moduli space of $J$-holomorphic curves $u: C \rightarrow \mathbb{R} \times Y$ as above such that:

- $u$ has positive ends at $\alpha_{i}$, whose multiplicities sum to $m_{i}$.

- Similarly $u$ has negative ends at $\beta_{j}$ of total multiplicity $n_{j}$.

- $u$ has no other ends.

Note that $\mathbb{R}$ acts on $\mathcal{M}^{J}(\alpha, \beta)$ by translation in the $\mathbb{R}$ direction in $\mathbb{R} \times Y$. If $u \in$ $\mathcal{M}^{J}(\alpha, \beta)$, then the projection of $u$ from $\mathbb{R} \times Y$ to $Y$ has a well-defined relative homology class

$$
[u] \in H_{2}(Y, \alpha, \beta) .
$$

Definition 10.5 If $Z \in H_{2}(Y, \alpha, \beta)$, let

$$
\mathcal{M}^{J}(\alpha, \beta ; Z):=\left\{u \in \mathcal{M}^{J}(\alpha, \beta) \mid[u]=Z\right\} .
$$


Definition 10.6 If $\alpha=\left\{\left(\alpha_{i}, m_{i}\right)\right\}$ is an orbit set, define the symplectic action

$$
\mathcal{A}(\alpha):=\sum_{i} m_{i} \int_{\alpha_{i}} \lambda
$$

Lemma 10.7 For an admissible almost complex structure $J$, if $\mathcal{M}^{J}(\alpha, \beta)$ is nonempty, then:

(a) $\mathcal{A}(\alpha) \geq \mathcal{A}(\beta)$.

(b) If $\mathcal{A}(\alpha)=\mathcal{A}(\beta)$, then $\alpha=\beta$ and every element of $\mathcal{M}^{J}(\alpha, \beta)$ maps to a union of trivial cylinders.

Proof Suppose $u \in \mathcal{M}^{J}(\alpha, \beta)$. Admissibility of $J$ implies that if $v$ is a tangent vector to a point in the domain $(C, j)$, then $u^{*} d \lambda(v, j v) \geq 0$. Part (a) follows immediately from Stokes theorem. Part (b) holds because $u^{*} d \lambda(v, j v)=0$ only if $d u$ sends $v$ to the span of $\partial_{s}$ and $R$ in $T(\mathbb{R} \times Y)$.

\subsection{Admissible paths and orbit sets in $T^{3}$}

Fix a positive integer $n$. We now specialize to the example $Y=T^{3}$ with the contact form $\lambda_{n}$ defined by (4) and (5). The Reeb orbits of $\lambda_{n}$ consist of circles of Reeb orbits at each $\theta \in \Theta_{n}$, where

$$
\Theta_{n}:=\{\theta \in \mathbb{R} / 2 \pi n \mathbb{Z} \mid \tan \theta \in \mathbb{Q} \cup\{\infty\}\} .
$$

Each Reeb orbit $\gamma$ in the circle at $\theta$ has homology class

$$
[\gamma]=\left(0,\left(x_{\theta}, y_{\theta}\right)\right) \in H_{1}\left(T^{3}\right) .
$$

In this setting we define a Morse-Bott orbit set to be a finite set of pairs $\alpha=\left\{\left(\alpha_{i}, m_{i}\right)\right\}$ where each $\alpha_{i}$ is a component of the space of embedded Reeb orbits and each $m_{i}$ is a positive integer. A Morse-Bott orbit set $\alpha$ with $[\alpha]=\Gamma \in \mathbb{Z}^{2}=H_{1}\left(T^{2}\right) \subset H_{1}\left(T^{3}\right)$ is equivalent to a multiplicity function

$$
m: \Theta_{n} \longrightarrow \mathbb{Z}_{\geq 0}
$$

which is finitely supported and which satisfies

$$
\sum_{\theta \in \Theta_{n}} m(\theta)\left(\begin{array}{l}
x_{\theta} \\
y_{\theta}
\end{array}\right)=\Gamma .
$$

So by the discussion in Section 2.1, there is a canonical bijection

$$
\left\{\begin{array}{c}
\text { Morse-Bott orbit } \\
\text { sets } \alpha \text { with }[\alpha]=\Gamma
\end{array}\right\}=\left\{\begin{array}{c}
\text { periodic admissible paths of } \\
\text { rotation number } n \text { and period } \Gamma
\end{array}\right\} / \text { translation. }
$$


Remark 10.8 Under this correspondence, the length of a periodic admissible path, defined in Section 2.3, agrees with the symplectic action of the corresponding MorseBott orbit set as in Definition 10.6.

There seems to be no natural way to resolve the translation ambiguity in (93) for a single path. However, the relative translation ambiguity of a pair of paths does have a geometric interpretation, as we now explain.

Definition 10.9 Let $m$ and $m^{\prime}$ be finitely supported functions $\Theta_{n} \rightarrow \mathbb{Z}_{\geq 0}$ satisfying (92). A relative placement of $m$ and $m^{\prime}$ is a locally constant map

$$
f:(\mathbb{R} / 2 \pi n \mathbb{Z}) \backslash\left(\operatorname{supp}(m) \cup \operatorname{supp}\left(m^{\prime}\right)\right) \longrightarrow \mathbb{Z}^{2}
$$

satisfying the "jumping condition"

$$
\frac{d f(t)}{d t}=\left(m(t)-m^{\prime}(t)\right) \sum_{\theta \in \Theta_{n}}\left(\begin{array}{l}
x_{\theta} \\
y_{\theta}
\end{array}\right) \delta_{\theta}(t) .
$$

Let $\mathcal{R}\left(m, m^{\prime}\right)$ denote the set of all such $f$; this is an affine space over $\mathbb{Z}^{2}$.

The significance of this definition is that if $\Lambda$ and $\Lambda^{\prime}$ are periodic admissible paths of rotation number $n$ and period $\Gamma$ with multiplicity functions $m$ and $m^{\prime}$ respectively, then

$$
f=\Lambda-\Lambda^{\prime}
$$

is a relative placement of $m$ and $m^{\prime}$.

On the geometric side, if $\alpha$ and $\alpha^{\prime}$ are Morse-Bott orbit sets with $[\alpha]=\left[\alpha^{\prime}\right]$, then $H_{2}\left(T^{3}, \alpha, \alpha^{\prime}\right)$ is a well-defined affine space over $H_{2}\left(T^{3}\right) / H_{2}\left(T^{2}\right)=\mathbb{Z}^{2}$. We have to mod out by $\mathrm{H}_{2}\left(T^{2}\right)$ because each circle of Reeb orbits sweeps out a surface $\{\theta\} \times T^{2}$ in $S^{1} \times T^{2}$.

Lemma-Definition 10.10 Let $\alpha$ and $\alpha^{\prime}$ be Morse-Bott orbit sets corresponding to multiplicity functions $m$ and $m^{\prime}$. Then there is a canonical $\mathbb{Z}^{2}$-equivariant bijection between relative homology classes in $T^{3}$ and relative placements of periodic admissible paths,

$$
H_{2}\left(T^{3}, \alpha, \alpha^{\prime}\right)=\mathcal{R}\left(m, m^{\prime}\right)
$$

Proof Let $T$ and $T^{\prime}$ denote the supports of $m$ and $m^{\prime}$. For $Z \in H_{2}\left(T^{3}, \alpha, \alpha^{\prime}\right)$ and $\theta_{0} \in(\mathbb{R} / 2 \pi n \mathbb{Z}) \backslash\left(T \cup T^{\prime}\right)$, the intersection of $Z$ with the slice $\left\{\theta=\theta_{0}\right\} \subset T^{3}$ has a well-defined homology class as follows. If $Z$ is represented by a smooth surface $\Sigma$ intersecting $\left\{\theta=\theta_{0}\right\}$ transversely, then the intersection is a compact 1-manifold. We 
orient the intersection so that if $\{v, w\}$ is an oriented basis for the tangent space to $\Sigma$ at a point and $v$ is a positively oriented tangent vector to the intersection, then $w$ has positive $\partial_{\theta}$ component. Now define

$$
f\left(\theta_{0}\right):=\left[Z \cap\left\{\theta=\theta_{0}\right\}\right] \in H_{1}\left(T^{2}\right)=\mathbb{Z}^{2} .
$$

Then $f \in \mathcal{R}\left(m, m^{\prime}\right)$, and this defines the required bijection.

For an admissible almost complex structure $J$ as in Section 10.1 and $Z \in H_{2}\left(T^{3}, \alpha, \alpha^{\prime}\right)$, we can define $\mathcal{M}^{J}\left(\alpha, \alpha^{\prime}, Z\right)$ by analogy with Definition 10.5.

Definition 10.11 If $\Lambda$ and $\Lambda^{\prime}$ are periodic admissible paths of rotation number $n$ and period $\Gamma$ corresponding to the Morse-Bott orbit sets $\alpha$ and $\alpha^{\prime}$, let

$$
\mathcal{M}^{J}\left(\Lambda, \Lambda^{\prime}\right):=\mathcal{M}^{J}\left(\alpha, \alpha^{\prime}, \Lambda-\Lambda^{\prime}\right) .
$$

\subsection{Nesting of polygons and intersection positivity}

There is a simple but important constraint on $J$-holomorphic curves in $\mathbb{R} \times T^{3}$ in terms of the partial order $\leq$ from Section 2.3.

Proposition 10.12 Let $J$ be an admissible almost complex structure on $\mathbb{R} \times T^{3}$ for the contact form $\lambda_{n}$. Let $\Lambda$ and $\Lambda^{\prime}$ be periodic admissible paths of rotation number $n$ and period $\Gamma$. Then

$$
\mathcal{M}^{J}\left(\Lambda, \Lambda^{\prime}\right) \neq \varnothing \Longrightarrow \Lambda^{\prime} \leq \Lambda
$$

Proof Let $u \in \mathcal{M}^{J}\left(\Lambda, \Lambda^{\prime}\right)$. We want to show that for all $\theta \in \mathbb{R}$,

$$
\operatorname{det}\left(\begin{array}{c}
\cos \theta \\
\sin \theta
\end{array} \Lambda^{\prime}(\theta)-\Lambda(\theta)\right) \geq 0
$$

Choose $\theta_{0} \in(\mathbb{R} / 2 \pi n \mathbb{Z}) \backslash\left(T \cup T^{\prime}\right)$ such that $u$ is transverse to $\left\{\theta=\theta_{0}\right\} \subset \mathbb{R} \times T^{3}$. Consider a component of $u^{-1}\left\{\theta=\theta_{0}\right\}$, parametrized in an orientation-preserving manner by

$$
\rho=(s, x, y): S^{1} \longrightarrow\left\{\theta=\theta_{0}\right\} .
$$

Let $\tau$ denote the $S^{1}$ coordinate. By admissibility of $J$, the transversality assumption, and our orientation convention for the intersection, we have

$$
\sin \left(\theta_{0}\right) \frac{d x}{d \tau}-\cos \left(\theta_{0}\right) \frac{d y}{d \tau}>0
$$


(This can also be understood as positivity of intersections of $u$ with the leaves of the $J$-holomorphic foliation of $\left\{\theta=\theta_{0}\right\}$ by $\mathbb{R}$ times the Reeb flow.) Hence the homology class $\left(\rho_{x}, \rho_{y}\right) \in \mathbb{Z}^{2}$ of this component satisfies

$$
\operatorname{det}\left(\begin{array}{cc}
\rho_{x} & \cos \theta_{0} \\
\rho_{y} & \sin \theta_{0}
\end{array}\right)>0
$$

Adding this up for all components of $u^{-1}\left\{\theta=\theta_{0}\right\}$ proves equation (94) whenever $\theta \in \mathbb{R}$ is a lift of $\theta_{0} \in(R / 2 \pi n \mathbb{Z}) \backslash\left(T \cup T^{\prime}\right)$. By continuity, equation (94) holds for all $\theta \in \mathbb{R}$.

Remark 10.13 For a given $\theta_{0} \notin T \cup T^{\prime}$, the above argument shows that if equality holds in (94), then $u$ does not intersect the slice $\left\{\theta=\theta_{0}\right\}$.

\subsection{Spheres with two or three punctures, and degenerate and simple rounding}

Of particular interest is the "standard" almost complex structure $J_{\text {std }}$ on $\mathbb{R} \times T^{3}$ defined by

$$
\begin{aligned}
J_{\text {std }}\left(\partial_{s}\right) & :=\cos \theta \partial_{x}+\sin \theta \partial_{y}, \\
J_{\text {std }}\left(\partial_{\theta}\right) & :=-\sin \theta \partial_{x}+\cos \theta \partial_{y} .
\end{aligned}
$$

It is easy to check that $J_{\text {std }}$ is admissible. Since $J_{\text {std }}$ does not depend on $s, x$, or $y$, the action of $\mathbb{R} \times T^{2}$ on $\mathbb{R} \times T^{3}$ preserves $J_{\text {std }}$ and hence induces an action on $\mathcal{M}^{J_{\text {std }}}\left(\Lambda, \Lambda^{\prime}\right)$.

Definition 10.14 If $\Lambda, \Lambda^{\prime}$ are periodic admissible paths with rotation number $n$, let $\mathcal{M}_{0}^{J}\left(\Lambda, \Lambda^{\prime}\right)$ denote the set of irreducible, genus zero curves $u \in \mathcal{M}^{J}\left(\Lambda, \Lambda^{\prime}\right)$.

Proposition 10.15 Let $J$ be any $T^{2}$-invariant admissible almost complex structure on $\mathbb{R} \times T^{3}$ for the standard contact form $\lambda_{n}$. Suppose $\Lambda$ is a periodic admissible path of rotation number $n$, with two edges. Then:

(a) If $\Lambda^{\prime}$ is obtained from $\Lambda$ by degenerate rounding (see Section 9.1), then $\mathbb{R} \times T^{2}$ acts transitively on $\mathcal{M}_{0}^{J}\left(\Lambda, \Lambda^{\prime}\right)$ with $S^{1}$ stabilizer.

(b) If $\Lambda^{\prime}$ is obtained from $\Lambda$ by simple rounding, then $\mathbb{R} \times T^{2}$ acts freely and transitively on $\mathcal{M}_{0}^{J}\left(\Lambda, \Lambda^{\prime}\right)$.

Proof We will deduce the proposition from analogous results of Taubes [27], which hold for a similar contact form $\lambda_{T}$ on $S^{1} \times S^{2}$ and a $T^{2}$-invariant admissible almost 
complex structure $J_{T}$ on $\mathbb{R} \times S^{1} \times S^{2}$. For this purpose we will need to consider slightly more general contact forms on $T^{3}$. Namely, consider

$$
\lambda=a_{1}(\theta) d x+a_{2}(\theta) d y
$$

where

$$
a=\left(a_{1}, a_{2}\right): \mathbb{R} / 2 \pi n \mathbb{Z} \longrightarrow \mathbb{R}^{2} \backslash\{0\}
$$

has properties (i)-(iii) below. (In the following, if $v=\left(v_{1}, v_{2}\right)$ and $w=\left(w_{1}, w_{2}\right)$ are vectors in $\mathbb{R}^{2}$, then $v \times w:=v_{1} w_{2}-v_{2} w_{1}$.)

(i) The path $a$ has winding number $n$ around the origin in $\mathbb{R}^{2}$.

(ii) $a \times a^{\prime}>0$ for all $\theta$.

(iii) $a^{\prime} \times a^{\prime \prime}>0$ for all $\theta$.

Condition (ii) ensures that $\lambda$ is a contact form. The Reeb vector field is given by

$$
R=\frac{a_{2}^{\prime} \partial_{x}-a_{1}^{\prime} \partial_{y}}{a \times a^{\prime}}
$$

By (i) and (ii), $R$ has winding number $n$. Condition (iii) implies that $R$ turns to the left as $\theta$ increases. Hence we can reparametrize the $\theta$ coordinate (in exactly $n$ different ways) so that

(iv) $R$ is a positive multiple of $\cos \theta \partial_{x}+\sin \theta \partial_{y}$ for all $\theta$.

Of course the standard contact form $\lambda_{n}$ is recovered by taking $a=(\cos \theta, \sin \theta)$, which satisfies conditions (i)-(iv) above. Also, any two contact forms satisfying (i)-(iv) above are homotopic through such forms (by linear interpolation). For such a contact form, all of Section 10.2 and Section 10.3 holds verbatim (except for Remark 10.8). We will prove the proposition for any such contact form. We proceed in three steps.

Step 1 Denote the edges of $\Lambda$ by $\theta_{1}$ and $\theta_{2}$ with $\theta_{2}-\theta_{1} \in(0, \pi]$. Then the subset

$$
\left[\theta_{1}, \theta_{2}\right] \times T^{2} \subset T^{3}
$$

can be identified with a subset of $S^{1} \times S^{2}$ between two latitude lines, such that the pullback of Taubes's contact form $\lambda_{T}$ extends to a contact form $\lambda_{T}^{\prime}$ satisfying (i)-(iv) above. The pullback of Taubes's almost complex structure $J_{T}$ extends to a $T^{2}$-invariant admissible $J_{T}^{\prime}$ on $\mathbb{R} \times T^{3}$. By Remark 10.13, any $u \in \mathcal{M}^{J_{T}^{\prime}}\left(\Lambda, \Lambda^{\prime}\right)$ maps to $\mathbb{R} \times\left[\theta_{1}, \theta_{2}\right] \times T^{2}$, and an analogous argument works for $J_{T}$-holomorphic curves in $\mathbb{R} \times S^{1} \times S^{2}$. Hence Taubes's results are applicable to $\mathcal{M}_{T}^{J_{T}^{\prime}}\left(\Lambda, \Lambda^{\prime}\right)$. In particular, [27, Theorem A.1(c)] proves (a), and [27, Theorem A.2] proves (b), for $\lambda_{T}^{\prime}$ and $J_{T}^{\prime}$. 
Step 2 Now consider another contact form $\lambda$ on $T^{3}$ satisfying (i)-(iv) above, and an admissible $T^{2}$-invariant admissible $J$ on $\mathbb{R} \times T^{3}$. We can deform $\lambda_{T}^{\prime}$ to $\lambda$ through contact forms satisfying (i)-(iv), and for this family of contact forms we can find a family of $T^{2}$-invariant admissible almost complex structures interpolating between $J_{T}^{\prime}$ and $J$. The moduli spaces $\mathcal{M}_{0}\left(\Lambda, \Lambda^{\prime}\right)$ are smooth manifolds of the expected dimension throughout the deformation, as in [23, Theorem 1.2]. (For more general automatic transversality results see Wendl's doctoral thesis [29, Section 4.5.5].) By Gromov compactness (see the paper [4] by Bourgeois, Eliashberg, Hofer, Wysocki and Zehnder) the moduli spaces $\mathcal{M}_{0}\left(\Lambda, \Lambda^{\prime}\right) / \mathbb{R}$ are compact throughout the deformation, because by Propositions 2.10(b) and 10.12 and Lemma 10.7(b), there are never any broken pseudoholomorphic curves from $\Lambda$ to $\Lambda^{\prime}$. So the moduli spaces $\mathcal{M}_{0}\left(\Lambda, \Lambda^{\prime}\right) / \mathbb{R}$ for $J_{T}^{\prime}$ and for $J$ are diffeomorphic.

Step 3 Consideration of the Reeb orbits that appear at the ends of the $J$-holomorphic curves shows that in case (b), $\mathbb{R} \times T^{2}$ acts freely on $\mathcal{M}_{0}^{J}\left(\Lambda, \Lambda^{\prime}\right)$. This action must then be transitive, or else $\mathcal{M}_{0}^{J}\left(\Lambda, \Lambda^{\prime}\right)$ would be disconnected or not of the expected dimension, contradicting Step 2 . In case (a), $\mathbb{R} \times T^{2} / S^{1}$ acts freely on $\mathcal{M}_{0}^{J}\left(\Lambda, \Lambda^{\prime}\right)$, where $S^{1} \subset T^{2}$ is generated by the vector $\left(x_{\theta_{1}}, y_{\theta_{1}}\right)$. So $\mathbb{R} \times T^{2}$ must act transtively with $S^{1}$ stabilizer, or else again there would be a contradiction of Step 2.

The above proposition can also be deduced from work of Parker [20], which classifies genus zero pseudoholomorphic curves in $\mathbb{R} \times T^{3}$ for a degeneration of $J_{\text {std }}$, in terms of certain labeled graphs in $\mathbb{R}^{2} \backslash\{0\}$.

\subsection{The zero area constraint}

We now show that the sets of Reeb orbits that can appear at the ends of a $J_{\text {std }}-$ holomorphic curve in $\mathbb{R} \times T^{3}$ satisfy a codimension one constraint. This will be used in Section 11.3 to establish the No Double Rounding axiom for the embedded contact homology of $T^{3}$. To state the constraint, for $\theta \in \Theta_{n}$ we explicitly identify the circle of Reeb orbits in $\{\theta\} \times T^{2}$ with $S^{1}=\mathbb{R} / \mathbb{Z}$ via a map $\varphi$ defined as follows. If the Reeb orbit $\gamma$ contains a point $(\theta, x, y) \in T^{3}$, then we define

$$
\varphi(\gamma):=x_{\theta} y-y_{\theta} x+\frac{x_{\theta} y_{\theta}}{2} \in \mathbb{R} / \mathbb{Z} .
$$

If $u \in \mathcal{M}^{J}(\alpha, \beta)$, let $E_{+}(u)$ and $E_{-}(u)$ denote the set of positive and negative ends of $u$, respectively. For $e \in E_{ \pm}(u)$, let $\gamma(e)$ denote the corresponding embedded Reeb orbit and $m(e)$ the multiplicity of the end as defined in Section 10.1. 
Proposition 10.16 Let $u \in \mathcal{M}^{J_{\text {std }}}\left(\Lambda, \Lambda^{\prime}\right)$. Then

$$
\sum_{e \in E_{+}(u)} m(e) \varphi(\gamma(e))-\sum_{e \in E_{-}(u)} m(e) \varphi(\gamma(e))=0 \in \mathbb{R} / \mathbb{Z} .
$$

Proof (Conpare our earlier paper [10, Lemma A.2]) It follows from (95) that the 2-form $-d s d \theta+d x d y$ on $\mathbb{R} \times T^{3}$ annihilates any pair of tangent vectors of the form $\left(v, J_{\text {std }} v\right)$. Therefore

$$
\int_{C} u^{*}(d x d y)=\int_{C} u^{*}(d s d \theta)
$$

Now $\int_{C} u^{*}(d s d \theta)=0$ by Stokes' theorem, because the 1 -form $s d \theta$ vanishes along the Reeb orbits. Therefore $\int_{C} u^{*}(d x d y)=0$, ie the projection of $u$ to the $(x, y)$-torus has area zero. It follows from the identification (96) that this area is congruent modulo $\mathbb{Z}$ to the left side of equation (97).

\section{Embedded contact homology}

We now (in Section 11.2) outline the definition of the embedded contact homology of a contact 3-manifold. The idea is to count $J$-holomorphic curves with $I=1$, where $I$ is a certain upper bound on the index introduced in Section 11.1. In Section 11.3 we explain the correspondence between the embedded contact homology of $T^{3}$ and our combinatorial chain complexes. This will prove Theorem 1.2.

\subsection{The index inequality}

As in Section 10.1, let $Y$ be a closed oriented 3-manifold, let $\lambda$ be a contact 1-form on $Y$, and let $J$ be an admissible almost complex structure on $\mathbb{R} \times Y$.

If $\gamma$ is a Reeb orbit passing through a point $y \in Y$, then the linearization of the Reeb flow $R$ on the contact planes along $\gamma$ determines a linearized return map $P_{\gamma}: \xi_{y} \rightarrow \xi_{y}$. This is a symplectic linear map whose eigenvalues do not depend on $y$. The Reeb orbit $\gamma$ is nondegenerate if $P_{\gamma}$ does not have 1 as an eigenvalue. Assume now that all Reeb orbits, including multiply covered ones, are nondegenerate.

A Reeb orbit $\gamma$ is called elliptic or positive (resp. negative) hyperbolic when the eigenvalues of $P_{\gamma}$ are on the unit circle or the positive (resp. negative) real line respectively. If $\tau$ is a trivialization of $\xi$ over $\gamma$, one can then define the ConleyZehnder index $\mu_{\tau}(\gamma) \in \mathbb{Z}$. In our three-dimensional situation this is given explicitly as follows. For a positive integer $k$, let $\gamma^{k}$ denote the $k^{\text {th }}$ iterate of $\gamma$. If $\gamma$ is elliptic, 
then there is an irrational number $\phi \in \mathbb{R}$ such that $P_{\gamma}$ is conjugate in $\operatorname{SL}(2, \mathbb{R})$ to a rotation by angle $2 \pi \phi$, and

$$
\mu_{\tau}\left(\gamma^{k}\right)=2\lfloor k \phi\rfloor+1 .
$$

Here $2 \pi \phi$ is the total rotation angle with respect to $\tau$ of the linearized flow around the orbit. If $\gamma$ is positive (resp. negative) hyperbolic, then there is an even (resp. odd) integer $r$ such that the linearized flow around the orbit rotates the eigenspaces of $P_{\gamma}$ by angle $\pi r$ with respect to $\tau$, and

$$
\mu_{\tau}\left(\gamma^{k}\right)=k r .
$$

Let $\alpha=\left\{\left(\alpha_{i}, m_{i}\right)\right\}$ and $\beta=\left\{\left(\beta_{j}, n_{j}\right)\right\}$ be orbit sets as in Section 10.1. Suppose that $[\alpha]=[\beta]$ and let $Z \in H_{2}(Y, \alpha, \beta)$.

Definition 11.1 (Compare Eliashberg-Givental-Hofer [7]) If $u \in \mathcal{M}^{J}(\alpha, \beta ; Z)$, define the SFT index

$$
\begin{aligned}
& \operatorname{ind}(u):= \\
& \quad-\chi(C)+2 c_{1}\left(u^{*} \xi, \tau\right)+\sum_{e \in E_{+}(u)} \mu_{\tau}\left(\gamma(e)^{m(e)}\right)-\sum_{e \in E_{-}(u)} \mu_{\tau}\left(\gamma(e)^{m(e)}\right) .
\end{aligned}
$$

Here $\tau$ is a trivialization of the 2-plane bundle $\xi$ over the $\alpha_{i}$ 's and $\beta_{j}$ 's, and $c_{1}$ denotes the relative first Chern class with respect to $\tau$, see our earlier paper [9, Section 2].

The following proposition is the 3-dimensional case of a formula from [7] which is proved in the paper by Dragnev [6], using an index calculation by Schwarz [21].

Proposition 11.2 If $J$ is generic, and if $u \in \mathcal{M}^{J}(\alpha, \beta)$ has no multiply covered components, then $\mathcal{M}^{J}(\alpha, \beta)$ is a manifold near $u$ of dimension ind $(u)$.

Definition 11.3 (Hutchings [9]) Define the ECH index

$$
I(\alpha, \beta, Z):=c_{1}\left(\left.\xi\right|_{Z}, \tau\right)+Q_{\tau}(Z)+\sum_{i} \sum_{k=1}^{m_{i}} \mu_{\tau}\left(\alpha_{i}^{k}\right)-\sum_{j} \sum_{k=1}^{n_{j}} \mu_{\tau}\left(\beta_{j}^{k}\right) .
$$

Here $Q_{\tau}$ denotes the "relative intersection pairing", which is defined in [9, Section 2]. If $u \in \mathcal{M}^{J}(\alpha, \beta, Z)$, write $I(u):=I(\alpha, \beta, Z)$.

The following basic properties of $I$ are proved in [9]. First, $I(\alpha, \beta, Z)$ does not depend on the choice of $\tau$. Second, $I$ is additive in the sense that

$$
I(\alpha, \beta, Z)+I(\beta, \gamma, W)=I(\alpha, \gamma, Z+W) .
$$


Third, $I$ depends on $Z$ via the "index ambiguity formula"

$$
I(\alpha, \beta, Z)-I(\alpha, \beta, W)=\left\langle c_{1}(\xi)+2 \operatorname{PD}(\Gamma), Z-W\right\rangle .
$$

Fourth, the index mod 2 is given by

$$
I(\alpha, \beta, Z) \equiv \# h(\alpha)-\# h(\beta) \bmod 2
$$

where $\# h(\alpha)$ denotes the number of positive hyperbolic Reeb orbits in $\alpha$.

The key, nontrivial property of $I$ is the inequality (104) below which bounds the SFT index in terms of the ECH index.

Proposition 11.4 Suppose that $u \in \mathcal{M}^{J}(\alpha, \beta)$ does not multiply cover any component of its image and that the image of $u$ contains no trivial cylinders. Then

$$
\operatorname{ind}(u) \leq I(u)-2 \delta(u) \text {. }
$$

Moreover, if $T$ is a union of (possibly multiply covered) trivial cylinders, then

$$
I(u) \leq I(u \cup T)-2 \#(u \cap T) .
$$

Here $\delta(u)$ is a count of the singularities of $u$ with positive integer weights; in particular $\delta(u)=0$ iff $u$ is an embedding. Also, '\#' denotes the algebraic intersection number in $\mathbb{R} \times Y$. By intersection positivity (see McDuff [16]), $\#(u \cap T) \geq 0$, with equality iff $u \cap T=\varnothing$.

Proof Equation (104) follows from [9, Equation (18) and Proposition 6.1], and equation (105) holds as in [9, Proposition 7.1]. Note that these results in [9] are proved in a slightly different setting, where $Y$ is a mapping torus and a "local linearity" assumption is made. The asymptotic analysis needed to carry over these results to the present setting is done in Siefring's doctoral thesis [22].

The above proposition leads to strong restrictions on curves of low ECH index:

Corollary 11.5 Suppose $J$ is generic and $u \in \mathcal{M}^{J}(\alpha, \beta)$. Then:

(a) $I(u) \geq 0$.

(b) If $I(u)=0$, then the image of $u$ is a union of trivial cylinders.

(c) If $I(u)=1$, then $u$ contains one embedded component $u_{1}$ with $\operatorname{ind}\left(u_{1}\right)=$ $I\left(u_{1}\right)=1$. All other components of $u$ map to trivial cylinders that do not intersect $u_{1}$. 
Proof The image of $u$ consists of a union of $k$ irreducible non-multiply-covered $J$-holomorphic curves $u_{i}$, covered by $u$ with multiplicity $d_{i}$. Let $u^{\prime}$ be the union of $d_{i}$ different translates of $u_{i}$ in the $\mathbb{R}$ direction, for each $i$ such that $u_{i}$ is not a trivial cylinder. Let $T$ be the union of the components of $u$ that map to trivial cylinders. Note that if $u_{i}$ is a trivial cylinder then $\operatorname{ind}\left(u_{i}\right)=0$. So by equations (104) and (105),

$$
\sum_{i=1}^{k} d_{i} \operatorname{ind}\left(u_{i}\right)=\operatorname{ind}\left(u^{\prime}\right) \leq I\left(u^{\prime}\right)-2 \delta\left(u^{\prime}\right) \leq I(u)-2 \#\left(u^{\prime} \cap T\right)-2 \delta\left(u^{\prime}\right) .
$$

Since $J$ is generic, each nontrivial $u_{i}$ has $\operatorname{ind}\left(u_{i}\right)>0$ by Proposition 11.2 , since $\mathbb{R}$ acts nontrivially on the moduli space containing $u_{i}$. Also, $\delta\left(u^{\prime}\right)=0$ only if $u^{\prime}$ is embedded, which implies that all of the nontrivial $u_{i}$ 's are embedded. We can now read off the conclusions (a), (b), and (c) from the inequality (106).

\subsection{The definition of embedded contact homology}

Continue to assume that all Reeb orbits are nondegenerate.

\subsubsection{The chain complex}

Definition 11.6 An orbit set $\left\{\left(\alpha_{i}, m_{i}\right)\right\}$ is admissible if $m_{i}=1$ whenever $\alpha_{i}$ is hyperbolic.

Definition 11.7 If $\Gamma \in H_{1}(Y)$, then $C_{*}(Y, \lambda ; \Gamma)$ is the free $\mathbb{Z}$-module generated by admissible orbit sets $\alpha$ such that $[\alpha]=\Gamma$, and an ordering of the positive hyperbolic Reeb orbits in $\alpha$ is chosen. We declare that changing this ordering multiplies the generator by the sign of the reordering permutation.

Let $N$ denote the divisibility of the image of $c_{1}(\xi)+2 \operatorname{PD}(\Gamma)$ in $\operatorname{Hom}\left(H_{2}(Y), \mathbb{Z}\right)$. It follows from (103) and (102) that $I(\alpha, \beta, Z) \bmod N$ does not depend on $Z$ and defines a relative $\mathbb{Z} / N$ grading on $C_{*}(Y, \lambda ; \Gamma)$.

There is also a twisted chain complex defined for any subgroup $G \subset H_{2}(Y)$. Fix a "reference cycle", consisting of an oriented 1-dimensional submanifold $\rho \subset Y$ such that

$$
[\rho]=\Gamma \in H_{1}(Y)
$$

Definition 11.8 Let $\widetilde{C}_{*}(Y, \lambda ; \Gamma, G)$ be the free $\mathbb{Z}$-module generated by pairs $(\alpha,[W])$ where $\alpha$ is generator of $C_{*}(Y, \lambda ; \Gamma)$ and $[W] \in H_{2}(Y, \rho, \alpha) / G$. 
The $H_{2}(Y)$ action on $H_{2}(Y, \rho, \alpha)$ makes $\widetilde{C}_{*}(Y, \lambda ; \Gamma, G)$ into a free module over the group ring $\mathbb{Z}\left[H_{2}(Y) / G\right]$, with one generator for each admissible orbit set in the homology class $\Gamma$. If $G=H_{2}(Y)$, then $\widetilde{C}_{*}$ reduces to the "untwisted" complex in Definition 11.7. The "fully twisted" version has $G=\{0\}$.

11.2.2 The differential To define the differential, we first briefly review how to orient the relevant moduli spaces of $J$-holomorphic curves following Bourgeois-Mohnke [5]. For each Reeb orbit $\gamma$, there is a determinant line $\mathcal{O}_{\gamma}$ associated to $\bar{\partial}$ operators on the plane with asymptotics determined by the linearized Reeb flow along $\gamma$. (When $\gamma$ is an even multiple cover of a negative hyperbolic orbit, $\mathcal{O}_{\gamma}$ is only defined if one also chooses a marked point on the image of $\gamma$.) For each $\gamma$ we choose an orientation of $\mathcal{O}_{\gamma}$. When $\gamma$ is elliptic, there is a canonical "complex" orientation which we choose, cf Floer-Hofer [8, Theorem 2]. By [5], the above choices determine a sign for any transversely cut out ind $=1$ curve provided that the ends at positive hyperbolic orbits are ordered, and there are no ends at even covers of negative hyperbolic orbits.

In the following, assume that admissible orbit sets have orderings of the positive hyperbolic orbits chosen. If $J$ is generic, and if $\alpha$ and $\beta$ are homologous admissible orbit sets with $I(\alpha, \beta, Z)=1$, define a count

$$
\# \frac{\mathcal{M}^{J}(\alpha, \beta, Z)}{\mathbb{R}} \in \mathbb{Z}
$$

as follows. Declare two curves $u, u^{\prime} \in \mathcal{M}^{J}(\alpha, \beta, Z) / \mathbb{R}$ to be equivalent if their embedded components from Corollary $11.5(\mathrm{c})$ are the same up to translation, and if their other components cover each embedded trivial cylinder $\mathbb{R} \times \gamma$ with the same total multiplicity. In other words, $u$ and $u^{\prime}$ define the same current (modulo translation) in $\mathbb{R} \times Y$. The compactness argument of our earlier paper [9, Section 9.4] shows that there are only finitely many equivalence classes. For each equivalence class, if we discard the multiply covered trivial cylinders, then the resulting embedded curve has a sign by the previous paragraph. The count (108) is now the sum over the equivalence classes of the corresponding signs.

Definition 11.9 Define the differential

$$
\text { д: } \widetilde{C}_{*}(Y, \lambda ; \Gamma, G) \longrightarrow \widetilde{C}_{*-1}(Y, \lambda ; \Gamma, G)
$$

as follows. If $\alpha$ is an admissible orbit set with $[\alpha]=\Gamma$, then

$$
\partial(\alpha,[W]):=\sum_{I(\alpha, \beta, Z)=1} \# \frac{\mathcal{M}^{J}(\alpha, \beta, Z)}{\mathbb{R}} \cdot(\beta,[W+Z]) .
$$


Here the sum is over admissible orbit sets $\beta$ and relative homology classes $Z \in$ $H_{2}(Y, \alpha, \beta)$.

For technical reasons, we also need to consider, for a positive real number $L$, the subcomplex $\widetilde{C}_{*}^{<L}(Y, \lambda ; \Gamma, G)$ generated by orbit sets $\alpha$ with symplectic action $\mathcal{A}(\alpha)<$ $L$. By Lemma 10.7(a), the differential $\partial$ sends $\widetilde{C}_{*}^{<L}$ to itself.

A proof of the following is in preparation.

\section{Conjecture 11.10 (a) $\partial^{2}=0$.}

(b) The homology of $\widetilde{C}_{*}(Y, \lambda ; \Gamma, G)$, which we denote by $\widetilde{E C H}_{*}(Y, \lambda ; \Gamma, G)$, does not depend on $J$.

(c) The homology of $\widetilde{C}_{*}^{<L}(Y, \lambda ; \Gamma, G)$, which we denote by $\widetilde{E C H}_{*}^{<L}(Y, \lambda ; \Gamma, G)$, is invariant under deformations of $\lambda$ during which all orbits of action $<L$ are nondegenerate and no orbit has its action increase or decrease past $L$.

11.2.3 Morse-Bott version Suppose now that $\lambda$ has not only nondegenerate Reeb orbits but also $S^{1}$-families of Reeb orbits which are nondegenerate in the MorseBott sense. In principle one could define $\mathrm{ECH}$ in this situation along the lines of Bourgeois [2], without perturbing $\lambda$. However, the following definition is simpler to state. Each $S^{1}$-family of Reeb orbits, by a small perturbation of $\lambda$, can be replaced by two embedded Reeb orbits, one elliptic and one positive hyperbolic. Since there are typically infinitely many $S^{1}$-families of Reeb orbits, we cannot expect to perturb them all this way simultaneously. However, we can do this for all circles of Reeb orbits of symplectic action $<L$. So Conjecture 11.10 implies that $\widetilde{E C H}_{*}^{<L}(Y, \lambda ; \Gamma, G)$ is well-defined, and we then define ECH as the direct limit

$$
\widetilde{E C H}_{*}(Y, \lambda ; \Gamma, G):=\lim _{L \rightarrow \infty} \widetilde{E C H}_{*}^{<L}(Y, \lambda ; \Gamma, G) .
$$

In both the nondegenerate and Morse-Bott cases, we denote the "untwisted" ECH by

$$
E C H_{*}(Y, \lambda ; \Gamma):=\widetilde{E C H}_{*}\left(Y, \lambda ; \Gamma, H_{2}(Y)\right)
$$

\subsection{The example of $T^{3}$}

We now explain why the untwisted embedded contact homology of $T^{3}$, for the standard contact form $\lambda_{n}$ defined in (4) and (5), is computed by the combinatorial chain complex $\bar{C}_{*}(2 \pi n ; \Gamma)$. Also, the combinatorial chain complex $\widetilde{C}_{*}(2 \pi n ; \Gamma)$ computes a partially twisted version of the embedded contact homology of $T^{3}$. Below, $H_{*}\left(T^{2}\right)$ denotes the homology of an $x, y$ torus in $T^{3}$. 
Theorem 11.11 Assume Conjecture 11.10, so that ECH is well-defined. Then for $\Gamma \in$ $H_{1}\left(T^{2}\right)=\mathbb{Z}^{2}$, the embedded contact homology of $T^{3}$ is related to the combinatorial chain complexes by

$$
\begin{aligned}
\widetilde{E C H}_{*}\left(T^{3}, \lambda_{n} ; \Gamma, H_{2}\left(T^{2}\right)\right) & \simeq \widetilde{H}_{*}(2 \pi n ; \Gamma), \\
E C H_{*}\left(T^{3}, \lambda_{n} ; \Gamma\right) & \simeq \bar{H}_{*}(2 \pi n ; \Gamma) .
\end{aligned}
$$

Proof This is similar to the computation of the periodic Floer homology of a Dehn twist on a cylinder in our earlier paper [10], because the mapping torus flow for a negative Dehn twist on a cylinder is isomorphic to the Reeb flow on a subset of $T^{3}$ where $\theta$ ranges over an interval of length less than $\pi$. Thus we will carry over some lemmas from [10]. In making the translation, note that because the results in [10] are stated for positive Dehn twists, positive ends of $J$-holomorphic curves here correspond to negative (or "incoming"') ends there, and vice-versa. We now prove the theorem in five steps.

Step 1 We begin by defining an isomorphism of relatively graded $\mathbb{Z}\left[\mathbb{Z}^{2}\right]$-modules

$$
\widetilde{C}_{*}^{<L}\left(T^{3}, \lambda_{n} ; \Gamma, H_{2}\left(T^{2}\right)\right) \simeq \widetilde{C}_{*}^{<L}(2 \pi n ; \Gamma) .
$$

Here the right hand side denotes the subcomplex of $C_{*}(2 \pi n ; \Gamma)$ generated by admissible paths of length $<L$, as defined in (14).

Recall from Section 10.2 that for every $\theta \in \Theta_{n}$ there is an $S^{1}$ family of embedded Reeb orbits, such that each Reeb orbit $\gamma$ in the family has homology class

$$
[\gamma]=\left(0,\left(x_{\theta}, y_{\theta}\right)\right) \in H_{1}\left(T^{3}\right)=H_{1}\left(S^{1}\right) \oplus H_{1}\left(T^{2}\right) .
$$

After perturbation of $\lambda_{n}$, this family becomes an elliptic orbit $e_{\theta}$ and a positive hyperbolic orbit $h_{\theta}$, of approximately the same symplectic action.

To define (111), we first define an isomorphism of $\mathbb{Z}$-modules

$$
C_{*}^{<L}\left(T^{3}, \lambda_{n} ; \Gamma\right)=\bar{C}_{*}^{<L}(2 \pi n ; \Gamma) .
$$

Given a generator $\alpha$ of $C_{*}^{<L}\left(T^{3}, \lambda_{n} ; \Gamma\right)$, define a multiplicity function

$$
m: \mathbb{R} / 2 \pi n \mathbb{Z} \longrightarrow \mathbb{Z}_{\geq 0}
$$

by setting $m(\theta)$ equal to the total multiplicity of $e_{\theta}$ and $h_{\theta}$ in $\alpha$. By (93), this defines an admissible path $\Lambda$ of rotation number $n$ and period $\Gamma$, up to translation. By Remark 10.8 , the length of $\Lambda$ is less than $L$. If $\theta$ is an edge of $\Lambda$, label it ' $h$ ' if $h_{\theta}$ appears in $\alpha$, and label it ' $e$ ' otherwise. (By Definition $11.6, h_{\theta}$ cannot have multiplicity greater 
than 1 in $\alpha$.) For agreement with the SFT sign conventions of Eliashberg-GiventalHofer [7] and Bourgeois-Mohnke [5], we order the ' $h$ ' edges by the reverse of the ordering of the $h_{\theta}$ orbits in $\alpha$. This completes the definition of the isomorphism (112).

We next lift (112) to an isomorphism of $\mathbb{Z}\left[\mathbb{Z}^{2}\right]$-modules (111). (The possible lifts according to the prescription below will form an affine space over $\mathbb{Z}^{2}$.) To specify a lift, first choose a reference admissible path $\Lambda_{0}$ of rotation number $n$ and period $\Gamma$. Then choose the reference cycle $\rho$ as in (107) to be a union of Reeb orbits corresponding to $\Lambda_{0}$. By Lemma-Definition 10.10, this determines an isomorphism (111).

The left side of (111) has a well-defined relative $\mathbb{Z}$-grading, by (103). We claim that this agrees with the relative grading on the right hand side of (111) defined in equation (16). To see this, let $\alpha$ and $\beta$ be generators of $\widetilde{C}_{*}^{<L}(2 \pi n ; \Gamma)$. Denote the corresponding orbit sets by $\left\{\left(\alpha_{i}, m_{i}\right)\right\}$ and $\left\{\left(\beta_{j}, n_{j}\right)\right\}$. The correspondence of Lemma-Definition 10.10 then defines a relative homology class

$$
Z \in H_{2}\left(T^{3},\left\{\left(\alpha_{i}, m_{i}\right)\right\},\left\{\left(\beta_{j}, n_{j}\right)\right\}\right) / H_{2}\left(T^{2}\right) .
$$

We need to show that with this $Z$, the right hand sides of (101) and (16) agree. Observe that the contact 2-plane field $\xi$ has a nonvanishing section $\partial_{\theta}$ over $T^{3}$, and this gives rise to a global trivialization $\tau$ of $\xi$. We then have

$$
c_{1}\left(\left.\xi\right|_{Z}, \tau\right)=0
$$

Also, if $\alpha$ or $\beta$ contains $e_{\theta}$ or $h_{\theta}$ with multiplicity $k$, then for a sufficiently small perturbation of the Morse-Bott contact form, $0<\phi<1 / k$ in equation (98) and $r=0$ in equation (99), so

$$
\mu_{\tau}\left(e_{\theta}^{k}\right)=1, \quad \mu_{\tau}\left(h_{\theta}^{k}\right)=0
$$

Therefore

$$
\sum_{i} \sum_{k=1}^{m_{i}} \mu_{\tau}\left(\alpha_{i}^{k}\right)-\sum_{j} \sum_{k=1}^{n_{j}} \mu_{\tau}\left(\beta_{j}^{k}\right)=(\ell(\alpha)-\# h(\alpha))-(\ell(\beta)-\# h(\beta)) .
$$

By equations (113) and (115), to complete the proof that the relative indices agree, we must show that

$$
Q_{\tau}(Z)=2 \int_{P} x d y
$$

This follows as in our earlier paper [9, Lemma 3.7] when the admissible path underlying $\beta$ is obtained from that of $\alpha$ by nondegenerate rounding. The case of degenerate rounding follows by an easy generalization of this. By induction using Proposition 2.13, equation (116) holds for any two generators $\alpha$ and $\beta$. 
Step 2 Choose a small perturbation of $\lambda_{n}$, a generic almost complex structure $J$, and orientations of the determinant lines $\mathcal{O}_{h_{\theta}}$ needed to define the ECH differential $\partial$ on the left hand side of (111). We assume that the perturbed contact form agrees with $\lambda_{n}$ away from an $\varepsilon$-neighborhood of the circles of Reeb orbits of $\lambda_{n}$ with action $<L$, where $\varepsilon$ is small with respect to $L$. We claim that the differential $\partial$, regarded as a differential on the right hand side of (111), satisfies the Nesting, Connectedness, Label Matching, and Locality axioms of Section 9.1 where applicable, ie whenever $\alpha$ and $\beta$ have length $<L$.

If $\alpha$ and $\beta$ are generators of the right hand side of (111), let $\mathcal{M}^{J}(\alpha, \beta)$ denote the $J$-holomorphic curves counted by the differential coefficient $\langle\partial \alpha, \beta\rangle$. To prove the Nesting axiom, suppose there exists $C \in \mathcal{M}^{J}(\alpha, \beta)$ where $\alpha$ and $\beta$ have length $<L$ and $\beta \not \leq \alpha$. Then there exists $\theta_{0} \in \mathbb{R} / 2 \pi n \mathbb{Z}$ such that

$$
\operatorname{det}\left(\begin{array}{cc}
\cos \theta_{0} \\
\sin \theta_{0}
\end{array} \beta\left(\theta_{0}\right)-\alpha\left(\theta_{0}\right)\right)<0 .
$$

By continuity we can choose $\theta_{0}$ such that $\tan \theta_{0}$ is not a rational number of denominator $\leq L$. We can assume that $\varepsilon$ above is sufficiently small that the perturbed contact form agrees with $\lambda_{n}$ when $\theta=\theta_{0}$. We then get a contradiction as in Proposition 10.12.

The Connectedness axiom holds because if $D(\alpha, \beta)$ is disconnected, then as in Remark 10.13 , if $\varepsilon$ is sufficiently small, then a $J$-holomorphic curve in $\mathcal{M}^{J}(\alpha, \beta)$ has at least two non-trivial components. By Corollary 11.5, such a curve cannot exist unless $I(\alpha, \beta) \geq 2$, whence $\langle\partial \alpha, \beta\rangle=0$.

Before continuing, we need some restrictions on the topological complexity of the $J$-holomorphic curves counted by $\partial$. Suppose that $u \in \mathcal{M}^{J}(\alpha, \beta)$ and $I(\alpha, \beta)=1$. By Corollary 11.5, $u$ has one component $u_{1}$ which does not map to a trivial cylinder, with $\operatorname{ind}\left(u_{1}\right)=1$. Let $g\left(u_{1}\right)$ denote the genus of the domain of $u_{1}$, let $e_{+}\left(u_{1}\right)$ denote the number of positive ends of $u_{1}$ at elliptic Reeb orbits, and let $h\left(u_{1}\right)$ denote the number of positive or negative ends of $u_{1}$ at hyperbolic Reeb orbits. Since ind $\left(u_{1}\right)=1$, it follows from equations (100), (113), and (114) that

$$
2 g\left(u_{1}\right)+2 e_{+}\left(u_{1}\right)+h\left(u_{1}\right)=3 .
$$

We claim now that for each $J$-holomorphic curve counted by $\partial$, the nontrivial component $u_{1}$ has genus zero. By equation (117) the only other possibility is that $g\left(u_{1}\right)=1$; $u_{1}$ has one positive end, which is hyperbolic; and all negative ends of $u_{1}$ are elliptic. By Nesting, $u_{1}$ has only one negative end, which corresponds to the same edge as its positive end; then $u_{1} \in \mathcal{M}^{J}(\alpha, \beta)$ with $I(\alpha, \beta)=-1$, contradicting Corollary 11.5(a).

We now prove the Label Matching axiom. To prove the first sentence of the axiom, if $\langle\partial \alpha, \beta\rangle \neq 0$ and if two edges of $\alpha$ and $\beta$ at angle $\theta_{0}$ agree but have different labels, 
then as in Remark 10.13, the contributing $J$-holomorphic curves include nontrivial components living in an $\varepsilon$-neighborhood of the slice $\left\{\theta=\theta_{0}\right\}$. By equation (117), the only such nontrivial curves that can arise are cylinders with a positive end at $e_{\theta_{0}}$ and a negative end at $h_{\theta_{0}}$. By Morse-Bott theory, cf Bourgeois [2], these count with opposite signs as in the Morse homology of $S^{1}$. The second sentence of the Label Matching axiom holds because if $\alpha$ and $\beta$ fail this condition, then the nontrivial component of any $u \in \mathcal{M}(\alpha, \beta)$ would have a negative hyperbolic end, a positive elliptic end, and at least one other positive end, violating equation (117).

To prove the Locality axiom, let $\alpha^{\prime}$ and $\beta^{\prime}$ be defined as in the statement of the axiom. By (89) and the analogue of [10, Lemma 3.9], taking the union with trivial cylinders defines a map $\mathcal{M}\left(\alpha^{\prime}, \beta^{\prime}\right) / \mathbb{R} \rightarrow \mathcal{M}(\alpha, \beta) / \mathbb{R}$, which is a bijection on the equivalence classes of curves that the differential counts. Our ordering convention in Step 1 ensures that this bijection is orientation-preserving, so $\langle\partial \alpha, \beta\rangle=\left\langle\partial \alpha^{\prime}, \beta^{\prime}\right\rangle$.

Step 3 The proof of Lemma 9.2 then shows that we can write $\partial=\partial_{0}+\partial_{1}$, where $\left\langle\partial_{0} \alpha, \beta\right\rangle \neq 0$ only if $\langle\delta \alpha, \beta\rangle \neq 0$, and $\left\langle\partial_{1} \alpha, \beta\right\rangle \neq 0$ only if $\beta$ is obtained from $\alpha$ by "double rounding", ie rounding two adjacent corners and losing three ' $h$ 's. Also $\partial^{2}=0$ implies that $\partial_{0}^{2}=0$, because $\widetilde{C}_{*}^{<L}(2 \pi n ; \Gamma)$ is filtered by $I-\# h$, and $\partial_{0}$ is the differential on the associated graded complex.

We henceforth orient all of the $\mathcal{O}_{h_{\theta}}$ 's as follows. As mentioned above there are two $J-$ holomorphic cylinders from $e_{\theta}$ to $h_{\theta}$ which count with opposite signs. The projections of these cylinders to $T^{2}$ have areas of opposite sign. (The areas differ by 1.) We choose the orientation of $\mathcal{O}_{h_{\theta}}$ so that the cylinder whose projection to $T^{2}$ has positive area counts with positive sign.

With the above orientation choices, as in [10, Lemma 3.15(b)], $\partial_{0}$ does not depend on the small perturbation of the contact form, $J$, or $L$. In conclusion, $\partial_{0}$ is a well-defined differential on all of $\widetilde{C}_{*}(2 \pi n)$ satisfying the Nesting, Connectedness, Label Matching, Locality, and No Double Rounding axioms.

Step 4 We claim now that, possibly after changing some signs in the isomorphism (111), the differential $\partial_{0}$ also satisfies the Degenerate Rounding and Simple Rounding axioms. To prove either of these axioms, by Locality we may assume that $\alpha$ has only two edges. By the invariance of $\partial_{0}$, we may assume that $J$ is close to the almost complex structure $J_{\text {std }}$ defined in equation (95). Up to signs, the Degenerate Rounding and Simple Rounding axioms now follow from Proposition 10.15 by using Morse-Bott theory as in [10, Section 3.8]. 
To understand the signs, recall from Lemma 6.20 that $\widetilde{S L}_{2} \mathbb{Z}$ acts on $\bar{C}_{*}(2 \pi n)$. In fact, an element $(A, f) \in \widetilde{S L}_{2} \mathbb{Z}$ gives rise to a diffeomorphism of $T^{3}$ sending

$$
(\theta,(x, y)) \longmapsto(f(\theta), A(x, y))
$$

and preserving the Reeb direction, and this induces the action on the generators of $\bar{C}_{*}(2 \pi n)$. As in [10, Lemma 3.16], with the orientation choices of Step 3, the cofficients of $\partial_{0}$ are $\widetilde{S L}_{2} \mathbb{Z}$-invariant. Thus there are only two degenerate rounding coefficients (depending on whether the ' $h$ ' edge comes before or after the rounded corner) and three simple rounding coefficients, each of which is +1 or -1 .

We claim that the two degenerate rounding coefficients have opposite signs, and the three simple rounding coefficients are related schematically by

$$
\left\langle\partial_{0}(e h), e\right\rangle=-\left\langle\partial_{0}(h e), e\right\rangle=\left\langle\partial_{0}(h h), h\right\rangle .
$$

A shortcut to checking these signs is to consider the fully twisted chain complex with its differential $\widetilde{\partial}$, cf Section 12.1.1. Our previous discussion of the cylinders from $e_{\theta}$ to $h_{\theta}$ implies that if $\Lambda$ has one edge then

$$
\widetilde{\partial} E_{\Lambda}=t^{k}(1-t) H_{\Lambda}
$$

where $t$ is a group ring generator corresponding to $H_{2}\left(T^{2}\right)$ and $k$ is some integer depending on $\Lambda$. (One can arrange that $k=0$, but this is not necessary here.) Each degenerate rounding coefficient is now plus or minus a power of $t$. Then applying $\widetilde{\partial}^{2}=0$ to a generator $E_{\Lambda}$ where $\Lambda$ has two edges and a corner of angle $\pi$ implies that the two degenerate rounding coefficients have opposite sign. Next, applying $\partial^{2}=0$ to simple triangles with two edges labeled ' $h$ ' establishes the relations (118) between the three simple rounding coefficients.

Thus the Degenerate Rounding and Simple Rounding axioms hold up to a global sign in each. To make both of these signs positive, consider the automorphism $\phi_{h}$ of $\widetilde{C}_{*}(2 \pi n)$ that sends $\alpha \mapsto(-1)^{\# h(\alpha)} \alpha$, and similarly let $\phi_{e}$ be the automorphism of $\widetilde{C}_{*}(2 \pi n)$ that multiplies a generator $\alpha$ by $(-1)$ to the number of elliptic orbits in $\alpha$. Then composing the isomorphism (111) with $\phi_{e}$ will change the degenerate rounding sign but not the simple rounding sign, while composing the isomorphism (111) with $\phi_{h}$ will change both signs.

Step 5 We now complete the proof of the theorem. By the previous steps, the chain complex $\left(\widetilde{C}_{*}(2 \pi n), \partial_{0}\right)$ satisfies all the axioms of Section 9.1. By Proposition 9.1, $\partial_{0}=\delta$. It follows from Proposition 10.16 as in [10, Lemma A.1(a)] that for any $L$, the perturbation of $\lambda_{n}$ and $J_{\text {std }}$ can be chosen so that $\partial_{1}=0$. Hence

$$
\widetilde{H}_{*}^{<L}\left(T^{3}, \lambda_{n} ; \Gamma, H_{2}\left(T^{2}\right)\right)=\widetilde{H}_{*}^{<L}(2 \pi n ; \Gamma) .
$$


Taking the direct limit as $L \rightarrow \infty$ proves the isomorphism (109). The isomorphism (110) follows because all of the chain maps in the proof of (109) are $\mathbb{Z}^{2}$-equivariant.

\section{Concluding remarks}

\subsection{Additional structure on ECH}

We now briefly describe some additional structures on ECH in general and their combinatorial manifestations in the example of $T^{3}$, assuming Conjecture 11.10.

12.1.1 The fully twisted ECH of $T^{3}$ Similarly to Theorem 11.11, the fully twisted embedded contact homology of $T^{3}$ is described combinatorially by

$$
\widetilde{E C H}_{*}\left(T^{3}, \lambda_{n} ; \Gamma, 0\right) \simeq H_{*}\left(\widetilde{C}_{*}(2 \pi n ; \Gamma) \otimes \mathbb{Z}\left[t, t^{-1}\right], \widetilde{\delta}\right)
$$

where $\widetilde{\delta}$ is defined below. First define a map

$$
\delta^{\prime}: \widetilde{C}_{*}(2 \pi n ; \Gamma) \longrightarrow \widetilde{C}_{*-1}(2 \pi n ; \Gamma)
$$

as follows. If $\alpha$ is a generator of $\widetilde{C}_{*}(2 \pi n ; \Gamma)$, define $\delta^{\prime}(\alpha)$ to be the sum of all ways of relabeling an ' $e$ ' edge of $\alpha$ by ' $h$ ' and making it last in the ordering. For example, $\delta^{\prime}\left(E_{\Lambda}\right)=H_{\Lambda}$. Note that $\delta^{\prime}$ is essentially a special case of the operator $K_{\theta_{1}, \theta_{2}}$ defined in Section 4, with $\theta_{2}=\theta_{1}+2 \pi n$. So as in Proposition 4.5,

$$
\delta^{\prime} \delta+\delta \delta^{\prime}=0 .
$$

We now define

$$
\widetilde{\delta}:=\delta+(1-t) \delta^{\prime}
$$

It is easy to see that $\left(\delta^{\prime}\right)^{2}=0$. Together with $\delta^{2}=0$ and equation (120), this implies that $\widetilde{\delta}^{2}=0$.

In the correspondence (119), $t$ is an extra group ring generator corresponding to a generator of $H_{2}\left(T^{2}\right)$. The $(1-t) \delta^{\prime}$ term in the differential arises from the twisted Morse complex of the circles of Reeb orbits of $\lambda_{n}$.

Similarly to Theorem 7.1,

$$
H_{*}\left(\widetilde{C}_{*}(2 \pi n ; \Gamma) \otimes \mathbb{Z}\left[t, t^{-1}\right], \widetilde{\delta}\right) \simeq\left\{\begin{array}{cl}
\mathcal{I}\left(\mathbb{Z}^{3}\right), & \Gamma=0, *=0, \\
\mathbb{Z}, & \Gamma=0, *=1,3, \ldots, \\
0, & \text { otherwise. }
\end{array}\right.
$$

Here $\mathcal{I}\left(\mathbb{Z}^{3}\right)$ denotes the augmentation ideal in $\mathbb{Z}\left[\mathbb{Z}^{3}\right]$, and $\mathbb{Z}$ denotes the $\mathbb{Z}\left[\mathbb{Z}^{3}\right]$-module with one generator on which $\mathbb{Z}^{3}$ acts by the identity. 
12.1.2 The contact element In general there is a canonical homology class

$$
c(\lambda) \in \widetilde{E C H}_{0}(Y, \lambda ; 0, G) .
$$

This is the homology class of the chain complex generator $\alpha=\varnothing$, namely the empty set of Reeb orbits. (This is well-defined in the twisted cases if we choose the reference cycle $\rho=0$ in (107).) Note that $\partial \alpha=0$, because by convexity any $J$-holomorphic curve in $\mathbb{R} \times Y$ has at least one positive end. We conjecture that the homology class (123) depends only on the contact structure $\xi$.

In the untwisted ECH of $T^{3}$, the class $c\left(\lambda_{n}\right)$ corresponds to the homology class of a 0 -gon in the combinatorial homology $\bar{H}_{0}(2 \pi n ; 0)$. This homology class is a generator if $n=1$, and 0 if $n>1$. On the other hand, in the fully twisted ECH of $T^{3}$, the isomorphism (122) can be chosen so that

$$
c\left(\lambda_{n}\right)=(1-t)^{n} \in \mathcal{I}\left(\mathbb{Z}^{3}\right) .
$$

\subsubsection{The action of $H_{1}$ An element}

$$
\zeta \in H_{1}(Y) / \text { Tors }=\operatorname{Hom}\left(H^{1}(Y ; \mathbb{Z}), \mathbb{Z}\right)=\operatorname{Hom}\left(H_{2}(Y), \mathbb{Z}\right)
$$

induces a degree -1 map

$$
\partial_{\zeta}: \widetilde{E C H}_{*}(Y, \lambda ; \Gamma, G) \longrightarrow \widetilde{E C H}_{*-1}(Y, \lambda ; \Gamma, G) .
$$

The map $\partial_{\zeta}$ is defined by an algebraic operation on the fully twisted chain complex, by analogy with a construction due to Ozsváth and Szabó [18]. We will also give an equivalent geometric definition in Section 12.1.4.

In general, suppose we are given a free chain complex $\left(\widetilde{C}_{*}, \partial\right)$ over a group ring $\mathbb{Z}[H]$ and a homomorphism $\zeta: H \rightarrow \mathbb{Z}$. Then $\zeta$ induces a $\mathbb{Z}$-linear map $\widetilde{\zeta}: \mathbb{Z}[H] \rightarrow \mathbb{Z}[H]$ sending $\sum_{h} a_{h} h \mapsto \sum_{h} \zeta(h) a_{h} h$ and satisfying

$$
\widetilde{\zeta}(x y)=\widetilde{\zeta}(x) y+x \widetilde{\zeta}(y) .
$$

Choose a basis $\left\{x_{i} \mid i \in I\right\}$ for $\widetilde{C}_{*}$ over $\mathbb{Z}[H]$, and define a $\mathbb{Z}[H]$-linear map $\partial_{\zeta}: \widetilde{C}_{*} \longrightarrow$ $\widetilde{C}_{*-1}$ by

$$
\partial_{\zeta}\left(x_{i}\right):=\sum_{j \in I} \widetilde{\zeta}\left(\left\langle\partial x_{i}, x_{j}\right\rangle\right) x_{j}
$$

Then $\partial^{2}=0$ and equation (125) imply that $\partial \circ \partial_{\zeta}+\partial_{\zeta} \circ \partial=0$. Equation (125) further implies that the map that $\partial_{\zeta}$ induces on homology is natural and hence does not depend on the choice of basis. Also $\partial_{\zeta_{1}+\zeta_{2}}=\partial_{\zeta_{1}}+\partial_{\zeta_{2}}$, and $2 \partial_{\zeta} \circ \partial_{\zeta}=0$ on homology. 
Specializing this to the fully twisted $\mathrm{ECH}$, if $\zeta \in \operatorname{Hom}\left(H_{2}(Y), \mathbb{Z}\right)$, then we obtain a chain map

$$
\partial_{\zeta}: \widetilde{C}_{*}(Y, \lambda ; \Gamma, 0) \longrightarrow \widetilde{C}_{*-1}(Y, \lambda ; \Gamma, 0) .
$$

Modding out by $G$ and passing to homology gives the map (124).

We now consider the example of $T^{3}$ and compute the map

$$
\partial_{\zeta}: E C H_{*}\left(T^{3}, \lambda_{n} ; 0\right) \longrightarrow E C H_{*-1}\left(T^{3}, \lambda_{n} ; 0\right)
$$

in terms of the generators in Proposition 8.3. Recall that we have been using a basis $\{t, x, y\}$ for $H_{2}\left(T^{3}\right)$; we denote the dual basis of $H_{1}\left(T^{3}\right)$ by the same letters $\{t, x, y\}$. It then follows from (121) that $\partial_{t}$ is induced by $-\delta^{\prime}$. Observe that $\delta^{\prime}$ commutes with the splicing chain map $S$ defined in Section 7.1. It follows by induction on $n$ that in Proposition 8.3 one can take $q_{k-1}:=\delta^{\prime} p_{k}$ for $k>0$. By equation (120), one can then take $u_{k}:=\delta^{\prime} s_{k}$ and $v_{k}:=\delta^{\prime} t_{k}$, whence

$$
\partial_{t}\left(s_{k}\right)=-u_{k}, \quad \partial_{t}\left(t_{k}\right)=-v_{k}
$$

for $k>0$. It is not hard to obtain (126) for $k=0$ as well. From the bigrading and $\left(\delta^{\prime}\right)^{2}=0$, we find that $\partial_{t}$ of all other generators is zero. We also read off from Proposition 8.3 that

$$
\begin{gathered}
\partial_{x}\left(s_{k}\right)=p_{k}, \quad \partial_{x}\left(w_{k}\right)=-v_{k}, \\
\partial_{y}\left(t_{k}\right)=p_{k}, \quad \partial_{y}\left(w_{k}\right)=u_{k},
\end{gathered}
$$

and $\partial_{x}$ and $\partial_{y}$ of all other generators is zero.

12.1.4 The homology operation $U$ We now describe a degree -2 operation on the embedded contact homology

$$
U: \widetilde{E C H}_{*}(Y, \lambda ; \Gamma, G) \longrightarrow \widetilde{E C H}_{*-2}(Y, \lambda ; \Gamma, G) .
$$

Fix a point $z \in Y$ which is not on any Reeb orbit. Let $\mathcal{M}^{J}(\alpha, \beta, Z)^{z}$ denote the set of curves $u \in \mathcal{M}^{J}(\alpha, \beta, Z)$ with a marked point mapping to $(0, z) \in \mathbb{R} \times Y$. For a suitable orientation on $\mathcal{M}^{J}(\alpha, \beta, Z)^{z}$, define

$$
\begin{aligned}
U_{z}: \widetilde{C}_{*}^{<L}(Y, \lambda ; \Gamma, G) & \longrightarrow \widetilde{C}_{*-2}^{<L}(Y, \lambda ; \Gamma, G), \\
(\alpha,[W]) & \longmapsto \sum_{I(\alpha, \beta, Z)=2} \# \mathcal{M}^{J}(\alpha, \beta, Z)^{z} \cdot(\beta,[W+Z]) .
\end{aligned}
$$

We expect to prove similarly to Conjecture 11.10 that $U_{z}$ is a chain map, and a generic path $P$ from $z$ to $z^{\prime}$ induces a chain homotopy $K_{P}$ between $U_{z}$ and $U_{z^{\prime}}$. The chain homotopy counts $J$-holomorphic curves with $I=1$ that contain a marked point mapping to $\{0\} \times P \subset \mathbb{R} \times Y$. Then $U_{z}$ induces a well defined map $U$ on $\mathrm{ECH}$. 
We remark that if $P$ is a loop, then $K_{P}$ is equivalent to the map $\partial_{[P]}$ defined in Section 12.1.3.

For $Y=T^{3}$, if we take $z=(\theta, x, y)$, then the geometric chain map $U_{z}$ defined above is related to the combinatorial chain map $U_{\theta}$ defined in Section 4 as follows. Similarly to Theorem 11.11, under the isomorphism (111) we have

$$
U_{z}=U_{\theta}+U^{\prime}
$$

where $\left\langle U^{\prime} \alpha, \beta\right\rangle \neq 0$ only if $\beta$ is obtained from $\alpha$ by rounding two consecutive corners and losing two ' $h$ 's, or rounding three consecutive corners and losing four ' $h$ 's. Without knowing anything more about the "error term" $U^{\prime}$, we can show that $U_{z}$ and $U_{\theta}$ induce the same map on $E C H_{*}\left(T^{3}, \lambda_{n} ; 0\right)$. That is, the generators in Proposition 8.3 can be chosen so that equations (67) and (68) hold in homology with $U=U_{\theta}$ replaced by $U_{z}$. We obtain the equations (67), ie $U^{\prime} p_{k+1}=U^{\prime} s_{k+1}=U^{\prime} t_{k+1}=0$, just by counting the number of ' $h$ 's in the generators. We then obtain the equations (68) by noting that $U_{z}$ commutes with the map $\partial_{t}$ defined in Section 12.1.3, and using equation (126).

\subsection{Some other 3-manifolds}

12.2.1 $S^{1} \times S^{2} \quad$ The methods of this paper can be modified to compute the ECH of $S^{1} \times S^{2}$ with the contact form $\lambda_{T}$ studied by Taubes in [27]. Apparently

$$
\widetilde{E C H}_{*}\left(S^{1} \times S^{2}, \lambda_{T} ; \Gamma, 0\right) \simeq \begin{cases}\mathbb{Z}, & \Gamma=\left[S^{1}\right] \times[p t], *=i_{0}, i_{0}+2, \ldots, \\ 0, & \text { otherwise }\end{cases}
$$

Here $i_{0}$ is a certain odd value of the grading. A generator in degree $i_{0}$ is given (after perturbation from the Morse-Bott setting) by a hyperbolic orbit in $S^{1}$ cross the equator of $S^{2}$. This calculation is relevant to Taubes's program [24; 26], provides more evidence for Conjecture 1.1, and shows that ECH need not vanish for an overtwisted contact form.

12.2.2 Torus bundles Let $Y$ be the $T^{2}$-bundle over $S^{1}$ with monodromy $A^{-1} \in$ $S L_{2} \mathbb{Z}$. Choose a lift $(A, f) \in \widetilde{S L_{2} \mathbb{Z}}$ of $A$, as in Section 6.3, such that $f(\theta)>\theta$ for all $\theta$, and $f$ has rotation number in $(2 \pi(n-1), 2 \pi n]$ with $n>0$. Also choose a lift $\left(\left(A^{-1}\right)^{T}, g\right)$ of the inverse transpose $\left(A^{-1}\right)^{T}$ corresponding to the same $n$. Then the diffeomorphism

$$
\begin{aligned}
\mathbb{R} \times T^{2} & \longrightarrow \mathbb{R} \times T^{2}, \\
(\theta,(x, y)) & \longmapsto(g(\theta), A(x, y))
\end{aligned}
$$

preserves the contact structure given by the kernel of the standard contact form (5), and thus defines a contact structure on the quotient, which is diffeomorphic to $Y$. This 
contact structure is the kernel of a $\theta$-dependent rescaling of the contact form (5). The rescaling can be chosen so that the Reeb vector field rotates to the left as $\theta$ increases, cf Section 10.4. Similarly to Theorem 11.11, the ECH of $Y$ for such a contact form and for

$$
\Gamma \in \mathbb{Z}^{2} / \operatorname{Im}(1-A) \subset H_{1}(Y)
$$

is computed by a "twisted" variant of the combinatorial complex $\bar{C}_{*}(2 \pi n ; \Gamma)$. In this chain complex, which we denote by $\bar{C}_{*}(A, n ; \Gamma)$, the periodicity condition in Definition 2.2 is replaced by the conditions

$$
\begin{gathered}
\frac{d \Lambda}{d \theta} \circ f=A \circ \frac{d \Lambda}{d \theta}, \\
{[\Lambda(f(\theta))-\Lambda(\theta)]=\Gamma .}
\end{gathered}
$$

It is an interesting problem to compute the homology of this complex.

\section{References}

[1] C Abbas, K Cieliebak, H Hofer, The Weinstein conjecture for planar contact structures in dimension three, Comment. Math. Helv. 80 (2005) 771-793 MR2182700

[2] F Bourgeois, A Morse-Bott approach to contact homology, from: "Symplectic and contact topology: interactions and perspectives (Toronto, ON/Montreal, QC, 2001)", Fields Inst. Commun. 35, Amer. Math. Soc., Providence, RI (2003) 55-77 MR1969267

[3] F Bourgeois, V Colin, Homologie de contact des variétés toroïdales, Geom. Topol. 9 (2005) 299-313 MR2116317

[4] F Bourgeois, Y Eliashberg, H Hofer, K Wysocki, E Zehnder, Compactness results in symplectic field theory, Geom. Topol. 7 (2003) 799-888 MR2026549

[5] F Bourgeois, K Mohnke, Coherent orientations in symplectic field theory, Math. Z. 248 (2004) 123-146 MR2092725

[6] D L Dragnev, Fredholm theory and transversality for noncompact pseudoholomorphic maps in symplectizations, Comm. Pure Appl. Math. 57 (2004) 726-763 MR2038115

[7] Y Eliashberg, A Givental, H Hofer, Introduction to symplectic field theory, Geom. Funct. Anal. (2000) 560-673 MR1826267

[8] A Floer, H Hofer, Coherent orientations for periodic orbit problems in symplectic geometry, Math. Z. 212 (1993) 13-38 MR1200162

[9] M Hutchings, An index inequality for embedded pseudoholomorphic curves in symplectizations, J. Eur. Math. Soc. (JEMS) 4 (2002) 313-361 MR1941088

[10] M Hutchings, M Sullivan, The periodic Floer homology of a Dehn twist, Algebr. Geom. Topol. 5 (2005) 301-354 MR2135555 
[11] M Hutchings, M Thaddeus, Periodic Floer homology, (in preparation)

[12] P Kronheimer, T Mrowka, Floer homology for Seiberg-Witten monopoles, (in preparation)

[13] P B Kronheimer, T S Mrowka, Monopoles and contact structures, Invent. Math. 130 (1997) 209-255 MR1474156

[14] P Kronheimer, T Mrowka, P Ozsváth, Z Szabó, Monopoles and lens space surgeries, Annals of Math. (to appear) arXiv:math.GT/0310164

[15] R Lipshitz, A cylindrical reformulation of Heegaard Floer homology arXiv: math.SG/0502404

[16] D McDuff, Singularities and positivity of intersections of J-holomorphic curves, from: "Holomorphic curves in symplectic geometry", Progr. Math. 117, Birkhäuser, Basel (1994) 191-215 MR1274930

[17] P Ozsváth, Z Szabó, Absolutely graded Floer homologies and intersection forms for four-manifolds with boundary, Adv. Math. 173 (2003) 179-261 MR1957829

[18] P Ozsváth, Z Szabó, Holomorphic disks and topological invariants for closed threemanifolds, Ann. of Math. (2) 159 (2004) 1027-1158 MR2113019

[19] P Ozsváth, Z Szabó, Heegaard Floer homology and contact structures, Duke Math. J. 129 (2005) 39-61 MR2153455

[20] B Parker, Holomorphic curves in Lagrangian torus fibrations, PhD thesis, Stanford University (2005)

[21] M Schwarz, Cohomology operations from $S^{1}$ cobordisms in Floer homology, $\mathrm{PhD}$ thesis, ETH Zürich (1995)

[22] R Siefring, Intersection theory of finite energy surfaces, PhD thesis, New York University (2005)

[23] C H Taubes, Pseudoholomorphic punctured spheres in $\mathbb{R} \times\left(S^{1} \times S^{2}\right)$ : properties and existence, (preprint)

[24] C H Taubes, The geometry of the Seiberg-Witten invariants, Doc. Math. (1998) 493504 MR1648099

[25] C H Taubes, Seiberg Witten and Gromov invariants for symplectic 4-manifolds, First International Press Lecture Series 2, International Press, Somerville, MA (2000) MR1798809

[26] C H Taubes, Seiberg-Witten invariants, self-dual harmonic 2-forms and the HoferWysocki-Zehnder formalism, from: "Surveys in differential geometry", Surv. Differ. Geom., VII, Int. Press, Somerville, MA (2000) 625-672 MR1919438

[27] C H Taubes, A compendium of pseudoholomorphic beasts in $\mathbb{R} \times\left(S^{1} \times S^{2}\right)$, Geom. Topol. 6 (2002) 657-814 MR1943381 
[28] V Turaev, Torsion invariants of $\mathrm{Spin}^{c}$-structures on 3-manifolds, Math. Res. Lett. 4 (1997) 679-695 MR1484699

[29] C Wendl, Finite energy foliations and surgery on transverse links, PhD thesis, New York University (2005)

Department of Mathematics, University of California

Berkeley CA 94720, USA

Department of Mathematics and Statistics, University of Massachusetts

Amhurst MA 01003-9305, USA

hutching@math.berkeley.edu, sullivan@math.umass.edu

http://math.berkeley.edu/ hutching/, http://www.math.umass.edu/

sullivan/

Proposed: Robion Kirby

Received: 5 October 2004

Seconded: Peter Ozsváth, Tomasz Mrowka

Revised: 27 December 2005 